A Dissertation
presented to
the Faculty of the Graduate School
at the University of Missouri-Columbia

In Partial Fulfillment

of the Requirements for the Degree

Doctor of Philosophy

by

YUEXU JIANG

Dr. Dong Xu, Advisor

July 2021 
The undersigned, appointed by the dean of the Graduate School, have examined the dissertation entitled

\section{AN INTERPRETABLE PROTEIN LOCALIZATION PREDICTION FRAMEWORK}

presented by Yuexu Jiang, candidate for the degree of Doctor of Philosophy and hereby certify that, in their opinion, it is worthy of acceptance.

[Dr. Dong Xu]

[Dr. Jianlin Cheng]

[Dr. Toni Kazic]

[Dr. Trupti Joshi] 


\section{ACKNOWLEDGEMENTS}

Many people to thank at this moment, I want to start with my family. They allow me to choose the career I love which is a high-risk and never-ending journey to most people. Fortunately, my parents and wife could see the value in it. They believe in education and believe in me. They support me with all their force all the time and never give me too much stress. The breakout of COVID-19 made my parents stuck in the US. This is a difficult time for them since they have nowhere to go and could not speak English. They just stay at home and take care of our life. Especially after my wife gave birth to our twin boys, life would be a mess and it would be impossible to finish my Ph.D. without help from my parents. I love and own my family too much.

I would like to thank my advisor, Dr. Dong Xu. I started working in his Digital Biology Lab (DBL) as a visiting scholar. During that year, I admired his enthusiastic and rigorous attitude in research. That is when I made up my mind to continue my study in DBL even it means several years more to graduate. Dr. Dong Xu sees problems from both the whole picture and the technique details. So he can always give me many good suggestions during my Ph.D. program. I still remember he once talked to me that the study of Ph.D. should be like a "T" which has a broad range of knowledge but master one specific area deeply. He gave me a lot of freedom to try different projects, including bioinformatics data analysis, webserver/tool development, and deep learning algorithm design. That is how I gradually find what I am interested in.

I would like to express my gratitude to my committee members, Dr. Jianlin Cheng, Dr, Toni Kazic, and Dr. Trupti Joshi. I took many classes of them and learned a lot of 
machine learning and biology knowledge and skills. I also appreciate all the scientific guidance and suggestions they gave me during my qualifying and comprehensive exams. I have several collaborations with Dr. Trupti Joshi, who shows great leadership and efficiency in work. I also appreciate all the external collaboration opportunities she provides to me.

Finally, I want to thank all the people in the DBL group. It was pleasant to do research and have activities with them. Especially thank Dr. Juexin Wang, who helped me set up when I first arrived in the US and managed the lab very well to make my academic life easier. 


\section{Contents}

ACKNOWLEDGEMENTS ....................................................................................... ii

LIST OF TABLES ............................................................................................... vii

LIST OF FIGURES .............................................................................................. viii

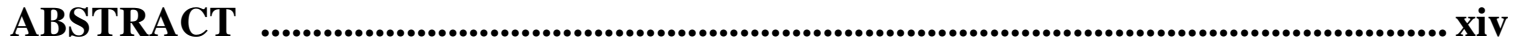

CHAPTER 1.INTRODUCTION.............................................................................................. 1

CHAPTER 2.LITERATURE REVIEW ............................................................................. 7

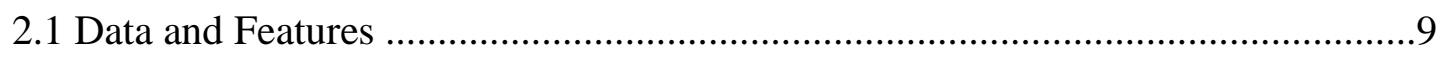

2.1.1 Sequence-based features ..........................................................................

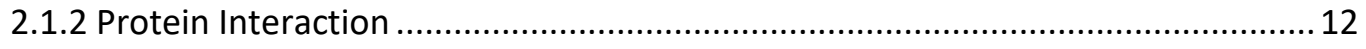

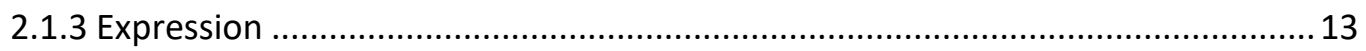

2.2 Classification Algorithms .............................................................................13

2.2.1 SVM

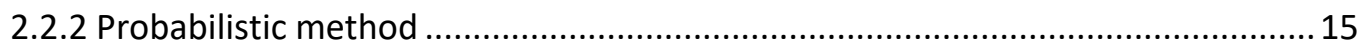

2.2.3 Distance-based methods................................................................................... 18

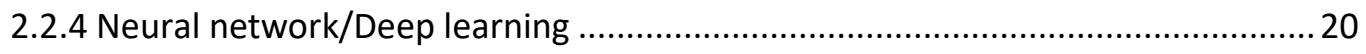

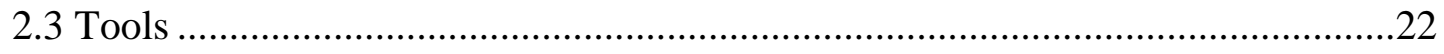

CHAPTER 3.MULOCDEEP FRAMEWORK AND WORKFLOW.......................... 25

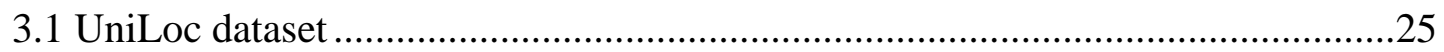

3.2 MULocDeep framework and the workflow ……………………………….....28

3.2.1 Related methods used in MULocDeep framework .............................................29

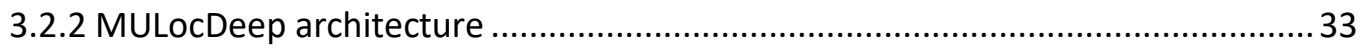

3.2.3 Parameter tuning and neural network training .............................................. 36 
3.2.4 Variant model.

\section{CHAPTER 4.PERFORMANCE EVALUATION AND MECHANISM}

INTERPRETATION ............................................................................. 43

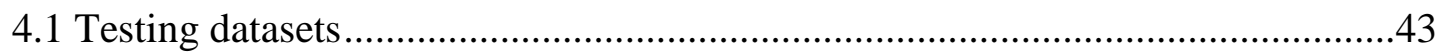

4.1.1 The three-species mitochondrial proteome dataset (Mito3) . ............................. 43

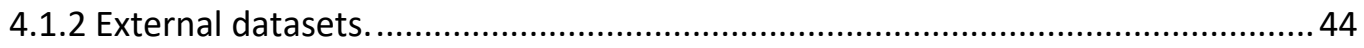

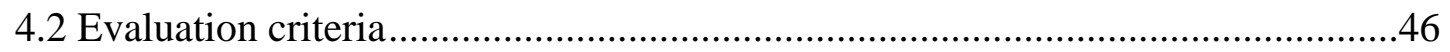

4.3 Comparison of performance in localization prediction ......................................4

4.4 Attention weight visualization ..........................................................................54

4.5 Protein localization mechanism interpretation powered by attention....................55

CHAPTER 5.MULOCDEEP WEBSERVER .................................................................... 66

5.1 MULocDeep webserver architecture .............................................................66

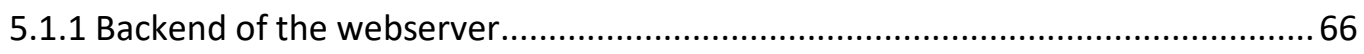

5.1.2 Frontend interface of the webserver ..................................................................

5.2 Application of MULocDeep webserver to the human proteome...........................69

CHAPTER 6.DISCUSSION AND CONCLUSION .................................................. 71

6.1 Discussion ....................................................................................................

6.1.1 Noticed issues in the MULocDeep project .................................................... 71

6.1.2 Other types of protein localization mechanisms ............................................ 72

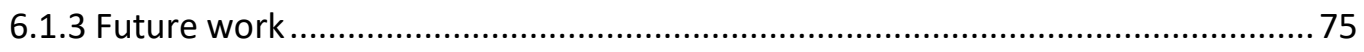

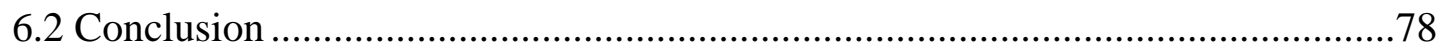

APPENDIX A. ATTENTION WEIGHTS OF PROTEINS AT THE

SUBORGANELLAR LOCALIZATIONS ............................................. 82 
APPENDIX B. GLAM2 ANALYSIS ON HIGH ATTENTION WEIGHT FRAGMENTS FROM SUBCELLULAR LOCALIZATION ............. 92

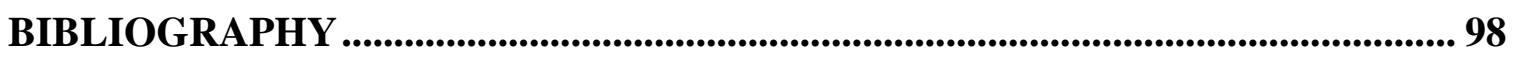

VITA 


\section{LIST OF TABLES}

Table 2.1. Summary of protein sub-cellular localization prediction tools....................... 23

Table 2.2. Summary of protein sub-organellar localization prediction tools ................... 24

Table 3.1. The statistics of samples in each localization in the UniLoc dataset.............. 27

Table 3.2. Cross-Validation MCC at the Suborganelle Level with Different Thresholds

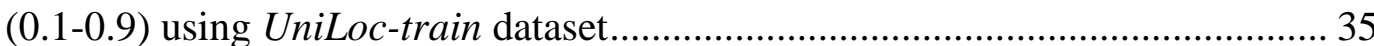

Table 3.3. Searching Range and Optimized Values of the Hyperparameters Through the

Bayesian Optimization Process.................................................................. 38

Table 3.4. Performance of Ensemble Models on Different Hyperparameter

Configurations.... 39

Table 3.5. Performance of Single Models on Different Hyperparameter Configurations 40

Table 4.1. Statistics from the Datasets Used in the DeepMito Method ........................... 46

Table 4.2. Number of proteins in each localization class in the DeepLoc dataset........... 46

Table 4.3. Evaluation and Comparison of Protein Localization Prediction Methods. ..... 48

Table 4.4. Comparison of Multi-label Protein Localization Prediction Performance...... 51

Table 4.5. Comparison of Method Effectiveness between MULocDeep and DeepMito. 52

Table 4.6. Comparison of Method Effectiveness between MULocDeep and DeepLoc... 53 


\section{LIST OF FIGURES}

Figure 2.1. Relationship among the data, features, models, and prediction outputs in computational prediction of protein localization. The sequence data can be converted into different features before feeding into a classifier model. Some classification models take raw data (e.g., one-hot-encoding of protein sequence for deep learning) as input, while others use engineered features. The localization prediction (at sub-cellular and/or suborganellar level) is the most common output. Some methods also provide side product predictions such as target peptides, signal peptide cleavage sites, and mechanism interpretability at an amino-acidlevel resolution (AAI). Homology-based method is special in the sense that it can make prediction directly based on homology-based features, such as the GO terms

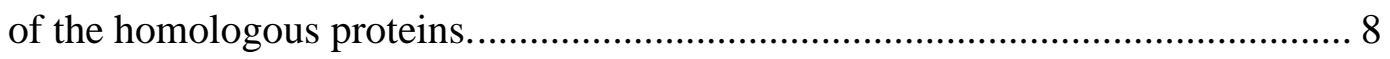

Figure 3.1. MULocDeep workflow and neural network architecture. The workflow is composed of four steps: (1) Protein sequence representation, (2) the MuLocDeep model training, (3) localization signal visualization, and finally (4) localization prediction. The details of the neural network architecture are displayed in the right

panel. 28

Figure 3.2. Illustration of LSTM architecture. The forget gate, input gate, and output gate are marked with different colors. 30

Figure 3.3. Bayesian optimization process. The plot on the left shows the distance between consecutive samplings. The plot on the right shows the ACC increase (decrease after multiple -1) along with the iteration number. 
Figure 3.4. Deep learning architecture of the variant model. The cell hierarchical matrix is replaced by a fully connected layer with the size equal to the number of classes to predict.

Figure 4.1. Visualization of attention weights for (A) SV40 large T antigen (P03070), (B) lactalbumin (P09462), and (C) cytochrome oxidase subunit 4 (P04037). The xaxis presents the sequence position from the $\mathrm{N}$-terminus to the $\mathrm{C}$-terminus, and the $y$-axis presents the value of attention weights. The region of the known sorting motif is highlighted in peach and labelled with the sequence of known localization signals. 56

Figure 4.2. The attention weights at the N-terminus (left column) and the C-terminus (right column) for proteins localized in the extracellular, mitochondrion, plastid, and thylakoid lumen

Figure 4.3. The attention weights at the N-terminus (left column) and the C-terminus (right column) for proteins localized at the nucleus, cytoplasm, and cell membrane. 58

Figure 4.4. The attention weights at the N-terminus (left column) and the C-terminus (right column) for proteins localized at the endoplasmic reticulum, Golgi apparatus, lysosome, and peroxisome 59

Figure 4.5. The attention weight vs. sequence position by aligned $\mathrm{N}$-terminal sequences at the cleavage site for proteins localized at extracellular, mitochondrion, plastid and thylakoid lumen. The vertical lines indicate the cleavage sites. For extracellular proteins, the range covers 50 AAs before the cleavage cite and 100 AAs after the cleavage cite. For the other three classes of proteins, the range 
covers 100 AAs before the cleavage cite and 50 AAs after the cleavage cite. The number of protein sequences in each class is shown. 60

Figure 4.6. The attention weights at the $\mathrm{N}$-terminus (left column) and the C-terminus (right column) for proteins of randomly shuffled sequences in the nucleus, cytoplasm, extracellular, and mitochondrion

Figure 4.7. The ratios of $\mathrm{N}$-terminal attention weights from real protein sequences to $\mathrm{N}$ terminal attention weights from randomly shuffled protein sequences in the same protein class. 62

Figure 4.8. Attention weights of 50 amino acids vs. sequence position near the termini at the sub-organellar level, which suggests potential localization signals. (A) Nterminus of cytoplasmic granule. (B) N-terminus of cell surface. (C) N-terminus of endoplasmic reticulum lumen. (D) N-terminus of Golgi apparatus and Golgi stack membrane. (E) C-terminus of endoplasmic reticulum lumen. (F) C-terminus of peroxisome proteins. 63

Figure 4.9. The top three GLAM2 results for segments from nucleus proteins. For each result, its rank, score, sequence logo and the regular expression (RE) of the motif are given 65

Figure 5.1. The architecture of the MULocDeep webserver. 67

Figure 5.2. MULocDeep webserver screenshots. (A) job submission page. (B) job management page. (C) result presentation page. (D) webserver usage distribution and statistics. 68

Figure 5.3. The human proteome localization pie chart. The left pie chart is based on the protein localization annotation, including with or without annotation, and with or 
without experimental evidence (ECO: 0000269). The green part in the left pie chart are proteins with experimentally verified localization annotations (ECO: 0000269). "Others" include those proteins contain annotations not in any of the 10 major sub-cellular or 44 sub-organellar localization annotations in MULocDeep, or have different localizations for different protein isoforms. The right pie chart is based on the distribution of prediction for the proteins in the orange part in the left pie chart.

Figure A.1. The attention weights at the N-terminus (left column) and the C-terminus (right column) for protein localizations in the nucleus organelle. 82

Figure A.2. The attention weights at the $\mathrm{N}$-terminus (left column) and the $\mathrm{C}$-terminus (right column) for protein localizations under the nucleus organelle (continue).. 83

Figure A.3. The attention weights at the N-terminus (left column) and the C-terminus (right column) for protein localizations under the cytoplasm category.

Figure A.4. The attention weights at the $\mathrm{N}$-terminus (left column) and the $\mathrm{C}$-terminus (right column) for protein localizations under the cytoplasm category.

Figure A.5. The attention weights at the N-terminus (left column) and the C-terminus (right column) for protein localizations under the membrane category.

Figure A.6. The attention weights at the N-terminus (left column) and the C-terminus (right column) for protein localizations under the membrane category.

Figure A.7. The attention weights at the $\mathrm{N}$-terminus (left column) and the C-terminus (right column) for protein localizations under the endoplasmic reticulum organelle. 88 
Figure A.8. The attention weights at the $\mathrm{N}$-terminus (left column) and the $\mathrm{C}$-terminus (right column) for protein localizations under the Golgi apparatus organelle...... 89

Figure A.9. The attention weights at the N-terminus (left column) and the C-terminus (right column) for protein localizations under the lysosome/vacuole organelle... 90

Figure A.10. The attention weights at the N-terminus (left column) and the C-terminus (right column) for protein localizations under the peroxisome organelle. 91

Figure B.1. The top 3 GLAM2 results for proteins in the cytoplasm. For each result, its rank, score, sequence logo, and the regular expression (RE) of the motif are given.

Figure B.2. The top 3 GLAM2 results for proteins in the cell membrane. For each result, its rank, score, sequence logo, and the regular expression (RE) of the motif are

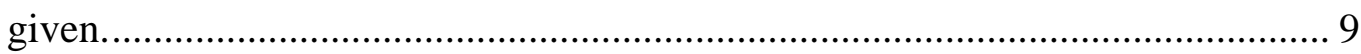

Figure B.3. The top 3 GLAM2 results for proteins in the endoplasmic reticulum. For each result, its rank, score, sequence logo, and the regular expression (RE) of the motif are given. 94

Figure B.4. The top 3 GLAM2 results for proteins in the Golgi apparatus. For each result, its rank, score, sequence logo, and the regular expression (RE) of the motif are given. 95

Figure B.5. The top 3 GLAM2 results for proteins in the lysosome. For each result, its rank, score, sequence logo, and the regular expression (RE) of the motif are given. 
Figure B.6. The top 3 GLAM2 results for proteins in the peroxisome. For each result, its rank, score, sequence logo, and the regular expression (RE) of the motif are given. 


\begin{abstract}
Protein localization is related to many human diseases. Therefore, the prediction of protein localization is an essential task that has been extensively studied. Additionally, the study of the localization mechanism can provide more biological insights and testable hypotheses. In this thesis, we propose MULocDeep, a general deep learning-based localization prediction framework. We designed a matrix layer in its architecture to reflect the hierarchical relationships of localization in cells. This enables MULocDeep, to predict multiple localizations of a protein at both subcellular and suborganellar levels. We collected a dataset with 44 suborganellar localization annotations in 10 major subcellular compartments - the most comprehensive suborganelle localization dataset to date. Our collaborators also experimentally generated an independent dataset of mitochondrial proteins in Arabidopsis thaliana cell cultures, Solanum tuberosum tubers, and Vicia faba roots and made this dataset publicly available. Evaluations using the above datasets show that overall, MULocDeep outperforms other major methods at both subcellular and suborganellar levels. We also applied Long short-term memory (LSTM) and the multi-head self-attention in MULocDeep to pursue a single amino acid level resolution when assessing their contributions to localization. This provides insights into the mechanism of protein sorting and localization motifs. Many of the candidate sites or motifs match the existing localization knowledge. A web server can be accessed at https://www.mu-loc.org/.
\end{abstract}




\section{CHAPTER 1. INTRODUCTION}

Eukaryotic cells contain well-organized compartments with different protein constituents. Although most proteins are synthesized in the cytosol, about half of them have to be transported into or across at least one cellular membrane to reach their functional destination [1-3]. Aberrant localization of proteins often has harmful effects, including diseases in human and animals [4-6], and poor traits in plants [7]. Hence, identifying protein localization and studying the mechanism of protein localization is important in a broad range of applications, such as plant breeding, pathological analysis, and therapeutic modification of the disease-related protein mislocalization $[5,8]$.

The major components of the eukaryotic cell are cytosol, nucleus, nucleolus, endoplasmic reticulum (ER), Golgi complex, mitochondria, and peroxisome (and plastids for plants). Whereas gene transcription takes place within the nucleus, protein synthesis is confined to the cytosol and takes place either on free RNA ribosomes or on ribosomes associated with the ER. There are two major mechanisms, namely co-translational and post-translational translocation $[1,2,9]$ for protein localization. Most proteins destined to be secreted from the cell, or to reside in the plasma membrane, the lysosomes, the Golgi apparatus or the ER, follow the secretory pathway and enter the ER before the end of translation, a process known as co-translational translocation [2]. Proteins targeted to the mitochondria, peroxisome and nucleus are translocated after their synthesis is complete, a process known as post-translational translocation [2, 10-13].

Protein localization is also a complex biological process which is controlled/affected by many factors, including signal peptides, protein trafficking, protein-protein 
interaction, folding, and alternative splicing, etc. [5, 14]. Among them, protein localization guided by targeting peptides is the most common mechanism [15], which includes pre-sequences and internal signals $[10,16]$. In early years it was postulated that a processing difference between free and ER-bound ribosomes led to localization. But in 1970s, Blobel hypothesized that protein targeting relied on characteristics inherent to the proteins, rather than a difference in ribosomes. Supporting his hypothesis, Blobel discovered that many proteins have a short amino acid sequence at the $\mathrm{N}$-terminal region that functions like a postal code specifying an intracellular or extracellular destination [17]. He described these short sequences (generally 13 to 36 amino acids residues) as signal peptides or signal sequences and was awarded the 1999 Nobel prize in Physiology for his findings. Signal peptide is now known as one of pre-sequence targeting signals that are found at the $\mathrm{N}$ - or $\mathrm{C}$-terminus of a protein sequence with an enrichment of charged or hydrophobic amino acids. The other types of pre-sequence targeting signals can be found in mitochondrial proteins, plastid proteins, and peroxisome proteins. Besides pre-sequence targeting signals, there is internal signals in the middle of sequences. One example is nuclear localization signal (NLS) that directs proteins to the nucleus and is often a unit consisting of five basic, positively charged amino acids. The NLS normally is located anywhere on the peptide chain.

Although the knowledge of localization mechanism is accumulating, the process by which the precursor proteins are directed to the target organelle is only partially understood [16] and only a small number of targeting peptides (especially internal signals) have been experimentally identified. According to the annotation in UniProt (release 2020_05), out of the reviewed 20,394 human proteins, 7348 have localization 
annotation with experimental verification, while only $3608(17.7 \%)$ proteins have known targeting peptides. Furthermore, very limited sub-organellar compartment localization data are available. According to our recent research, out of the 16,213 human proteins in the 10 organelles on which we focused, 5882 have experimentally verified organellar localization annotation, while only 3518 have experimentally verified sub-organellar localization annotation. Targeting peptide and sub-organelle data for non-human species are much scarcer.

Protein localization could be analyzed by several experimental approaches. Quantitative mass spectrometric read-outs allow identification of proteins across fractions [18-21]. Spatially and temporally resolved proteomic maps in living cells can be obtained by targetable peroxidase [22-24], such as APEX (Enhanced Ascorbate peroxidase) [25]. Techniques such as immunofluorescence and high-resolution confocal microscopy have enabled researchers to visually estimate localization of a protein within a single living cell [26-30]. One problem of experimental methods is that the throughput is low and the cost is high. In addition, results from different experimental techniques are not always consistent [29]. For example, Thul et al. classified 12,003 proteins of 22 human cell lines into 13 major organelles based on more than 80,000 immunofluorescence microscopy (IF) images in the Human Protein Atlas (HPA). However, the overall agreement by mass spectrometry validation was only $46 \%$ [29]. Thus, computational methods can play an important role in this area. Computational protein localization identification can be complementary to experimental approaches. Experimental annotations are usually used as true labels for computational methods to predict the localization of other proteins. Due to their automated and high-throughput nature, computational methods are attractive for the 
large-scale assignment of protein subcellular locations. Furthermore, the availability of various types of data can help computational methods for protein localization prediction.

So far, many computational methods have been proposed, some of them have achieved good prediction results on specific protein localization cases. However, these methods still face limitations and many unsolved problems. Suborganellar localizations are only studied by some organelle-specific predictors. Most of the methods focus on the prediction of protein localization at the subcellular level. A systematic suborganelle localization prediction tool at the whole-cell scale is still missing. Furthermore, protein localization is a multi-label problem, i.e., one protein may be found in several different compartments in a cell. Some methods neglected this fact for model simplicity and better performance. In addition, there is room to increase the deep learning model's interpretability for characterizing localization signals. One direction is to increase the detection coverage, i.e., identify the targeting peptides in the middle of protein sequence rather than just the N-termini where most of the known targeting peptides locate. Another potential improvement is the interpretation resolution. A single amino acid level resolution would gain more insight regarding localization mechanism. However, some recent interpretable models utilized convolutional neural network (CNN) which would decrease the resolution.

In this thesis, we introduce a multi-label protein localization framework named MULocDeep that covers 10 main subcellular localizations and 44 suborganellar localizations. A matrix layer is designed to capture the intrinsic hierarchical relationships between organelles and their subcompartments, enabling our method to make predictions at both levels simultaneously. The MULocDeep framework uses the Long Short Term 
Memory (LSTM) [31] and multi-head self-attention[32], which have the ability to extract biological features that contribute to localizations at the single amino acid resolution. Some of these features match the current knowledge of protein sorting signals, while there are novel discoveries that could provide some new insights. This work also includes an experimental study, in which the mitochondrial proteomes of 3 species, including Arabidopsis cell cultures, Solanum tubers, and Vicia roots, were extracted and identified (Mito3 dataset). We also systematically collected a dataset from the UniProt database[33], containing proteins of eukaryotic species in 44 suborganellar compartments in 10 subcellular localizations with experimental evidences (UniLoc dataset). Evaluations using the above datasets show that overall, MULocDeep outperforms other major methods at both subcellular and suborganellar levels. The datasets themselves can be used as benchmarks for methods developed by others. The source code and the web server of our method are publicly available.

The chapters in this thesis are organized as follows:

In Chapter 2, we make a literature review to introduce the features, algorithms, and tools that are commonly used in computational localization prediction.

In Chapter 3, we introduce the MULocDeep framework including its workflow, datasets, configuration details, and related methods.

In Chapter 4, we show the localization prediction performance of MULocDeep and compare with other methods or tools. Then we introduce the usage of attention mechanism in MULocDeep and illustrate its power in localization mechanism analysis.

In Chapter 5, we present the webserver of MULocDeep. 
In Chapter 6, we summarize the contributions of this thesis and point to directions of future work. 


\section{CHAPTER 2. LITERATURE REVIEW}

Computational prediction of protein localization provides an important approach for studying protein localization and it has been an active research area for more than two decades. In particular, recent machine learning advancement fueled new method development in this area. In this review chapter, we first categorize the main features and algorithms in protein localization prediction. Then we summarize a list of protein localization prediction tools in terms of their coverage, characteristics, and accessibilities for guiding users to suitable tools based on their needs.

Several papers already reviewed protein localization prediction methods. The review of [34] focused on the methods for bacterial protein localization prediction. Some reviews $[35,36]$ mainly covered protein sequence features (such as targeting peptides) in localization prediction. Another review mainly discussed the web-based prediction tools for human protein subcellular localization [37]. General methods and tools for protein localization prediction were introduced in review papers [38-40], which have a scope similar to the one of this review. However, the most recent one was published in 2014. Many new methods have been proposed and greatly improved the prediction accuracy since then, especially the deep-learning methods. These new methods and tools are highlighted in this review while some important previous methods are summarized. This review is organized as follows. In Sections 1 and 2, we analyze the features and classifiers that are often chosen by different methods, respectively. Many of these methods provide standalone tools and/or web services and we summarize them in Section 3. For each tool, its target compartments, side products besides localization prediction, 
and accessibility is given. The relationship among data, features, models, as well as prediction outputs is shown in Figure 2.1.

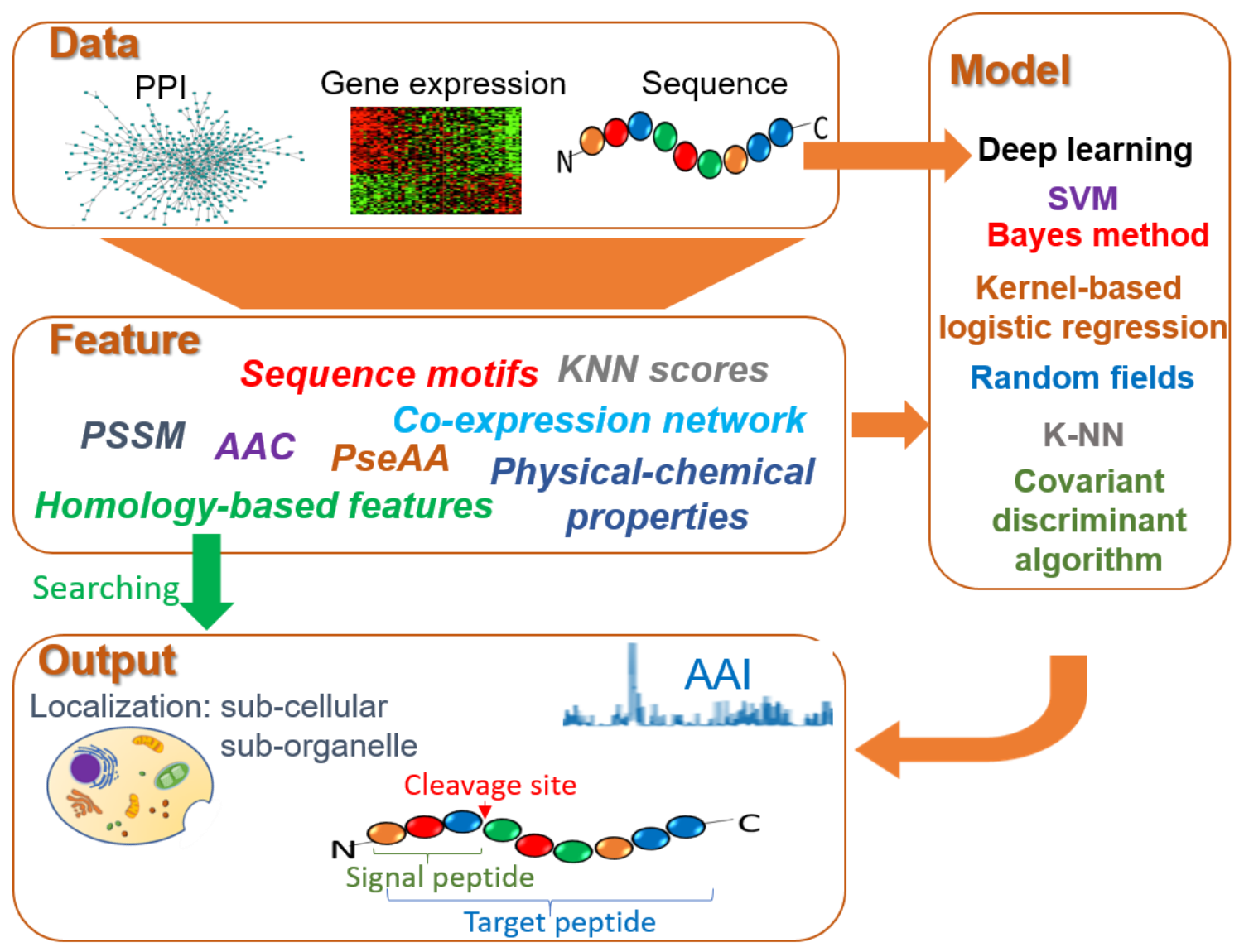

Figure 2.1. Relationship among the data, features, models, and prediction outputs in computational prediction of protein localization. The sequence data can be converted into different features before feeding into a classifier model. Some classification models take raw data (e.g., one-hot-encoding of protein sequence for deep learning) as input, while others use engineered features. The localization prediction (at sub-cellular and/or suborganellar level) is the most common output. Some methods also provide side product predictions such as target peptides, signal peptide cleavage sites, and mechanism interpretability at an amino-acid-level resolution (AAI). Homology-based method is special in the sense that it can make prediction directly based on homology-based features, such as the GO terms of the homologous proteins. 


\subsection{Data and Features}

\subsubsection{Sequence-based features}

Protein sequence is considered the most important source of information for protein localization prediction, especially the terminal regions where the targeting signals are likely to be found. Protein sequence information could be obtained from many databases such as the UniProt knowledgebase [33]. Many types of features have been proposed based on protein sequences.

\subsubsection{Amino acid composition}

Probably the simplest feature to represent protein sequence is through amino acid (AA) composition [41]. Given a protein sequence $P$, the AA composition of $P$ can be expressed by

$$
P=\left[f_{1} f_{2} \ldots f_{20}\right]^{T},
$$

where $f_{u}(u=1,2, \ldots, 20)$ are the normalized occurrence frequencies of the 20 native amino acids in protein $P$.

\subsubsection{2 pseAA composition}

The main shortcoming of the AA composition feature is lack of the protein sequence order information [39]. The concept of pseudo amino acid composition (PseAA) was proposed to address this issue [42] by representing a protein as a vector $P$ :

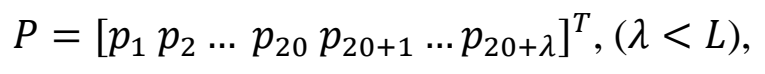

where the $20+\lambda$ components are given by 


$$
P_{u}=\left\{\begin{array}{lr}
\frac{f_{u}}{\sum_{i=1}^{20} f_{i}+w \sum_{k=1}^{\lambda} \tau_{k}}, & (1 \leq u \leq 20) \\
\frac{w \tau_{u-20}}{\sum_{i=1}^{20} f_{i}+w \sum_{k=1}^{\lambda} \tau_{k}}, & (20+1 \leq u \leq 20+\lambda)
\end{array}\right.
$$

where $\mathrm{w}$ is the weight factor that was set at 0.05 in the original paper [42], and $\tau_{k}$ is the k-th tier correlation factor, which reflects the sequence order correlation between all of the k-th most contiguous residues as formulated by

$$
\tau_{k}=\frac{1}{L-k} \sum_{i=1}^{L-k} J_{i, i+k},(K<L)
$$

As in Eq. (2), the first 20 components are associated with the conventional amino acid composition of $\mathrm{P}$, whereas the remaining components are the $\lambda$ correlation factors that reflect the first tier, second tier, and so on so forth up to the $\lambda$-th tier sequence order correlation patterns. These $\lambda$ factors incorporate the sequence order effects, and $\lambda$ is a hyperparameter (integer) to choose. The calculation of $\tau_{k}$ integrates the hydrophobicity values $\left(H_{1}\right)$, hydrophilicity values $\left(H_{2}\right)$, and side-chain masses $(M)$ for amino acids $i$ and $i+k$ as

$$
J_{i, i+k}=\frac{1}{3}\left\{\left[H_{1}\left(R_{i+k}\right)-H_{1}\left(R_{i}\right)\right]^{2}+\left[H_{2}\left(R_{i+k}\right)-H_{2}\left(R_{i}\right)\right]^{2}+\left[M\left(R_{i+k}\right)-M\left(R_{i}\right)\right]^{2}\right\}
$$

Note that Eq. (5) is just one form for deriving the correlation factors. Other information such as the physicochemical distance and the amphiphilic pattern could also be used to derive different types of PseAA composition.

\subsubsection{Homology Information}

Subcellular localization tends to be evolutionarily conserved [43], and thus homology to a protein of known localization is often a good indicator of a protein's actual localization [44]. Such information could be derived through BLAST [45] or a more sensitive search method such as HHblits [46] against a database of proteins with known localization. One important source of known localization for homologous 
information is the cellular component of Gene Ontology (GO) [47]. It has been shown useful in improving the protein localization prediction performance [48-51].

\subsubsection{Evolutionary Profiles}

Evolutionary Profiles, represented by Position-Specific Scoring Matrix (PSSM), etc., provide informative input for protein localization prediction. PSSM indicates amino acid occurrence for each position in a protein multiple sequence alignment. PSSM scores are generally shown as positive or negative values. A positive score indicates that the given amino acid substitution occurs more frequently in the alignment than expected by chance, while a negative score indicates that the substitution occurs less frequently than expected. PSSMs can be created using PSI-BLAST, which finds similar protein sequences to a query sequence, and then constructs a PSSM from the resulting alignment.

\subsubsection{Motifs}

Certain sequence patterns may correlate with a specific subcellular localization due to localization signals or functional relationships [52]. Such motif information could be retrieved from databases, such as PROSITE [53], or by data mining approaches. One special motif is the targeting peptides which are short sequences mainly present at the termini of proteins. They function like a postal code specifying an intracellular or extracellular destination [17]. Some methods predict the presence of targeting peptides as a side product besides protein localization prediction $[54,55]$, while some methods use targeting peptides as input features to predict protein localization [52].

The sequence pattern can also be extracted through a sliding window of k-mer sequence. The motif length $k$ is often set based on specific needs or biological prior knowledge. For example, TetraMito [56] used over-represented tetrapeptides (four 
continuous amino acids that are believed to encode a specific structure) as features to predict submitochondrial protein localization. A similar idea was used in sub-Golgi protein localization prediction by SubGolgi 2.0 method [57] where an SVM classifier was trained using g-gap dipeptide compositions (two amino acids with g residues between them). LOCALIZER [58] is another k-mer-based method for predicting plant and effector protein localization to chloroplasts, mitochondria, and nuclei. The motif length $k$ varies in LOCALIZER to capture the target signals on protein sequences.

\subsubsection{Physical-chemical properties}

As the name suggests, this feature uses the AA's physical and chemical properties to represent protein sequence. These properties are already calculated and stored in public databases. According to the work of Venkatarajan and Braun [59], a comprehensive list of 237 physical-chemical properties for each amino acid was compiled from the SWISSPROT [33] and dbGET [60] databases. Their study showed that the number of properties could be reduced while retaining approximately the same distribution of amino acids in the feature space. Particularly, the correlation coefficient between the original and regenerated distances is more than $99 \%$ when using the first five eigenvectors.

\subsubsection{Protein Interaction}

If two proteins interact, they are neighbors of each other in a protein-proteininteraction (PPI) network. The localizations of its neighbors in a PPI network carry some information about the localization of the un-annotated proteins. For example, if most of the neighbors of a protein have the same localization, it is likely that the protein is localized to the same location. The definition of protein interaction varies. There are 
protein interactions based on physical connections or based on genetic regulations.

Protein interaction data can be retrieved from databases, such as MINT [61], DIP [62], BioGRID [63], and STRING [64].

\subsubsection{Expression}

The rationale of using gene/protein expression as a feature is that the genes/proteins in the same compartment at the organelle or suborganelle level tend to co-express to perform related functions. The expression information can be used in a network form just like the protein interaction feature mentioned above [65]. For example, an interaction is made if the expression correlation between two genes/proteins exceeds a predefined threshold. Expression information can also be used to create features like the k-nearestneighbor (k-NN) scores in the MU-LOC method [66], or used as features by itself in SLocX method [67]. Expression data is also very common, and they can be downloaded from databases like Gene Expression Omnibus (GEO) [68] and The Cancer Genome Atlas (TCGA) [69].

\subsection{Classification Algorithms}

\subsubsection{SVM}

The support vector machine (SVM) [70] uses kernel functions to map the input vectors into a high dimensional feature space and constructs a hyperplane that maximizes the margin between different classes. SVM can handle large feature space and effectively avoid overfitting. 
The method proposed in [71] was an early SVM-based protein localization prediction approach. To deal with a multi-class classification problem, it used AA composition as a feature to train SVM classifiers in a one-versus-rest fashion. pSLIP [72] employed SVM in conjunction with multiple physicochemical properties of amino acids to predict protein subcellular localization in eukaryotes across six different locations. The density-induced Support Vector Data Description (D-SVDD) is an extension of conventional Support Vector Data Description (C-SVDD) introduced for one-class classification task inspired by SVM [73]. PLPD [74] used AA-based and motif features to modify the D-SVDD for multi-class multi-label protein localization prediction, mainly from imbalanced training datasets. A two-level SVM system to predict protein localization was designed in [75]. The first level consists of multiple SVMs using distinct AA-based features (AA composition and physical-chemical properties). The SVM at the second level makes the final prediction; SLocX [67] used SVM to predict subcellular localization of Arabidopsis proteins using gene expression and AA composition as features.

Recent SVM-based methods include CELLO2GO [76], which combined an SVMbased localization prediction method with BLAST homology search. When homologous proteins with known localizations were available, their GO terms were used as possible functional annotations for the queried protein. Otherwise, the SVM classifier would provide localization prediction. MultiP-SChlo [77] is another SVM-based method that predicts subchloroplast protein localization with multiple labels, with features including PseAAC and AA properties. MKLoc [78] is an SVM-based method for multi-label protein localization prediction. The protein sequence is represented by a 30-dimensional 
feature vector consisting of PseAAC, physical-chemical properties, motifs from PROSITE, and GO annotations. LocTree3 [48] improved over LocTree2 [79] by including information about homologs if available through a PSI-BLAST search. MitoFates [80] is a prediction method for cleavable N-terminal mitochondrial targeting signals and their cleavage sites. Besides the classical features such as AA composition, sequence profiles, and physical-chemical properties, MitoFates introduced novel sequence features including positively charged amphiphilicity and presequence motifs. An SVM classifier was trained using these features. SChloro [81] converted protein sequence into PSSM profile and Kyte-Doolittle scale (average hydrophobicity). Two layers of SVMs were designed predicting targeting signal and membrane protein information. The final output predicted six sub-chloroplastic localization by integrating the predictions from previous layers.

\subsubsection{Probabilistic method}

\subsubsection{Bayes method}

Probabilistic models, especially the Bayes method, either Bayes Optimal Classifier or Bayesian Network, make the most probable prediction for a new example. It is described using the Bayes Theorem [82] for calculating a conditional probability. It is also closely related to the Maximum a Posteriori (MAP), a probabilistic framework that finds the most probable hypothesis for a training dataset. In large real-world applications, the Bayes method usually needs to be tempered by assuming that different features are independent of each other, such as Naïve Bayes. 
PSORT-B [52] , as well as the following versions $[83,84]$ (with higher prediction coverage and refined subcategories), constructed six analytical modules based on features, including homology, motifs, and signal peptide. A query protein undergoes each of the analyses and the results are combined using a Bayesian Network to generate a final probability value for each localization site.

\subsubsection{2 kernel-based logistic regression}

When determining the probability of a protein to be localized at a specific location given a PPI network, kernel-based logistic regression (KLR) considers the localization information of all the proteins in the network. The KLR model can be formulated as follows [85]. Given a protein-protein interaction network with $\mathrm{N}$ proteins $X_{1}, \ldots, X_{N}$ and some of them have unknown localization. Let

$$
X_{[-i]}=\left(X_{1}, \ldots, X_{i-1}, X_{i+1}, \ldots, X_{N}\right),
$$

represent the protein set exclude protein $i$. Let

$$
M_{L}(i)=\sum_{j \neq i, x_{j} \text { known }} K(i, j) I\left\{x_{j}=L\right\},
$$

represent the summed distances of protein $i$ to proteins targeting to Localization $\mathrm{L}$, where $K(i, j)$ is the kernel function for calculating the distances between two proteins in the network. Then the KLR model is given by

$$
\log \frac{\operatorname{Pr}\left(X_{i}=L \mid X_{[-i]}, \theta\right)}{1-\operatorname{Pr}\left(X_{i}=L \mid X_{[-i]}, \theta\right)}=\gamma+\delta M_{! L}(i)+\eta M_{L}(i),
$$

which means that the logit of $\operatorname{Pr}\left(X_{i}=L \mid X_{[-i]}, \theta\right)$, the probability of protein $i$ targeting a location $L$ is linear based on the summed distances of proteins targeting to $L$ or other location. Then we have

$$
\operatorname{Pr}\left(X_{i}=L \mid X_{[-i]}, \theta\right)=\frac{1}{1+e^{-\left(\gamma+\delta M_{! L}(i)+\eta M_{L}(i)\right)}},
$$


Note that the probability of being in each localization will be calculated separately as a binary classification problem.

NetLoc [65] applied KLR on protein networks constructed based on different relationships including physical PPI, genetic PPI, and coexpression. They found that networks with high connectivity and a high percentage of interacting protein pairs targeting the same location led to better prediction performance.

\subsubsection{Random Fields}

Given a probability space, a random field $T(x)$ defined in $\mathbb{R}^{n}$ is a function such that for every fixed $x \in \mathbb{R}^{n}, T(x)$ is a random variable on the probability space [86]. Among various random fields, Markov random field (MRF) and conditional random field (CRF) have been used in the protein localization prediction $[54,87]$. An MRF with respect to a graph $\mathrm{G}$ is a set of random variables corresponding to the nodes in $\mathrm{G}$ (random field) and with a joint distribution that is Markov with respect to G. In other words, the joint probability distribution associated with this MRF is subject to the Markov constraint given by G: for any two variables, $V_{i}$ and $V_{j}$, the value of $V_{i}$ is conditionally independent of $V_{j}$ given its neighbors $B_{i}$. In this case, the joint probability distribution $P\left(\left\{V_{i}\right\}\right)$ factorizes according to $\mathrm{G}$. In contrast, we can describe a CRF with respect to a graph $\mathrm{G}$ as a set of random variables corresponding to the nodes in $\mathrm{G}$, a subset $\left\{X_{i}\right\}_{i=1}^{n}$ of which are assumed to always be observed, and remaining variables $\left\{Y_{i}\right\}_{i=1}^{m}$ with a conditional distribution $P\left(\left\{Y_{i}\right\}_{i=1}^{m} \mid\left\{X_{i}\right\}_{i=1}^{n}\right)$ that is Markov with respect to G. Both MRFs and CRFs typically fit a model that can be used for conditional inference in diverse settings. The main difference is that the MRF has no consistently designated "observed variables" and needs a joint distribution over all variables that adhere to the Markov constraints of G. 
CRF was used for signal peptide cleavage site prediction in DeepSig [87] and for specific signal peptide prediction in SignalP 5.0 [54]. A tissue-specific subcellular localization prediction method was proposed in [88] using multi-label MRF. The tissuespecific network was constructed from generic physical PPI networks and tissue-specific functional associations. Tissue-specific localization annotations were obtained from HPA [89].

\subsubsection{Distance-based methods}

\subsubsection{1 k-nearest neighbors ( $k-N N)$ classification}

The K-NN algorithm is a non-parametric method used for classification and regression [90]. In both cases, the input consists of the k closest training examples in the

data set. The output depends on whether k-NN is used for classification or regression. In k-NN classification, the output is a class membership. An object is classified by a plurality vote of its neighbors, with the object being assigned to the class most common among its $\mathrm{k}$ nearest neighbors ( $\mathrm{k}$ is typically a small positive integer). If $\mathrm{k}=1$, then the object is simply assigned to the class of that single nearest neighbor.

WoLF PSORT [91] converted protein amino acid sequences into numerical localization features such as targeting signals, amino acid composition, and functional motifs. After conversion, a k-NN classifier was used for prediction; A idea similar to kNN was used in [92], where a physical interaction network was obtained from BioGRID [63], and the GO Cellular Component annotation was mapped onto the network if it is available for the corresponding protein (node). For a query protein, the percentage of its 
interactors associated with each target localization is calculated. The top two localizations are reported as the prediction.

\subsubsection{Covariant discriminant algorithm based on Mahalanobis distance}

The Mahalanobis distance [93] is a measure of the distance between a point $\mathrm{P}$ and a distribution D. It is a multi-dimensional generalization of the idea of measuring how many standard deviations away $\mathrm{P}$ is from the mean of $\mathrm{D}$. This distance is zero if $\mathrm{P}$ is at the mean of $\mathrm{D}$, and grows as $\mathrm{P}$ moves away from the mean along each principal component axis. If each of these axes is re-scaled to have unit variance, then the Mahalanobis distance corresponds to standard Euclidean distance in the transformed space. The Mahalanobis distance is thus unitless and scale-invariant, and takes into account the correlations in the data set.

The Mahalanobis distance of an observation $\vec{x}=\left(x_{1}, x_{2}, x_{3}, \ldots, x_{N}\right)^{T}$ from a set of observations with mean $\vec{\mu}=\left(\mu_{1}, \mu_{2}, \mu_{3}, \ldots, \mu_{N}\right)^{T}$ and covariance matrix $S$ is defined as:

$$
D_{M}(\vec{x})=\sqrt{(\vec{x}-\vec{\mu})^{T} S^{-1}(\vec{x}-\vec{\mu})},
$$

The similarity between the standard vector $\bar{X}^{\xi}$ (normalized occurrence frequencies of the 20 AA from class $\xi$ ) and the protein $X$ is characterized by the covariant discriminant, as defined by Liu and Chou in [94]:

$$
F\left(X, \bar{X}^{\xi}\right)=D^{2}\left(X, \bar{X}^{\xi}\right)+\ln \left(\lambda_{2}^{\xi} \lambda_{3}^{\xi} \lambda_{4}^{\xi} \ldots \lambda_{20}^{\xi}\right),
$$

where the first term is the squared Mahalanobis distance, and $\lambda_{i}^{\xi}$ is the $i$-th eigenvalue of the covariance matrix $S$.

The covariant discriminant algorithm has been used in general protein localization prediction in [94], as well as in apoptosis protein localization prediction [95] and Golgi 
protein subtype prediction [96]. The features used in these methods were AA composition or Pseudo AAC.

\subsubsection{Neural network/Deep learning}

An artificial neural network (ANN) is based on a collection of connected units or nodes called artificial neurons, which loosely model the neurons in a biological brain. Each connection, like the synapses in a biological brain, can transmit a signal to other neurons. An artificial neuron receives a signal and processes it, and the output of each neuron is computed by a non-linear function of the sum of its inputs. Increased computing power from GPUs and distributed computing allow the use of larger networks, which is known as "deep learning" [97]. It becomes the hottest field in machine learning and different architectures have been proposed, such as deep neural network (DNN) [98], convolutional neural network (CNN) [97], recurrent neural network (RNN) [31, 99], and attention mechanism [100]. These deep learning methods, as well as traditional ANN, have been applied in protein localization prediction. Due to the abstract feature extraction ability in deep learning models, artificial feature engineering sometimes is not required. Raw protein sequences can be given as inputs for many deep learning localization prediction methods [101, 102].

SCLpred [101] developed a N-to-1 neural network for protein localization prediction. Their method is capable of mapping a whole sequence into fixed-length properties so that no predefined feature is needed; DeepLoc [103] applied CNN, bidirectional long short term memory (LSTM) [31], and attention mechanism for predicting localization and detecting the regions in a protein sequence that are relevant to 
the localization prediction; MU-LOC [66] provided two models (SVM and DNN) to predict mitochondrial protein in plant specifically. The features include AA composition, PSSM, and gene expression; MULocDeep [104], from the same group that developed MU-LOC, is a recently developed deep learning method, which extends the target localization coverage to 10 main subcellular compartments and their suborganellar compartments, with 44 localization classes in total. Its deep learning model consists of bidirectional LSTM and multi-head self-attention mechanism [32]. Besides protein localization prediction, it shed some light on the mechanism of localization by highlighting the regions on protein sequences that are likely to be targeting peptides; DeepMito [105] is another deep learning method for sub-mitochondrial localization prediction using CNN. The features include physical-chemical properties and PSSM besides the one-hot encoding of raw sequence.

Some methods do not predict the localization directly but predict the presence and location of targeting peptides. The localization of corresponding proteins could be inferred roughly. DeepSig [87] and SignalP 5.0 [54] are such methods that predict signal peptides and their cleavage sites using deep-learning methods. DeepSig used CNN, while SignalP 5.0 applied CNN, bidirectional LSTM, and a CRF for specific signal peptide prediction. TargetP 2.0 [55] trained a deep learning model constructed by bidirectional LSTM and multi-attention mechanism to predict N-terminal targeting signals, which direct proteins to the secretory pathways, mitochondria, and chloroplasts or other plastids. 


\subsection{Tools}

Many of the methods mentioned above provide web servers or standalone tools. Unfortunately, some of them are not accessible due to lack of maintenance. We summarize a list of available protein localization prediction tools in terms of their coverages, characteristics, and accessibilities. The subcellular localization prediction tools are shown in Table 2.1 Note that the BUSCA [106] and the SubCons [107] tools are web servers that integrate different computational tools for protein subcellular localization prediction. For some tools, e.g. DeepSig and SignalP 2.0, their localization coverage in Table 1 is marked as SP (secretory pathway). The reason is that they are signal peptide prediction tools. A signal peptide directs a protein toward the secretory pathway where the protein either inside certain organelles (the endoplasmic reticulum, Golgi or endosomes), secreted from the cell, or inserted into most cellular membranes. Thus, the specific localization of these proteins is not unique. Some tools consider the secretory pathway as a low-resolution localization. TargetP 2.0 also predicts the presence of signal peptides. Besides that, it also predicts the targeting peptide for mitochondrial proteins and plastid proteins where the unique protein localization could be inferred. The sub-organellar localization prediction tools are shown in Table 2.2. 
Table 2.1. Summary of protein sub-cellular localization prediction tools

\begin{tabular}{|c|c|c|c|c|c|c|}
\hline Tool & Coverage & \multicolumn{3}{|c|}{ Characteristics } & \multicolumn{2}{|l|}{ Accessibility } \\
\hline & & $\begin{array}{l}\text { Target } \\
\text { peptide }\end{array}$ & $\begin{array}{l}\text { Cleavag } \\
\text { e site }\end{array}$ & $\begin{array}{c}\text { Species } \\
\text { kingdom }\end{array}$ & Web server & $\begin{array}{l}\text { Stand } \\
\text { alone }\end{array}$ \\
\hline BUSCA & $1-4,7,11-14$ & $\checkmark$ & $\checkmark$ & Eu,Pro & http://busca.biocomp.unibo.it/ & \\
\hline CELLO2GO & $1-6,8-11,15$ & & & $\begin{array}{c}\text { Eu,Pro, } \\
\mathrm{V}\end{array}$ & $\begin{array}{l}\text { http://cello.life.nctu.edu.tw/cell } \\
\text { o2go/ }\end{array}$ & \\
\hline MULocDeep_tool & $1-10$ & $\checkmark$ & & $\mathrm{Eu}$ & http://mu-loc.org/ & $\checkmark$ \\
\hline DeepLoc & $1-10$ & $\checkmark$ & & $\mathrm{Eu}$ & $\begin{array}{l}\text { https://services.healthtech.dtu.d } \\
\text { k/service.php?DeepLoc-1.0 }\end{array}$ & \\
\hline TargetP 2.0 & $\mathrm{SP}, 4,7$ & $\checkmark$ & $\checkmark$ & Eu,Pro & $\begin{array}{l}\text { https://services.healthtech.dtu.d } \\
\text { k/service.php?TargetP-2.0 }\end{array}$ & \\
\hline MU-LOC & 4 & & & $\mathrm{P}$ & http://136.32.161.178/ & $\sqrt{ }$ \\
\hline LocTree3 & $1-4,6-11$ & & & Eu,Pro & $\begin{array}{l}\text { https://rostlab.org/services/loctr } \\
\text { ee3/ }\end{array}$ & \\
\hline MitoFates & 4 & $\checkmark$ & $\checkmark$ & $\mathrm{Eu}$ & $\begin{array}{c}\text { http://mitf.cbrc.jp/MitoFates/cg } \\
\text { i-bin/top.cgi }\end{array}$ & $\sqrt{ }$ \\
\hline LOCALIZER & $1,4,7$ & $\checkmark$ & $\checkmark$ & $\mathrm{P}$ & http://localizer.csiro.au/ & $\checkmark$ \\
\hline SignalP 5.0 & SP & $\checkmark$ & $\checkmark$ & Eu,Pro & $\begin{array}{l}\text { http://www.cbs.dtu.dk/services/ } \\
\text { SignalP/ }\end{array}$ & $\checkmark$ \\
\hline DeepSig & SP & $\checkmark$ & $\checkmark$ & $\mathrm{Eu}, \mathrm{Bac}$ & $\begin{array}{l}\text { https://deepsig.biocomp.unibo.i } \\
\text { t/welcome/default/index }\end{array}$ & $\checkmark$ \\
\hline PSORTb 3.0 & $2,3,14-16$ & & & $\mathrm{Bac}$ & https://www.psort.org/psortb/ & $\checkmark$ \\
\hline WoLF PSORT & $1-4,7,11$ & & & $\mathrm{Eu}$ & https://wolfpsort.hgc.jp/ & \\
\hline Predotar & $4,6,7$ & & & $\mathrm{Eu}$ & $\begin{array}{l}\text { https://urgi.versailles.inra.fr/pre } \\
\text { dotar/ }\end{array}$ & \\
\hline SubCons & $1-4,6,8-11$ & & & Hum & https://subcons.bioinfo.se/ & \\
\hline TPpred 3.0 & 4,7 & $\checkmark$ & $\checkmark$ & $\mathrm{Eu}$ & $\begin{array}{c}\text { https://tppred3.biocomp.unibo.i } \\
\text { t/tppred3 }\end{array}$ & $\sqrt{ }$ \\
\hline MultiLoc2 & $1-4,6-11$ & & & $\mathrm{Eu}$ & $\begin{array}{c}\text { https://abi- } \\
\text { services.informatik.uni- } \\
\text { tuebingen.de/multiloc2/webloc. } \\
\text { cgi }\end{array}$ & $\checkmark$ \\
\hline YLoc & $1-4,6-11$ & & & $\mathrm{Eu}$ & $\begin{array}{c}\text { https://abi- } \\
\text { services.informatik.uni- } \\
\text { tuebingen.de/yloc/webloc.cgi }\end{array}$ & $\checkmark$ \\
\hline
\end{tabular}

The localization coverage code: 1. nucleus; 2. cytoplasm; 3. extracellular; 4. mitochondrion; 5. cell membrane; 6. endoplasmic reticulum; 7. plastid/chloroplast; 8. Golgi apparatus; 9. lysosome/vacuole; 10. peroxisome; 11.

plasma membrane; 12. organelle membrane; 13. endomembrane system; 14. outer membrane; 15. periplasmic; 16. cell wall; SP. secretory pathway. The species kingdom code: Eu (Eukaryota, including animal, plant, and fungi); Pro (Prokaryota, including Bacteria and Archaea); V (Virues); P (Plant); Bac (Bacteria); Hum (Human). 
Table 2.2. Summary of protein sub-organellar localization prediction tools

\begin{tabular}{|c|c|c|c|}
\hline \multirow[t]{2}{*}{ Tool } & \multirow{2}{*}{$\begin{array}{l}\text { Organelle (\#Sub- } \\
\text { organellar classes) }\end{array}$} & \multicolumn{2}{|l|}{ Accessibility } \\
\hline & & Web server & Standalone \\
\hline DeepMito & Mitochondrion (4) & http://busca.biocomp.unibo.it/deepmito/ & $\checkmark$ \\
\hline SubGolgi v2 & $\begin{array}{c}\text { Golgi apparatus } \\
\text { (2) }\end{array}$ & http://lin-group.cn/server/subGolgi2 & \\
\hline TetraMito & Mitochondrion (3) & http://lin-group.cn/server/TetraMito & \\
\hline Schloro & Chloroplast (6) & https://schloro.biocomp.unibo.it/welcome/default/index & $\checkmark$ \\
\hline MULocDeep_tool & $\begin{array}{c}\text { Nucleus (8) } \\
\text { Cytoplasm (8) } \\
\text { Extracellular (1) } \\
\text { Mitochondrion (5) } \\
\text { Membrane (6) } \\
\text { Endoplasmic (5) } \\
\text { Plastid (5) } \\
\text { Golgi apparatus } \\
\text { (4) } \\
\text { Lysosome (1) } \\
\text { Peroxisome (1) }\end{array}$ & http://mu-loc.org/ & $\checkmark$ \\
\hline
\end{tabular}




\section{CHAPTER 3. MULOCDEEP FRAMEWORK AND WORKFLOW}

In this chapter, we first introduce the dataset used for training and evaluating the MULocDeep framework. Then we present the MULocDeep architecture and its workflow. Some detailed strategies for sequence representation and hyperparameter tuning will be also explained.

\subsection{UniLoc dataset}

The protein sequence and localization annotations were downloaded from the UniProt database release 2020_04 [33] with the following constraints: 1. The existence code must be protein or transcript level. 2. Proteins must be complete and fragment proteins were removed. 3. Proteins that are encoded in mitochondrion, chloroplast, and plastid were removed. 4. Proteins that do not start with Methionine or have a sequence length less than 40 amino acids were removed. One protein can have more than one localization annotation, and we chose suborganelle localizations with more than 50 proteins and ignored others in this study. Finally, 44 suborganelle localizations under 10 subcellular localizations remained. This is the most comprehensive multi-label dataset for protein localization annotations down to the suborganelle level to date. For convenience, we annotate the subcellular level as lv1, and the suborganellar level as lv2. From the data we collected (UniLoc dataset), we picked the protein samples that only have subcellular localization annotations, with (lv1_exp) or without (lv1_noexp) experimental evidence 
code (ECO:0000269). The number of samples are 11,435 and 38,443, respectively. Then, from the UniLoc dataset, we picked the protein samples that have suborganellar localization annotations, with (lv2_exp, 11,204 samples) or without ECO:0000269 (lv2_noexp, 32,303 samples). The main reason why we included protein samples without ECO:0000269 is that some of the suborganellar classes have so few samples when only including proteins with ECO:0000269. For each of the 10 classes in 1v1_exp, 15\% of the protein samples were used as testing (choosing protein samples created after 2018 in UniProt first; if less than $15 \%$ of the corresponding class, then selecting randomly). Similar way was used to select the testing sample for each of the 44 suborganellar classes from lv2_exp. The testing samples at both levels construct the final testing dataset (UniLoc-test, 4532 samples), and in this way, we make sure that the localization annotations of testing samples are experimentally verified and the testing samples cover every class. Specific samples were further selected from UniLoc-test in certain cases. For example, UniLoc-sub is composed of proteins in UniLoc-test that have suborganellar localization annotations, particularly, submitochondrial proteins (176 samples), subGolgi proteins (46 samples) and subchloroplast proteins (82 samples). UniLoc-multi (687 samples) is composed of proteins in UniLoc-test that have at least two different subcellular localization annotations. UniLoc-single (3847 samples) is composed of proteins in UniLoc-test that only have one subcellular localization annotation. 
The remaining data samples (experimentally verified or not) were combine as the training dataset (UniLoc-train, 88,853). Using the testing dataset, a non-redundant training dataset (UniLoc_train_40nr) was created by removing redundant proteins in the training dataset using blastp. Specifically, we align each of the proteins (query) in the UniLoc_train against UniLoc_test. If a query protein finds a hit with sequence identity higher than $40 \%$ or E-value $10-5$, and the alignment covers more than $80 \%$ of the shorter sequence of the query and its hit, the query will be removed. The resulting UniLoc-train$40 n r$ has 33,100 proteins. The same UniLoc-test dataset was used to evaluate the model

Table 3.1. The statistics of samples in each localization in the UniLoc dataset

\begin{tabular}{|c|c|c|c|c|c|c|c|}
\hline $\begin{array}{l}\text { Subcellular } \\
\text { localization }\end{array}$ & $\begin{array}{l}\text { No. of } \\
\text { samples in } \\
\text { testing } \\
\text { dataset }\end{array}$ & $\begin{array}{l}\text { No. of samples } \\
\text { in non- } \\
\text { redundant } \\
\text { training dataset }\end{array}$ & $\begin{array}{l}\text { No. of } \\
\text { samples in } \\
\text { redundant } \\
\text { training } \\
\text { dataset }\end{array}$ & Suborganellar localization & $\begin{array}{l}\text { No. of } \\
\text { samples in } \\
\text { testing } \\
\text { dataset }\end{array}$ & $\begin{array}{l}\text { No. of samples } \\
\text { in non- } \\
\text { redundant } \\
\text { training dataset }\end{array}$ & $\begin{array}{l}\text { No. of } \\
\text { samples in } \\
\text { redundant } \\
\text { training } \\
\text { dataset }\end{array}$ \\
\hline \multirow{8}{*}{ Nucleus } & \multirow{8}{*}{1136} & \multirow{8}{*}{6650} & \multirow{8}{*}{19974} & Nucleolus & 109 & 433 & 1938 \\
\hline & & & & Nucleoplasm & 49 & 258 & 891 \\
\hline & & & & Membrane & 61 & 241 & 1134 \\
\hline & & & & Nucleus matrix & 10 & 41 & 194 \\
\hline & & & & Nucleus speckle & 38 & 241 & 764 \\
\hline & & & & PML body & 9 & 78 & 249 \\
\hline & & & & Cajal body & 6 & 25 & 125 \\
\hline & & & & Chromosome & 126 & 597 & 2703 \\
\hline \multirow{8}{*}{ Cytoplasm } & \multirow{8}{*}{984} & \multirow{8}{*}{3723} & \multirow{8}{*}{16038} & Vesicle & 105 & 677 & 2505 \\
\hline & & & & Cytoskeleton & 245 & 1490 & 5760 \\
\hline & & & & Myofibril & 19 & 139 & 318 \\
\hline & & & & Cytosol & 126 & 534 & 2418 \\
\hline & & & & Perinuclear region & 50 & 349 & 1101 \\
\hline & & & & Cell cortex & 27 & 199 & 426 \\
\hline & & & & Cytoplasmic granule & 18 & 116 & 329 \\
\hline & & & & P-body & 15 & 67 & 266 \\
\hline Sereted & 441 & 1826 & 11018 & Extracellular space & 40 & 611 & 1379 \\
\hline \multirow{5}{*}{ Mitochondrion } & \multirow{5}{*}{183} & \multirow{5}{*}{784} & \multirow{5}{*}{4193} & Inner membrane & 73 & 418 & 1883 \\
\hline & & & & Intermembrane space & 16 & 25 & 335 \\
\hline & & & & Outer membrane & 32 & 160 & 714 \\
\hline & & & & Mitochondrion matrix & 42 & 187 & 1065 \\
\hline & & & & Membrane & 21 & 107 & 438 \\
\hline \multirow{6}{*}{ Membrane } & \multirow{6}{*}{101} & \multirow{6}{*}{2645} & \multirow{6}{*}{8582} & Clathrin-coated pit & 4 & 67 & 147 \\
\hline & & & & Coated pit & 1 & 36 & 101 \\
\hline & & & & Membrane raft & 13 & 88 & 279 \\
\hline & & & & Caveola & 4 & 43 & 153 \\
\hline & & & & Cell membrane & 590 & 4839 & 13379 \\
\hline & & & & Cell surface & 11 & 103 & 235 \\
\hline \multirow{5}{*}{ Endoplasmic } & \multirow{5}{*}{62} & \multirow{5}{*}{278} & \multirow{5}{*}{981} & ER lumen & 15 & 166 & 409 \\
\hline & & & & ER membrane & 198 & 1211 & 4630 \\
\hline & & & & ER-Golgi intermediate compartment & 11 & 64 & 266 \\
\hline & & & & Microsome & 23 & 336 & 823 \\
\hline & & & & Sarcoplasmic reticulum & 6 & 57 & 163 \\
\hline \multirow{5}{*}{ Plastid } & \multirow{5}{*}{2} & \multirow{5}{*}{5} & & Amyloplast & 3 & 1 & 66 \\
\hline & & & & Chloroplast membrane & 24 & 85 & 393 \\
\hline & & & 103 & Chloroplast stroma & 27 & 43 & 334 \\
\hline & & & & Chloroplast thylakoid lumen & 6 & 9 & 59 \\
\hline & & & & Chloroplast thylakoid membrane & 29 & 41 & 680 \\
\hline & & & & Trans-Golgi network & 35 & 202 & 744 \\
\hline Golgi apparatus & 46 & 263 & 1045 & Cis-Golgi network & 11 & 44 & 248 \\
\hline & & & & Membrane & 88 & 484 & 2275 \\
\hline & & & & Golgi stack membrane & 9 & 58 & 301 \\
\hline Lysosome & 23 & 203 & 1063 & Membrane & 34 & 152 & 724 \\
\hline Peroxisome & 29 & 168 & 678 & Membrane & 13 & 90 & 269 \\
\hline
\end{tabular}


performance after removing the redundancy effect. We did not use a sequence identity threshold lower than $40 \%$, since it would lose too many samples and leave not enough samples for training in many classes. The statistics of samples in all the classes in UniLoc dataset is shown in Table 3.1.

\subsection{MULocDeep framework and the workflow}

The workflow of our framework is presented in Figure 3.1. Protein sequences with known localization information were collected, processed, and fed into our deep learning model for training. During the training process, the output of the "attention" layer was

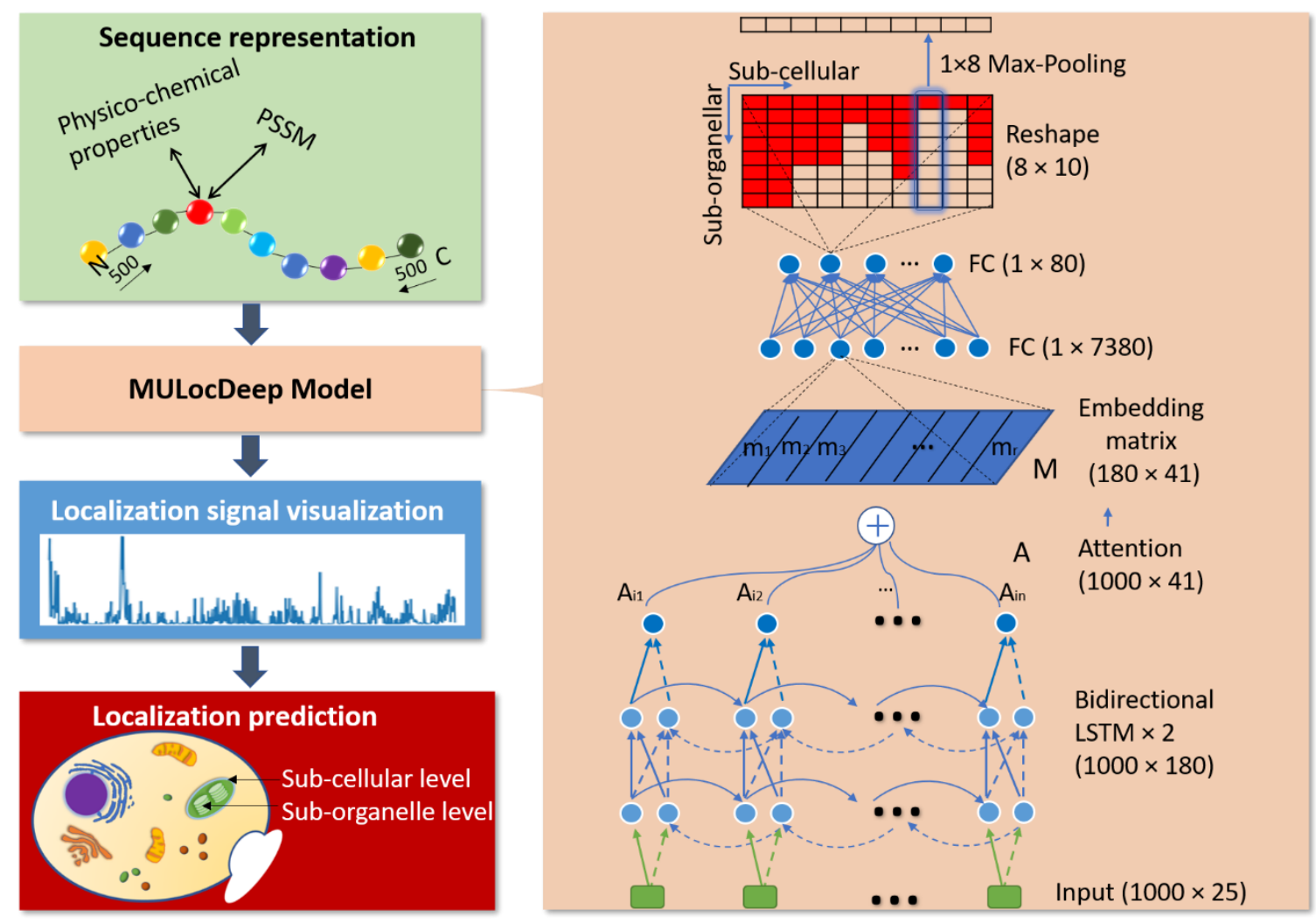

Figure 3.1. MULocDeep workflow and neural network architecture. The workflow is composed of four steps: (1) Protein sequence representation, (2) the MuLocDeep model training, (3) localization signal visualization, and finally (4) localization prediction. The details of the neural network architecture are displayed in the right panel. 
extracted separately for sorting signal interpretation and visualization. Finally, the trained model was used to predict localization for new proteins.

\subsubsection{Related methods used in MULocDeep framework}

\subsubsection{Protein sequence representation}

An encoded amino acid contains two parts. The first 5 dimensions come from the first five eigenvectors of a comprehensive list of 237 physical-chemical properties for each amino acid [59]. As we did in the domain boundary prediction study [108], these 5number descriptors can represent each amino acid for computational efficiency while maintaining almost all the information. The last 20 dimensions come from the positionspecific scoring matrix (PSSM) profile of a protein. A protein's PSSM profile is usually generated through a multiple sequence alignment against a large database. Some methods try to accelerate the process by searching a relatively small database first, and if no hit is found then use a large database instead [103, 109]. We further shortened this process by two steps, first by scanning the Swissprot [110] using PSI-blast [111]. The Swissprot database is a much smaller database than UniProt, yet most of the proteins that we studied find hits. If no hits were retrieved, in the next step, the BLOSUM62 encoding [112] was applied directly, which did not take any search time. In this way, we saved much computational time without significant performance decrease (see Results). Since the length of proteins varies, we fixed the protein-encoding length at 1000 AA. If a protein exceeded this length, the first 500 amino acids from $\mathrm{N}$-terminus and the last 500 amino acids from C-terminus were preserved and combined. If a protein had a shorter 
sequence, we padded it to $1000 \mathrm{AA}$ at the end and masked the padding part for the following calculation.

\subsubsection{Bidirectional LSTM}

The key to LSTM is the cell state C that runs through the entire chain. An LSTM unit has the ability to remove or add information to the cell state by a regulation structure called gate. Firstly, an LSTM unit uses its "forget gate" (Figure 3.2) to decide what information to discard from the cell state. It takes the output $h_{t-1}$ from the previous unit and the current input $x_{t}$ as the input of a sigmoid function to produce a number between 0 and 1 for each number in the cell state. A 1 means completely keeping the value while a 0 means completely removing it. The formulas for the forget gate is shown as Eq. (12).

$$
f_{t}=\sigma\left(W_{f} \cdot\left[h_{t-1}, x_{t}\right]+b_{f}\right)
$$

where $W_{f}$ and $b_{f}$ are the weight matrix and bias for the forget gate layer. Next, a tanh layer creates a new candidate input vector. It will be performed a pointwise product with

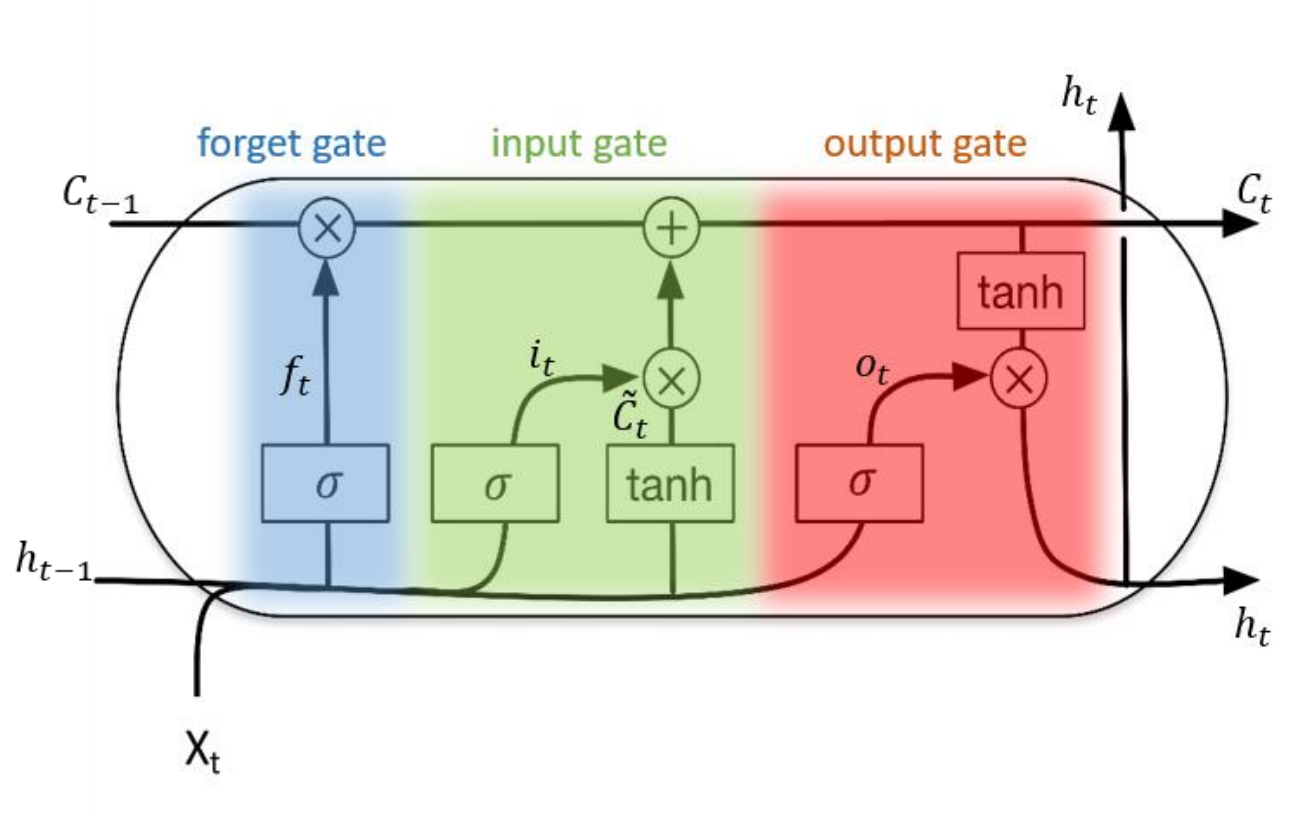

Figure 3.2. Illustration of LSTM architecture. The forget gate, input gate, and output gate are marked with different colors. 
a sigmoid layer called the "input gate" (Figure 3.2) to decide which values to add to the cell state. The formula for candidate input creation and the input gate are shown as Eq. (13) and Eq. (14), respectively.

$$
\begin{gathered}
\tilde{C}_{t}=\tanh \left(W_{C} \cdot\left[h_{t-1}, x_{t}\right]+b_{C}\right) \\
i_{t}=\sigma\left(W_{i} \cdot\left[h_{t-1}, x_{t}\right]+b_{i}\right)
\end{gathered}
$$

where $W_{C}$ and $W_{i}$ are weight matrix for the tanh layer and the input gate layer, respectively. $b_{C}$ and $b_{i}$ are bias for the tanh layer and the input gate layer, respectively. Then the LSTM unit can update the old cell state $C_{t-1}$ into the new cell state $C_{t}$ by Eq. (15).

$$
C_{t}=f_{t} * C_{t-1}+i_{t} * \tilde{C}_{t}
$$

Finally, the cell state goes through a tanh layer to scale the values between -1 and 1 . The scaled cell state will be filtered by a sigmoid layer called "output gate" (Figure 3.2) to decide which values to output. The formulas for output gate definition and the current output are shown as Eq. (16) and Eq. (17), respectively.

$$
\begin{gathered}
o_{t}=\sigma\left(W_{o}\left[h_{t-1}, x_{t}\right]+b_{o}\right) \\
h_{t}=o_{t} * \tanh \left(C_{t}\right)
\end{gathered}
$$

The ability of avoiding vanishing gradient is mainly owing to the design of forget gate in LSTM. Thus, if a protein domain boundary prediction depends on some signals from remote residues, our model can be trained to set those forget gates' values as 1 on informative positions and let the far, weak but informative signal propagate far without significant loss.

Each layer of bidirectional LSTM is composed of two LSTMs that propagate information from opposite directions. There are multiple ways to integrate the 
information from the two LSTMs, such as "sum" or "concatenate". The former will not change the embedding shape, while the latter will double the embedding dimension. In our method, we set the LSTM embedding dimension as 180 and use "sum" for integration. Thus, the tensor after the bidirectional LSTM layer is 1000 by 180 .

\subsubsection{Multi-head self-attention}

Self-attention mechanism computes the linear combination of the LSTM time steps and transform it into a fixed size embedding. The attention mechanism takes the whole LSTM hidden states $H$ (with shape of $n$ by $2 u$ ) as input, and outputs a vector of weights $a:$

$$
a=\operatorname{softmax}\left(w_{s 2} \tanh \left(W_{s 1} H^{T}\right)\right)
$$

Here $W_{s 1}$ is a weight matrix with a shape of $d a$ by $2 u$. and $w_{s 2}$ is a vector of parameters with size $d a$, where $d a$ is a hyperparameter we can set arbitrarily. Since $H$ is sized $n$ by $2 u$, the annotation vector $a$ will have a size $\mathrm{n}$. the softmax () ensures all the computed weights sum up to 1 . Then we sum up the LSTM hidden states $H$ according to the weight provided by a to get a vector representation $m$ of the input sentence.

This vector representation usually focuses on a specific component of the sentence, like a special set of related words or phrases. So it is expected to reflect an aspect, or component of the semantics in a sentence. However, there can be multiple components in a sentence that together forms the overall semantics of the whole sentence, especially for long sentences. (For example, two clauses linked together by an "and.")

The multi-head self-attention [32] uses the overall semantics of the whole sentence formed by multiple components in a sentence. So, multiple hops of attention are needed to focus on different parts of the sentence. Our method borrows this idea and sets the 
number of heads equals 41 (derived from the hyperparameter tuning). The final weight of each amino acid is the average of the 41 weights. Then we could analyse if any "important parts" of a protein sequence are responsible for the protein localization. The attention matrix A is calculated as Eq. (19)

$$
A=\operatorname{softmax}\left(W_{s 2} \tanh \left(W_{s 1} H^{T}\right)\right)
$$

where $\mathrm{H}$ is the 1000 -by-180 embedding sequence output from bidirectional LSTM. $W_{s 1}$ is a weight matrix with a shape of 369-by-180. $W_{s 2}$ is a matrix of parameters with a shape 41-by-369. The attention matrix A is returned separately for interpretation. The sequence embedding M, calculated as the weighted sum by multiplying $A$ and $H$ (Eq. 20), is also returned for further prediction.

$$
M=A H
$$

When training the models, we applied the penalization term $\mathrm{P}$ below [32]

$$
P=\left\|\left(A A^{T}-I\right)\right\|_{F}^{2}
$$

where $\mathrm{A}$ is the attention matrix, $I$ is an identity matrix, $\|\cdot\|_{F}$ stands for the Frobenius norm of a matrix. We multiple the penalization term with an attention regularizer and added the product to the model's loss. The loss gets high if two attention vectors are identical. Thus, by using this penalization term, we encourage the attention vectors to concentrate on different parts of a protein sequence.

\subsubsection{MULocDeep architecture}

A description of the MULocDeep model is shown in the right panel in Figure 3.1. The input layer was composed of encoded protein sequences with a fixed length of 1000 amino acids. Each amino acid was encoded as a 25-dimension vector (see the Methods 
section for encoding details). The input layer was followed by two layers of bidirectional LSTM [31], which ensured that every amino acid received a signal from both sides. Two such layers were stacked to give the model the ability to fit complex high-order functions. The sequence length remained unchanged after the bidirectional LSTMs, while only the encoding dimension was changed to 180 . Then a multi-head self-attention layer [32] was applied (" $\mathrm{A}$ " in Figure 1). The embedding matrix ("M" in Figure 1) was derived as the weighted sum by multiplying the attention layer with the output from the bidirectional LSTM. The attention itself was also an output to assess the contribution of each amino acid to localization. The embedding matrix was flattened into a 7380 (180X41) long vector, and then fully connected with an 80-dimensional dense layer, which was further reshaped into an 8-by-10 matrix. Each column of the matrix represents a major subcellular localization (10 organelles) and each element under the column represents a suborganelle category. In our UniLoc dataset, some organelles contained up to eight suborganellar localizations. For other organelles that had fewer suborganelle localizations, the empty slots in the matrix were padded with zeros. When processing a new sample, the predicted value in the matrix was used for the suborganelle prediction. Then a $1 \times 8$ max-pooling layer was applied to the matrix so that the highest predicted value of a suborganelle localization was used as the prediction score of the corresponding organelle localization. Only the suborganellar prediction scores were compared with the thresholds, i.e., a suborganelle with a prediction score above the threshold would trigger the prediction of corresponding organelle localization (see the Section 3.2.3 for threshold determination details). In this way, we could perform multi-label predictions at both subcellular and suborganelle levels and keep the results consistent. This matrix design 
also enables MULocDeep to utilize protein samples that only have subcellular

\section{localization annotations. The MULocDeep model was trained using the UniLoc-train}

dataset, which was divided into 8 folds. We performed cross-validation by using 7 folds

as training and 1 fold as evaluation each time. Table 3.2 shows the cross-validation

results at the suborganelle level with different prediction thresholds. When training the

Table 3.2. Cross-Validation MCC at the Suborganelle Level with Different Thresholds (0.1-0.9) using UniLoc-train dataset

\begin{tabular}{|c|c|c|c|c|c|c|c|c|c|c|}
\hline \multirow{2}{*}{$\begin{array}{l}\text { Subcellular } \\
\text { localization }\end{array}$} & \multirow[t]{2}{*}{ Suborganellar localization } & \multicolumn{9}{|c|}{ MCC vs. threshold } \\
\hline & & 0.1 & 0.2 & 0.3 & 0.4 & 0.5 & 0.6 & 0.7 & 0.8 & 0.9 \\
\hline \multirow{8}{*}{ Nucleus } & Nucleolus & 0.41 & 0.42 & 0.44 & 0.44 & 0.44 & 0.45 & 0.45 & 0.45 & 0.46 \\
\hline & Nucleoplasm & 0.23 & 0.24 & 0.25 & 0.25 & 0.25 & 0.24 & 0.23 & 0.22 & 0.2 \\
\hline & Membrane & 0.2 & 0.22 & 0.22 & 0.23 & 0.24 & 0.25 & 0.26 & 0.27 & 0.29 \\
\hline & Nucleus matrix & 0.11 & 0.12 & 0.13 & 0.13 & 0.13 & 0.11 & 0.12 & 0.13 & 0.13 \\
\hline & Nucleus speckle & 0.31 & 0.31 & 0.31 & 0.32 & 0.32 & 0.32 & 0.32 & 0.32 & 0.32 \\
\hline & PML body & 0.16 & 0.15 & 0.12 & 0.11 & 0.08 & 0.09 & 0.1 & 0.12 & 0.13 \\
\hline & Cajal body & 0.05 & 0.03 & 0.02 & 0.02 & 0.02 & 0.02 & 0.01 & 0.01 & 0 \\
\hline & Chromosome & 0.46 & 0.47 & 0.47 & 0.48 & 0.49 & 0.49 & 0.49 & 0.5 & 0.5 \\
\hline \multirow{8}{*}{ Cytoplasm } & Vesicle & 0.21 & 0.21 & 0.22 & 0.23 & 0.23 & 0.23 & 0.24 & 0.24 & 0.24 \\
\hline & Cytoskeleton & 0.5 & 0.5 & 0.51 & 0.51 & 0.52 & 0.52 & 0.52 & 0.52 & 0.53 \\
\hline & Myofibril & 0.21 & 0.24 & 0.22 & 0.2 & 0.17 & 0.15 & 0.12 & 0.1 & 0.09 \\
\hline & Cytosol & 0.29 & 0.29 & 0.29 & 0.29 & 0.29 & 0.3 & 0.3 & 0.3 & 0.3 \\
\hline & Perinuclear region & 0.12 & 0.11 & 0.11 & 0.11 & 0.11 & 0.11 & 0.11 & 0.11 & 0.09 \\
\hline & Cell cortex & 0.12 & 0.13 & 0.13 & 0.14 & 0.14 & 0.13 & 0.13 & 0.12 & 0.12 \\
\hline & Cytoplasmic granule & 0.11 & 0.11 & 0.13 & 0.11 & 0.11 & 0.13 & 0.13 & 0.14 & 0.15 \\
\hline & P-body & 0.16 & 0.17 & 0.18 & 0.19 & 0.19 & 0.18 & 0.19 & 0.18 & 0.19 \\
\hline Secreted & Extracellular space & 0.67 & 0.68 & 0.69 & 0.69 & 0.69 & 0.69 & 0.7 & 0.7 & 0.7 \\
\hline \multirow{5}{*}{$\begin{array}{l}\text { Mitochondrio } \\
\mathrm{n}\end{array}$} & Inner membrane & 0.63 & 0.63 & 0.64 & 0.64 & 0.64 & 0.64 & 0.65 & 0.65 & 0.65 \\
\hline & Intermembrane space & 0.31 & 0.34 & 0.34 & 0.36 & 0.36 & 0.37 & 0.37 & 0.36 & 0.37 \\
\hline & Outer membrane & 0.39 & 0.41 & 0.43 & 0.44 & 0.45 & 0.47 & 0.47 & 0.48 & 0.48 \\
\hline & Mitochondrion matrix & 0.55 & 0.56 & 0.57 & 0.58 & 0.58 & 0.57 & 0.57 & 0.57 & 0.58 \\
\hline & Membrane & 0.13 & 0.12 & 0.13 & 0.13 & 0.14 & 0.15 & 0.16 & 0.17 & 0.15 \\
\hline \multirow{6}{*}{ Membrane } & Clathrin-coated pit & 0.18 & 0.2 & 0.22 & 0.22 & 0.23 & 0.24 & 0.25 & 0.25 & 0.24 \\
\hline & Coated pit & 0.17 & 0.18 & 0.19 & 0.2 & 0.22 & 0.22 & 0.21 & 0.17 & 0.17 \\
\hline & Membrane raft & 0.08 & 0.06 & 0.06 & 0.04 & 0.04 & 0.04 & 0.04 & 0.02 & 0.01 \\
\hline & Caveola & 0.16 & 0.18 & 0.15 & 0.17 & 0.18 & 0.17 & 0.14 & 0.13 & 0.13 \\
\hline & Cell membrane & 0.68 & 0.68 & 0.69 & 0.69 & 0.69 & 0.69 & 0.69 & 0.69 & 0.69 \\
\hline & Cell surface & 0.1 & 0.1 & 0.1 & 0.1 & 0.1 & 0.1 & 0.1 & 0.09 & 0.08 \\
\hline \multirow{5}{*}{ Endoplasmic } & ER lumen & 0.69 & 0.71 & 0.73 & 0.72 & 0.73 & 0.73 & 0.74 & 0.74 & 0.73 \\
\hline & ER membrane & 0.6 & 0.6 & 0.61 & 0.61 & 0.61 & 0.61 & 0.61 & 0.61 & 0.61 \\
\hline & $\begin{array}{c}\text { ER-Golgi intermediate } \\
\text { compartment }\end{array}$ & 0.26 & 0.29 & 0.29 & 0.31 & 0.32 & 0.32 & 0.3 & 0.27 & 0.21 \\
\hline & Microsome & 0.47 & 0.49 & 0.5 & 0.51 & 0.51 & 0.51 & 0.51 & 0.51 & 0.48 \\
\hline & Sarcoplasmic reticulum & 0.17 & 0.21 & 0.21 & 0.23 & 0.25 & 0.24 & 0.23 & 0.23 & 0.23 \\
\hline \multirow{5}{*}{ Plastid } & Amyloplast & 0.31 & 0.34 & 0.35 & 0.37 & 0.36 & 0.31 & 0.33 & 0.34 & 0.33 \\
\hline & Chloroplast membrane & 0.44 & 0.44 & 0.45 & 0.45 & 0.45 & 0.46 & 0.47 & 0.47 & 0.48 \\
\hline & Chloroplast stroma & 0.54 & 0.54 & 0.54 & 0.55 & 0.55 & 0.54 & 0.55 & 0.54 & 0.55 \\
\hline & Chloroplast thylakoid lumen & 0.33 & 0.33 & 0.35 & 0.33 & 0.32 & 0.34 & 0.33 & 0.29 & 0.29 \\
\hline & Chloroplast thylakoid membrane & 0.54 & 0.55 & 0.56 & 0.56 & 0.56 & 0.56 & 0.56 & 0.56 & 0.56 \\
\hline \multirow{4}{*}{$\begin{array}{c}\text { Golgi } \\
\text { apparatus }\end{array}$} & Trans-Golgi network & 0.16 & 0.16 & 0.15 & 0.15 & 0.14 & 0.13 & 0.12 & 0.1 & 0.09 \\
\hline & Cis-Golgi network & 0.24 & 0.28 & 0.28 & 0.28 & 0.29 & 0.29 & 0.28 & 0.25 & 0.24 \\
\hline & Membrane & 0.5 & 0.51 & 0.51 & 0.52 & 0.52 & 0.52 & 0.51 & 0.51 & 0.51 \\
\hline & Golgi stack membrane & 0.34 & 0.34 & 0.35 & 0.36 & 0.35 & 0.37 & 0.36 & 0.38 & 0.37 \\
\hline Lysosome & Membrane & 0.28 & 0.28 & 0.28 & 0.28 & 0.28 & 0.28 & 0.27 & 0.27 & 0.27 \\
\hline Peroxisome & Membrane & 0.52 & 0.53 & 0.52 & 0.51 & 0.52 & 0.52 & 0.53 & 0.53 & 0.53 \\
\hline
\end{tabular}


MULocDeep model, we tried different strategies to tune the hyperparameters and tested their impact on the performance. The details of the training process and hyperparameter configuration are described in the Section 3.2.3.

\subsubsection{Parameter tuning and neural network training}

The hyperparameters in the models are determined through a Bayesian optimization process. These hyperparameters include the hidden dimensions, the number of heads in attention, regularizers, dropout rates, etc. Specifically, we formulate the accuracy "ACC" as the objective function and it is a function of all the hyperparameters. A Gaussian process was used as the surrogate model to approximate the objective function. We used the expected improvement (EI) as the acquisition function, which directs sampling to areas where an improvement over the current best observation is likely. The acquisition

jitter, which trades off exploitation (high objective) and exploration (high uncertainty) was set as 0.05 .

Since the optimization process would take a long time, we used training samples provided by the DeepLoc method instead of our own UniLoc-train dataset. Since DeepLoc also focuses on the same 10 subcellular localizations of eukaryotic proteins, we assume the distribution of data should be similar between the two datasets. We divided these samples into 8 folds using CD-hit [111] and the sequence identity between proteins 
in different folds was below $40 \%$. Then, an 8 -fold cross validation was performed. Each hyperparameter has a searching space (shown in Table 3.3). During the cross validation, 7 folds were used for training a sub-model under one specific hyperparameter configuration for 40 epochs. The remaining fold evaluated the accuracy in each epoch.

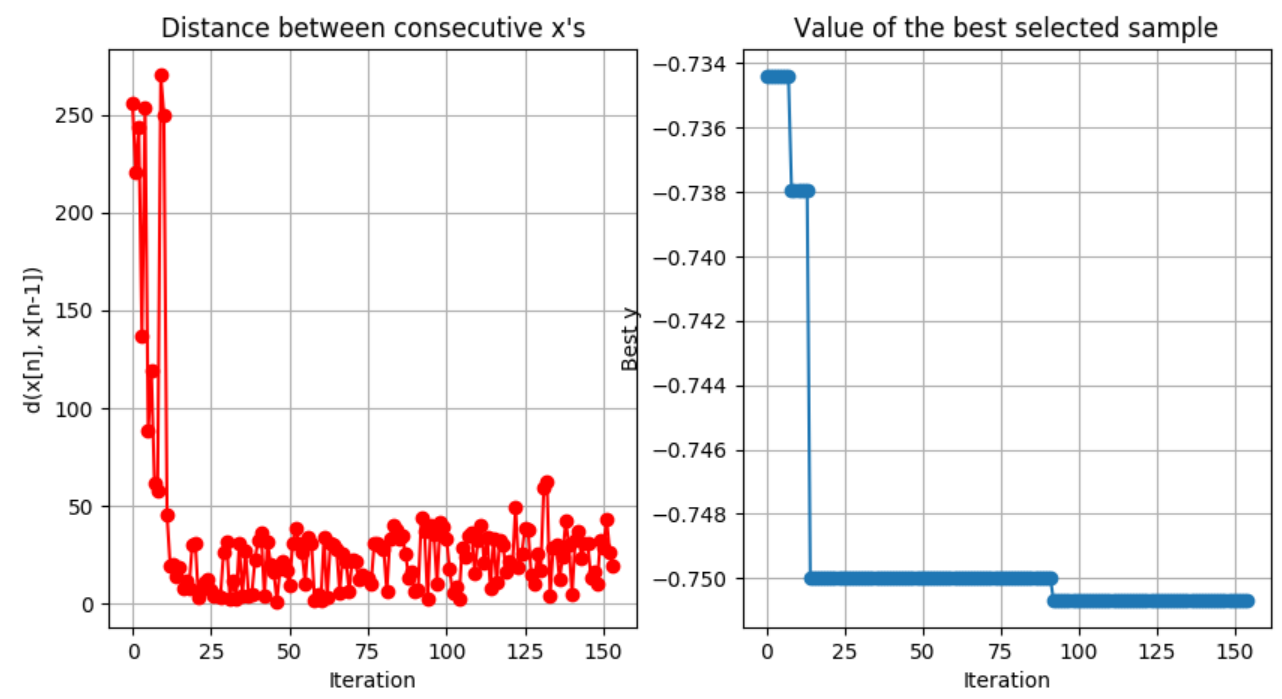

Figure 3.3. Bayesian optimization process. The plot on the left shows the distance between consecutive samplings. The plot on the right shows the ACC increase (decrease after multiple -1) along with the iteration number.

The highest accuracy on the validation fold during the 40 epochs was recorded as the accuracy for this hyperparameter configuration. In total, 150 hyperparameter combinations were tested. Figure 3.3 shows the accuracy along the testing process. The hyperparameter configuration which achieved the highest accuracy among the 150 combinations was used as the optimized configuration for this sub-model (shown in Table S6). Thus, the optimization process would run 40 (test one specific configuration) * 150 (150 configurations to test in total) $* 8$ ( 8 -fold cross-validation), which is 48,000 epochs in total. Finally, each of the eight sub-models had its own optimized hyperparameter configuration and the corresponding accuracy achieved. The 
configuration with the highest accuracy was selected as the final optimized configuration that was used for the MULocDeep model and its variant models.

The MULocDeep model is an ensemble of eight "sub-models" derived from an 8fold cross validation. Each of these eight sub-models was optimized individually and the hyperparameters of the sub-model that achieved the highest accuracy were set as the final optimum. Table 3.3 lists the hyperparameter optimization results for all eight sub-models in the cross validation. The hyperparameter configuration in sub-model 1 was selected as the final optimum. Therefore, in the MULocDeep model, all the eight sub-models used the same final optimized hyperparameters. We also tested the performance of an ensemble of sub-models where each sub-model 1) using its own optimized hyperparameters, 2) using the same, but not the final optimized, hyperparameters (select

Table 3.3. Searching Range and Optimized Values of the Hyperparameters Through the Bayesian Optimization Process

\begin{tabular}{|c|c|c|c|c|c|c|c|c|c|}
\hline \multirow[t]{2}{*}{ Parameters } & Range & \multicolumn{8}{|c|}{ Optimized Values } \\
\hline & & M1 & M2 & M3 & M4 & M5 & M6 & M7 & M8 \\
\hline $\begin{array}{l}\text { Hidden dimension } \\
\text { in LSTM }\end{array}$ & {$[32,490]$} & 180 & 220 & 389 & 303 & 264 & 392 & 230 & 273 \\
\hline $\begin{array}{l}\text { Number of heads } \\
\text { in attention }\end{array}$ & {$[16,64]$} & 41 & 27 & 47 & 37 & 38 & 38 & 57 & 26 \\
\hline $\begin{array}{l}\text { Regularizer of } \\
\text { penalization in } \\
\text { attention }\end{array}$ & $\begin{array}{c}\text { [10E-5, } \\
10 \mathrm{E}-3]\end{array}$ & $7.2 \mathrm{E}-4$ & $10 \mathrm{E}-5$ & $1.3 \mathrm{E}-4$ & $10 \mathrm{E}-5$ & 0.001 & 0.001 & $10 \mathrm{E}-5$ & 0.001 \\
\hline $\begin{array}{l}\text { Dropout rate of } \\
\text { input layer }\end{array}$ & {$[0.1,0.75]$} & 0.1 & 0.1 & 0.116 & 0.1 & 0.1 & 0.1 & 0.1 & 0.1 \\
\hline
\end{tabular}

Each sub-model (M1-M8) was optimized individually. The hyperparameters of M1, which achieved the highest prediction accuracy, were selected as the final optimum hyperparameters.

two from seven non-final optima), or 3) using the same random hyperparameters. The results are shown in Table 3.4. It turns out that the difference in performance among various ensemble models was insignificant, except for a notably poor performance by 
Table 3.4. Performance of Ensemble Models on Different Hyperparameter Configurations

\begin{tabular}{|c|c|c|c|c|c|c|}
\hline \multicolumn{2}{|c|}{ Hyperparameters that each sub-model uses } & Config 1 & Config 2 & Config 3 & Config 4 & Config 5 \\
\hline \multirow{7}{*}{ Hyperparameters } & $\begin{array}{l}\text { Hidden dimension in } \\
\text { LSTM }\end{array}$ & $\begin{array}{l}\text { See Table } \\
\text { S1 }\end{array}$ & 220 & 389 & 256 & 180 \\
\hline & $\begin{array}{l}\text { Hidden dimension in } \\
\text { attention }\end{array}$ & $\begin{array}{l}\text { See Table } \\
\text { S1 }\end{array}$ & 189 & 194 & 256 & 369 \\
\hline & $\begin{array}{l}\text { Number of heads in } \\
\text { attention }\end{array}$ & $\begin{array}{l}\text { See Table } \\
\text { S1 }\end{array}$ & 27 & 47 & 30 & 41 \\
\hline & $\begin{array}{l}\text { Regularizer of L2 loss } \\
\text { in attention }\end{array}$ & $\begin{array}{l}\text { See Table } \\
\text { S1 }\end{array}$ & $10 \mathrm{E}-5$ & $3 \mathrm{E}-4$ & 0.001 & $10 \mathrm{E}-5$ \\
\hline & $\begin{array}{l}\text { Regularizer of } \\
\text { penalization in attention }\end{array}$ & $\begin{array}{l}\text { See Table } \\
\text { S1 }\end{array}$ & $10 \mathrm{E}-5$ & $1.3 \mathrm{E}-4$ & 0.001 & $7.2 \mathrm{E}-4$ \\
\hline & $\begin{array}{l}\text { Dropout rate of input } \\
\text { layer }\end{array}$ & $\begin{array}{l}\text { See Table } \\
\text { S1 }\end{array}$ & 0.1 & 0.116 & 0.2 & 0.1 \\
\hline & $\begin{array}{l}\text { Dropout rate of hidden } \\
\text { layers }\end{array}$ & $\begin{array}{l}\text { See Table } \\
\text { S1 }\end{array}$ & 0.1 & 0.133 & 0.5 & 0.1 \\
\hline \multirow{12}{*}{ Performance } & Accuracy & 0.759 & 0.753 & 0.765 & 0.743 & 0.761 \\
\hline & Gorodkin & 0.707 & 0.701 & 0.715 & 0.688 & 0.711 \\
\hline & MCC-Nucleus & 0.733 & 0.722 & 0.739 & 0.716 & 0.725 \\
\hline & MCC-Cytoplasm & 0.550 & 0.538 & 0.563 & 0.536 & 0.549 \\
\hline & MCC-Extracellular & 0.894 & 0.896 & 0.897 & 0.887 & 0.896 \\
\hline & MCC-Mitochondrion & 0.811 & 0.832 & 0.832 & 0.783 & 0.823 \\
\hline & MCC-Cell membrane & 0.695 & 0.677 & 0.683 & 0.679 & 0.696 \\
\hline & MCC-ER & 0.568 & 0.571 & 0.589 & 0.543 & 0.602 \\
\hline & MCC-Plastid & 0.908 & 0.919 & 0.896 & 0.868 & 0.901 \\
\hline & MCC-Glogi apparatus & 0.449 & 0.455 & 0.535 & 0.449 & 0.464 \\
\hline & MCC-Lysosome & 0.149 & 0.096 & 0.157 & 0.062 & 0.208 \\
\hline & MCC-Peroxisome & 0.449 & 0.412 & 0.423 & 0.370 & 0.412 \\
\hline
\end{tabular}

Config 1: Each sub-model uses its own optimized hyperparameters.

Config 2: All sub-models use the same but not the final optimum hyperparameters, in this case, the hyperparameters in submodel 2.

Config 3: All sub-models use the same but not the final optimum hyperparameters, in this case, the hyperparameters in submodel 3.

Config 4: All sub-models use the same random hyperparameters.

Config 5: All sub-models use the same final optimum hyperparameters.

Performance is measured by accuracy, Gorodkin value, and Matthew's correlation coefficient (MCC).

using the randomly selected hyperparameters. We also conducted an experiment to test the performance of individual models using the final optimum hyperparameters. The results are listed in Table 3.5. Comparing the results in Table 3.4, the ensemble models generally have a better performance than individual models.

The UniLoc-train dataset was divided into 8 folds. Eight models were trained where each of them used 7 folds as training and 1 fold was left for evaluation. The final MULocDeep model is the ensemble of eight models, and the prediction of a protein is the average of predictions from these eight models. All these models used the same final optimized hyperparameters. To train each of these models, the lv1_train and lv2_train in 
Table 3.5. Performance of Single Models on Different Hyperparameter Configurations

\begin{tabular}{|l|l|l|l|l|l|l|l|l|l|}
\hline \multicolumn{2}{|c|}{} & M1 & M2 & M3 & M4 & M5 & M6 & M7 & M8 \\
\hline \multirow{5}{*}{ Performance } & Accuracy & 0.735 & 0.724 & 0.716 & 0.726 & 0.729 & 0.729 & 0.730 & 0.717 \\
\cline { 2 - 10 } & Gorodkin & 0.679 & 0.666 & 0.656 & 0.669 & 0.672 & 0.673 & 0.673 & 0.657 \\
\cline { 2 - 10 } & MCC-Nucleus & 0.700 & 0.674 & 0.683 & 0.709 & 0.692 & 0.715 & 0.685 & 0.682 \\
\cline { 2 - 10 } & MCC-Cytoplasm & 0.528 & 0.466 & 0.468 & 0.505 & 0.507 & 0.505 & 0.490 & 0.482 \\
\cline { 2 - 10 } & MCC-Extracellular & 0.879 & 0.867 & 0.879 & 0.869 & 0.879 & 0.874 & 0.877 & 0.870 \\
\cline { 2 - 10 } & MCC-Mitochondrion & 0.770 & 0.802 & 0.801 & 0.749 & 0.783 & 0.767 & 0.779 & 0.777 \\
\cline { 2 - 10 } & MCC-Cell membrane & 0.678 & 0.688 & 0.621 & 0.679 & 0.678 & 0.654 & 0.686 & 0.640 \\
\cline { 2 - 10 } & MCC-ER & 0.544 & 0.547 & 0.540 & 0.505 & 0.505 & 0.524 & 0.562 & 0.522 \\
\cline { 2 - 10 } & MCC-Plastid & 0.874 & 0.874 & 0.808 & 0.871 & 0.858 & 0.883 & 0.868 & 0.845 \\
\cline { 2 - 10 } & MCC-Glogi apparatus & 0.323 & 0.316 & 0.389 & 0.366 & 0.361 & 0.306 & 0.394 & 0.329 \\
\cline { 2 - 10 } & MCC-Lysosome & 0.095 & 0.137 & 0.144 & 0.173 & 0.112 & 0.166 & 0.111 & 0.077 \\
\cline { 2 - 9 } & MCC-Peroxisome & 0.400 & 0.324 & 0.300 & 0.189 & 0.342 & 0.452 & 0.295 & 0.481 \\
\hline
\end{tabular}

Each sub-model (M1-M8) used the same final optimum hyperparameters.

the UniLoc-train dataset were utilized iteratively. Specifically, we trained them using the samples with only subcellular localization labels (lv1_train) for 1 epoch, and then trained another epoch using the samples with suborganelle localization labels (lv2_train). This process was repeated 80 times for each of the eight models. When a training sample had both suborganelle and subcellular (inferred from suborganelle) annotation, each element in the matrix (Figure 3.1) yielded a loss using a binary cross entropy loss function after a sigmoid activation function (Lost 1). The maximum prediction score under each organelle (each column in the matrix in Figure 3.2) was extracted and went through another binary cross entropy (Lost 2). If a training sample only had the subcellular localization information, the Lost 1 was not used, only the Lost 2 was calculated after the Max-Pooling operation.

The training process was written using the Keras package (version 2.3.0) and run using a NVIDIA GeForce RTX 2080 Ti GPU. The training time for the MULocDeep model was roughly $2 \mathrm{~min}$ for one epoch.

The thresholds for predicting suborganelle localizations were determined from a cross-validation results by UniLoc-train at the suborganelle level with different prediction thresholds (if the prediction output is above the threshold, a positive label is 
predicted), which is shown in Table 3.2. The default threshold is 0.5 , and we tuned it in a way that favors positive predictions (high recall) based on the results in Table 3.2. In particular, we only tuned the thresholds in the range below 0.5 to achieve the highest MCC. The same thresholds determination process of MULocDeep were also applied to the training of the non-redundant model.

\subsubsection{Variant model}

The purpose of variant model is to compare with other methods which only predict localization at either subcellular level or suborganellar level. The architecture of a variant
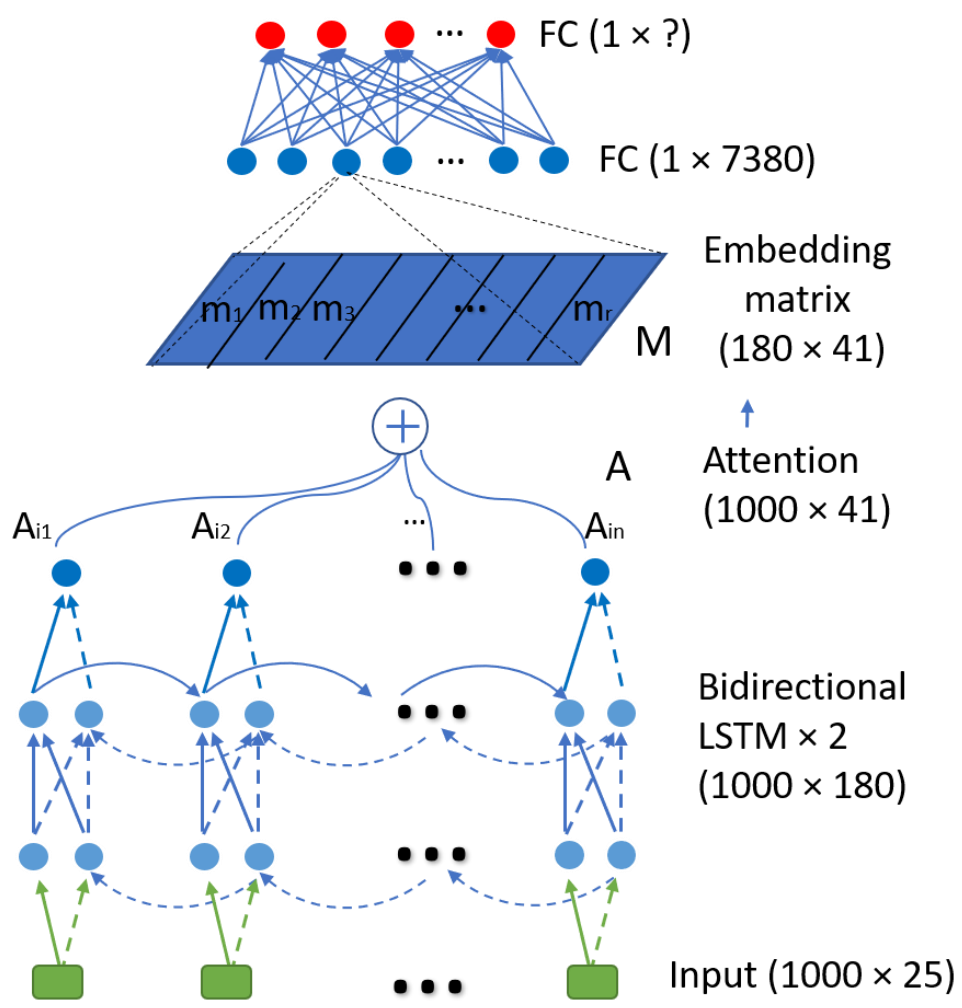

Figure 3.4. Deep learning architecture of the variant model. The cell hierarchical matrix is replaced by a fully connected layer with the size equal to the number of classes to predict. 
MULocDeep model is shown in Figure 3.4. It is the same as the MULocDeep model before the fully connected layer ( 1 by 80 ) in Figure 1 . The difference is that instead of reshaping the vector into a matrix in Figure 3.1, we used the vector as output directly for the variant model. The length of the vector is adjusted according to the method we compared with, either to predict subcellular localizations (equivalent to one row in the matrix in Figure 3.1) or to predict suborganellar localizations under one specific organelle (equivalent to one column in the matrix in Figure 3.1). Thus, when training a variant model, we only used the corresponding training data provided by the specific methods that we wanted to compare with. The variant model also used the same final optimized hyperparameters as in the MULocDeep model. We also needed to pick the combination of either a softmax activation function with a categorical cross entropy loss function, or a sigmoid activation function with a binary cross entropy loss function, based on the situation if the other method was a single-label or multi-label prediction method, respectively. 


\section{CHAPTER 4. PERFORMANCE EVALUATION AND MECHANISM INTERPRETATION}

In this chapter, we first introduce the datasets used for localization prediction performance evaluating and comparison. Then, we show the evaluating results by different methods under different scenarios. Finally, we illustrate the localization mechanism interpretation ability of MULocDeep powered by the attention mechanism.

\subsection{Testing datasets}

Besides the UniLoc-test dataset which is already introduced in the UniLoc dataset section in Chapter 3, there are other testing datasets focusing on different scenarios.

\subsubsection{The three-species mitochondrial proteome dataset (Mito3).}

This dataset contains the mitochondrial proteome extracted from three plant species and was generated by our collaborators in Leibniz University as follows. First, mitochondria were isolated from an Arabidopsis thaliana cell culture, Solanum tuberosum tubers, and Vicia faba roots. The numbers of biological replicates are 3, 7, and 3 for Arabidopsis thaliana, Solanum tuberosum (potato) and Vicia faba (bean), respectively. Then, the mitochondrial proteins of the three species were identified and quantified using shotgun mass spectrometry according to Thal et al. [113]. The generated raw files were analysed with the Proteome Discoverer software (Thermo Fisher Scientific, Dreieich, Germany) using the Mascot (Matrix Science, London, UK) search 
engine against in-house protein sequence databases, depending on the analyzed species: Arabidopsis thaliana, Solanum tuberosum and Vicia faba spectra were queried against the TAIR 10 database, the Solanum tuberosum protein database and the Medicago truncatula protein database 4.0, respectively. A total of 8002 proteins from these three species were identified in this step. Finally, the proteins of Vicia and Solanum were assigned to their Arabidopsis orthologues by pairwise BLAST searches of the underlying sequence datasets. The identified proteins from the three species with the respective Arabidopsis orthologues are shown in Supplementary Data 1-3 of the MULocDeep paper. In total, 4778 unique mitochondrial proteins were identified. Since this dataset is used for localization classifier evaluation at the subcellular level (for evaluation of predicting if a protein is mitochondrial or not), we balanced it by collecting 8002 plant proteins that were labelled as non-mitochondrial in the UniProt database (release 2020_04). The proteins in this dataset were not used in training the model. To perform a more rigorous test, we applied blastp [45] to align the Mito3 dataset against the UniLoc-train-40nr dataset. If a hit has an alignment coverage higher than $80 \%$ of the shorter sequence and a $40 \%$ sequence identity or $10^{-5} \mathrm{E}$-value, it will be removed. The remaining sequences in Mito3 were kept and formed Mito3-40nr (1929 positive samples and 1450 negative samples).

\subsubsection{External datasets.}

We used the datasets provided by other methods to train and test a variant model for a rigorous evaluation of methodology. Two datasets (SM424-18 and SubMitoPred) are from the DeepMito method which focuses on the prediction of sub-mitochondrial protein 
localization. One dataset (DeepLoc dataset) is from the DeepLoc method which predicts 10 main localizations at subcellular level. The SM424-18 dataset was derived from the UniProt database (release 2018_02). They filtered the proteins by selecting all nonfragment protein sequences with evidence at the protein level for experimentally determined subcellular localization in one of the four sub-mitochondrial compartments: outer membrane, intermembrane space, inner membrane and matrix. They further reduced the redundancy using the CD-HIT program so that 424 mitochondrial proteins remained sharing at most $40 \%$ sequence identity. The SubMitoPred dataset was derived from the UniProt database release 2014_10. The protein selection criteria were: fulllength proteins $>50$ residues, single experimental sub-mitochondrial localization, and internal redundancy reduced at $40 \%$ sequence identity using CD-HIT. The dataset comprises 570 mitochondrial proteins distributed in the same four compartments as in the SM424-18 dataset. Both datasets were split into folds, 10 for the SM424-18 dataset and 5 for the SubMitoPred dataset. The category details can be found in Table 4.1. The DeepLoc dataset was extracted from the UniProt database, release 2016_04. It contains proteins from experimentally annotated 10 main compartments in eukaryotic cells. A total of 13858 proteins were obtained and the training and testing samples are marked. The number of proteins in each compartment of the DeepLoc dataset is shown in Table 4.2. 
Table 4.1. Statistics from the Datasets Used in the DeepMito Method

\begin{tabular}{|c|c|c|}
\hline \multirow{2}{*}{ Compartments } & \multicolumn{2}{|c|}{ Datasets } \\
\cline { 2 - 3 } & SM424-18 & SubMitoPred \\
\hline Outer membrane & 74 & 282 \\
\hline Inner membrane & 190 & 32 \\
\hline Intermembrane space & 25 & 174 \\
\hline Matrix & 135 & 570 \\
\hline Total & 424 & \\
\hline
\end{tabular}

Table 4.2. Number of proteins in each localization class in the DeepLoc dataset

\begin{tabular}{|c|c|}
\hline Localization & No. of proteins \\
\hline Nucleus & 4043 \\
\hline Cytoplasm & 2542 \\
\hline Extracellular & 1973 \\
\hline Mitochondrion & 1510 \\
\hline Cell membrane & 1340 \\
\hline Endoplasmic reticulum & 862 \\
\hline Plastid & 757 \\
\hline Golgi apparatus & 356 \\
\hline Lysosome/Vacuole & 321 \\
\hline Peroxisome & 154 \\
\hline
\end{tabular}

\subsection{Evaluation criteria}

We used accuracy (ACC), Matthew's correlation coefficient (MCC) [114], recall, precision, area under the receiver operating characteristic curve (ROC_auc), and area under precision \& recall curve (P\&R_auc) to evaluate our method and compare it with others. For unbalanced datasets, measurements such as ACC, recall and precision would 
introduce bias and overestimate a method's performance. MCC considers true and false positives and negatives, and is generally regarded as a balanced measure even if the classes are of very different sizes [115]. The definitions of ACC, MCC, recall and precision are listed in Eqs. 22-25:

$$
\begin{gathered}
A C C=\frac{T P+T N}{T P+T N+F P+F N} \\
M C C=\frac{T P * T N-F P * F N}{\sqrt{(T P+F P)(T P+F N)(T N+F P)(T N+F N)}} \\
\text { recall }=\frac{T P}{T P+F N} \\
\text { precision }=\frac{T P}{T P+F P}
\end{gathered}
$$

where $T P, F P, T N, F N$ are true positive, false positive, true negative and false negative prediction, respectively. Measurements such as MCC, recall, precision and ACC can be used in binary prediction cases.

\subsection{Comparison of performance in localization prediction}

Firstly, we compared the protein localization prediction of MULocDeep with other available methods on four benchmark datasets provided by us, the Mito3, the UniLocsub, the UniLoc-single and the UniLoc-multi datasets. We summarized a list of localization classifiers regarding their scope (target localizations), availabilities (web server or local tool) and the performance on these datasets. We have investigated many other methods, but they were excluded from the comparison because they were either unavailable, not working properly at the time of test, or only accept a single sequence for 
Table 4.3. Evaluation and Comparison of Protein Localization Prediction Methods.

\begin{tabular}{|c|c|c|c|c|c|c|c|c|c|c|}
\hline \multicolumn{11}{|c|}{ Mitochondrion localization prediction using the Mito3 dataset: } \\
\hline \multirow[t]{2}{*}{ Method } & \multirow[t]{2}{*}{ Scope } & \multirow[t]{2}{*}{ AVAIL } & \multirow[t]{2}{*}{ Subcellular } & \multirow[t]{2}{*}{ Suborganellar } & \multicolumn{6}{|c|}{ Assessments } \\
\hline & & & & & ROC_auc & P\&R_auc & MCC & Recall & Prec & Acc \\
\hline MULocDeep & $1-10$ & W\&L & \multirow{8}{*}{ Mitochondrion } & I & $0.74 / 0.69$ & $0.79 / 0.77$ & $0.39 / 0.31$ & $0.30 / 0.29$ & $0.94 / 0.90$ & $0.64 / 0.57$ \\
\hline MULoc & 4 & W\&L & & 1 & 0.78 & 0.82 & 0.42 & 0.52 & 0.85 & 0.67 \\
\hline DeepLoc & $1-10$ & W\&L & & I & 0.70 & 0.63 & 0.39 & 0.35 & 0.76 & 0.75 \\
\hline TargetP v5 & $3,4,7$ & W\&L & & I & 0.72 & 0.79 & 0.23 & 0.13 & 0.97 & 0.50 \\
\hline MitoFates & 4 & W\&L & & I & 0.63 & 0.71 & 0.22 & 0.15 & 0.90 & 0.50 \\
\hline SherLoc2 & $1-11$ & W & & I & 0.68 & 0.73 & 0.22 & 0.18 & 0.85 & 0.51 \\
\hline MultiLoc2 & $1-11$ & W\&L & & I & 0.68 & 0.73 & 0.22 & 0.15 & 0.90 & 0.50 \\
\hline Predotar & $4,6,7$ & $\mathrm{~W}$ & & I & 0.60 & 0.68 & 0.24 & 0.21 & 0.87 & 0.53 \\
\hline \multicolumn{11}{|c|}{ Suborganellar localization prediction using UniLoc-sub dataset: } \\
\hline \multirow{10}{*}{ MULocDeep } & \multirow{10}{*}{$1-10$} & \multirow{10}{*}{ W\&L } & \multirow{4}{*}{ Mitochondrion } & Inner membrane & $0.83 / 0.75$ & $0.74 / 0.70$ & $0.41 / 0.37$ & $0.92 / 0.78$ & $0.55 / 0.58$ & $0.65 / 0.67$ \\
\hline & & & & Outer membrane & $0.71 / 0.49$ & $0.67 / 0.27$ & $0.67 / 0.18$ & $0.59 / 0.13$ & $0.86 / 0.50$ & $0.91 / 0.82$ \\
\hline & & & & Matrix & $\mathbf{0 . 8 8} / 0.76$ & $0.81 / 0.53$ & $\mathbf{0 . 7 1} / 0.42$ & $0.86 / 0.64$ & $0.72 / 0.51$ & $\mathbf{0 . 8 9 / 0 . 7 7}$ \\
\hline & & & & Intermem. space & $0.83 / 0.58$ & $0.56 / 0.16$ & $0.38 / 0.15$ & $0.31 / 0.50$ & $0.56 / 0.16$ & $0.91 / 0.71$ \\
\hline & & & \multirow[t]{2}{*}{ Golgi apparatus } & Trans-Golgi & $0.72 / 0.59$ & $0.92 / 0.85$ & $0.38 / 0.26$ & $0.63 / 0.23$ & $0.92 / 1.00$ & $0.67 / 0.41$ \\
\hline & & & & Cis-Golgi & $\mathbf{0 . 8 1} / 0.49$ & $\mathbf{0 . 5 8} / 0.26$ & $\mathbf{0 . 4 8 / 0 . 0 6}$ & $\mathbf{0 . 6 4} / 0.09$ & $0.58 / 0.33$ & $\mathbf{0 . 8 0 / 0 . 7 4}$ \\
\hline & & & \multirow[t]{4}{*}{ Plastid (chloroplast) } & Membrane & $\mathbf{0 . 5 4} / 0.36$ & $\mathbf{0 . 5 1} / 0.25$ & $0.45 / 0.12$ & $0.38 / 0.21$ & $0.82 / 0.21$ & $0.79 / 0.54$ \\
\hline & & & & Stroma & $0.80 / 0.60$ & $0.76 / 0.43$ & $0.63 / 0.13$ & $0.67 / 0.52$ & $0.82 / 0.40$ & $\mathbf{0 . 8 4} / 0.59$ \\
\hline & & & & Thylakoid lumen & $0.86 / 0.82$ & $0.49 / 0.29$ & $0.52 / 0.30$ & $0.50 / 0.67$ & $0.60 / 0.22$ & $\mathbf{0 . 9 4 / 0 . 8 0}$ \\
\hline & & & & Thylakoid mem. & $0.87 / 0.72$ & $0.84 / 0.68$ & $0.38 / 0.30$ & $\mathbf{0 . 8 6} / 0.59$ & $0.50 / 0.53$ & $0.65 / 0.67$ \\
\hline \multirow[t]{4}{*}{ DeepMito } & 4 & W\&L & Mitochondrion & Inner membrane & 0.78 & 0.66 & 0.48 & 0.82 & 0.63 & 0.73 \\
\hline & & & & Outer membrane & 0.80 & 0.57 & 0.62 & 0.65 & 0.72 & 0.89 \\
\hline & & & & Matrix & 0.78 & 0.59 & 0.60 & 0.61 & 0.76 & 0.86 \\
\hline & & & & Intermem. space & 0.85 & 0.53 & 0.65 & 0.75 & 0.63 & 0.93 \\
\hline SubGolgi v2 & 8 & $\mathrm{~W}$ & Golgi apparatus & Trans-Golgi & 0.77 & 0.92 & 0.47 & 0.85 & 0.88 & 0.80 \\
\hline & & & & Cis-Golgi & 0.77 & 0.45 & 0.47 & 0.63 & 0.58 & 0.80 \\
\hline TetraMito & 4 & $\mathrm{~W}$ & Mitochondrion & Inner membrane & 0.61 & 0.55 & 0.17 & 0.52 & 0.51 & 0.59 \\
\hline & & & & Outer membrane & 0.71 & 0.59 & 0.45 & 0.46 & 0.62 & 0.85 \\
\hline & & & & Matrix & 0.62 & 0.29 & 0.06 & 0.50 & 0.26 & 0.55 \\
\hline & & & Plastid (chloroplast) & Membrane & 0.43 & 0.26 & -0.09 & 0.29 & 0.23 & 0.51 \\
\hline Schloro & 7 & W & & Stroma & 0.50 & 0.32 & 0.00 & 0.00 & 0.00 & 0.67 \\
\hline & & & & Thylakoid lumen & 0.50 & 0.07 & 0.00 & 0.00 & 0.00 & 0.92 \\
\hline & & & & Thylakoid mem. & 0.53 & 0.37 & 0.03 & 0.65 & 0.36 & 0.47 \\
\hline Subcellular lo & on predi & 1 using $U_{t}$ & oc-single dataset: & & & & & & & \\
\hline & & & Nucleus & I & $0.97 / 0.94$ & $0.93 / 0.85$ & $0.81 / 0.68$ & $0.91 / 0.87$ & $0.82 / 0.69$ & $\mathbf{0 . 9 3 / 0 . 8 6}$ \\
\hline & & & Cytoplasm & I & $\mathbf{0 . 9 4} / 0.86$ & $\mathbf{0 . 8 3} / 0.66$ & $0.71 / 0.53$ & $\mathbf{0 . 8 3} / 0.74$ & $0.74 / 0.59$ & $\mathbf{0 . 8 9 / 0 . 8 1}$ \\
\hline & & & Extracellular & I & $0.99 / 0.98$ & $0.92 / 0.83$ & $0.87 / 0.82$ & $0.92 / 0.89$ & $0.86 / 0.80$ & $0.97 / 0.96$ \\
\hline MULocDeep & $1-10$ & W\&L & Mitochondrion & I & $0.98 / 0.94$ & $\mathbf{0 . 9 2} / 0.82$ & $0.87 / 0.74$ & $0.84 / 0.70$ & $0.92 / 0.82$ & $\mathbf{0 . 9 8 / 0 . 9 6}$ \\
\hline & & & Cell membrane & I & $\mathbf{0 . 9 6 / 0 . 9 1}$ & $0.89 / 0.76$ & $0.77 / 0.64$ & $0.86 / 0.76$ & $0.76 / 0.65$ & $\mathbf{0 . 9 4 / 0 . 9 0}$ \\
\hline & & & $\overline{E R}$ & I & $\mathbf{0 . 9 6 / 0 . 8 7}$ & $\mathbf{0 . 8 2} / 0.50$ & $\mathbf{0 . 8 0} / 0.48$ & $0.75 / 0.41$ & $0.88 / 0.62$ & $\mathbf{0 . 9 8 / 0 . 9 5}$ \\
\hline & & & Plastid & l & $\mathbf{0 . 9 9 / 0 . 9 4}$ & $\mathbf{0 . 8 6} / 0.68$ & $\mathbf{0 . 8 3} / 0.71$ & $\mathbf{0 . 8 2 / 0 . 6 4}$ & $0.85 / 0.79$ & $0.99 / 0.99$ \\
\hline & & & Golgi apparatus & l & $\mathbf{0 . 9 5} / 0.89$ & $0.71 / 0.49$ & $\mathbf{0 . 7 0 / 0 . 4 4}$ & $0.67 / 0.57$ & $0.75 / 0.37$ & $\mathbf{0 . 9 8 / 0 . 9 6}$ \\
\hline & & & Lysosome & I & $0.97 / 0.80$ & $0.63 / 0.10$ & $0.66 / 0.14$ & 0.50/0.10 & $0.87 / 0.20$ & $0.99 / 0.99$ \\
\hline & & & Peroxisome & I & $0.98 / 0.94$ & $\mathbf{0 . 7 5} / 0.53$ & $0.75 / 0.54$ & $0.57 / 0.38$ & $1.00 / 0.78$ & $0.99 / 0.99$ \\
\hline & & & Nucleus & I & 0.96 & 0.91 & 0.78 & 0.85 & 0.83 & 0.91 \\
\hline & & & Cytoplasm & I & 0.90 & 0.78 & 0.64 & 0.75 & 0.72 & 0.86 \\
\hline & & & Extracellular & I & 0.97 & 0.85 & 0.81 & 0.84 & 0.83 & 0.95 \\
\hline DeepLoc & $1-10$ & W\&L & Mitochondrion & I & 0.97 & 0.89 & 0.82 & 0.86 & 0.82 & 0.97 \\
\hline & & & Cell membrane & I & 0.92 & 0.80 & 0.72 & 0.67 & 0.87 & 0.93 \\
\hline & & & ER & I & 0.93 & 0.75 & 0.70 & 0.75 & 0.69 & 0.96 \\
\hline & & & Plastid & I & 0.96 & 0.76 & 0.74 & 0.81 & 0.68 & 0.98 \\
\hline & & & Golgi apparatus & I & 0.93 & 0.66 & 0.66 & 0.62 & 0.72 & 0.98 \\
\hline & & & Lysosome & I & 0.88 & 0.32 & 0.35 & 0.47 & 0.28 & 0.98 \\
\hline & & & Peroxisome & I & 0.93 & 0.55 & 0.52 & 0.54 & 0.52 & 0.99 \\
\hline
\end{tabular}

The upper part of the table uses our Mito3 dataset to evaluate the performance of the mitochondrial protein prediction; the middle part uses the UniLoc-sub dataset to evaluate the performance at the suborganelle level prediction; and the lower part uses the UniLoc-single dataset to evaluate the performance at the subcellular level. Availability (AVAIL) is either through a web server (W) or a local tool (L). The prediction scope includes compartments in: 1 . nucleus; 2. cytoplasm; 3. extracellular; 4. mitochondrion; 5. cell membrane; 6. endoplasmic reticulum; 7. plastid/chloroplast; 8. Golgi apparatus; 9. lysosome/vacuole; 10. peroxisome; 11. plasma membrane. Criteria of assessment are ROC_auc (area under the receiver operating characteristic curve), P\&R auc (area under precision \& recall curve), MCC (Matthew's correlation coefficient), recall, precision, and accuracy. Localization categories with less than 6 samples were removed. The performance after the slash is from the MULocDeep method trained using non-redundant dataset (UniLoc-train-4Onr). It was evaluated using the same test datasets, except the upper part of the table, where nonredundant MULocDeep model was evaluated using Mito3-40nr.

web submission. The Mito3 dataset was used to evaluate the performance of different

classifiers for mitochondrial proteins (Table 4.3, upper part). The UniLoc-sub dataset was 
used to test the suborganellar localization prediction for Golgi, mitochondrion, and chloroplast (Table 4.3, middle part). The UniLoc-single dataset was used to compare with DeepLoc for the 10 main subcellular localization predictions (Table 4.3 lower part). Since the UniLoc-test dataset mostly consisted of proteins created after 2018 in the UniProt database, we assume that for most of the comparing methods, the same protein sequence did not appear in their training sets. Some new methods, e.g., DeepMito, may not guarantee that, but this would be to the advantage of these methods instead of ours since no sequence in the UniLoc-test dataset was used in training our model. Hence, the comparison in Table 4.3 can be regarded as an evaluation from the tool perspective, which mimics the actual usage that cares about the prediction performance instead of the homology issue. To evaluate the generalization of our method, the performance of MULocDeep model trained using the non-redundant training set (UniLoc-train-40nr) is also shown in Table 4.3 separated by a slash.

Among the six measurements of the performance, the ROC_auc (area under the receiver operating characteristic curve) and $\mathrm{P} \& \mathrm{R} \_$auc (area under precision $\&$ recall curve) are the most important criteria from the method perspective as they reflect accuracies in a continuous range of thresholds for a binary prediction while the other measurements are affected by the chosen thresholds. In the upper part of Table 4.3, MULoc, a mitochondrion-specific method developed by our lab has the highest score in most of the measurements. Except for this, MULocDeep has more than half of the measurements better than any other method in a pair-wise comparison. Especially, the ROC_auc and P\&R_auc are consistently higher in MULocDeep than others. At the suborganellar level prediction (Table 4.3 middle), MULocDeep is consistently better in 
predicting subchloroplastic proteins. It is also generally better in predicting cis-Golgi network proteins, mitochondrion matrix and outer membrane proteins. DeepMito [105] is better for the prediction of the mitochondrion intermembrane space proteins and SubGolgi v2 [96] is better in trans-Golgi network protein prediction. It is noted that all the better methods than MULocDeep are organelle specific, which suggests tuning models for a single organelle may have some advantages. In the lower part of Table 4.3, MULocDeep achieved a higher score in most of the measurements than DeepLoc. It is worth mentioning in Table 4.3 that MULocDeep's performance using the non-redundant training set did not drop significantly compared to the model using the redundant training set in terms of ROC_auc and P\&R_auc which indicates a good generalization of MULocDeep.

The UniLoc-multi dataset was used to compare with pLoc-mEuk [116], which is a subcellular localization prediction method for multi-label eukaryotic proteins (i.e., predicting multiple labels simultaneously). All the proteins in the UniLoc-multi dataset have more than one localization annotation. The comparison result for the multi-label localization prediction is shown in Table 4.4. We used the exact match as the criterion to evaluate the overall performance of each method, and MCC, recall, precision, and accuracy to evaluate individual classes. According to Table 4.4, MULocDeep, either the redundant or non-redundant model, has significantly better individual scores than pLocmEuk in almost every case. 
Table 4.4. Comparison of Multi-label Protein Localization Prediction Performance.

\begin{tabular}{|c|c|c|c|c|c|c|}
\hline \multicolumn{7}{|c|}{ UniLoc-multi dataset: } \\
\hline Method & Localization & $\mathrm{MCC}$ & Recall & Prec & $\mathrm{ACC}$ & Exact match \\
\hline \multirow{8}{*}{ MULocDeep } & Nucleus & $0.59 / 0.53$ & $0.66 / 0.62$ & $0.99 / 0.97$ & $0.76 / 0.72$ & \multirow{8}{*}{$\begin{array}{c}\mathbf{2 4 3} / 144 \\
(35 \% / 21 \%)\end{array}$} \\
\hline & Cytoplasm & $\mathbf{0 . 3 6} / 0.21$ & $0.68 / 0.59$ & $\mathbf{0 . 9 5} / 0.92$ & $0.70 / 0.61$ & \\
\hline & Extracellular & $0.60 / 0.29$ & $0.60 / 0.40$ & $0.60 / 0.22$ & $0.99 / 0.99$ & \\
\hline & Mitochondrion & $\mathbf{0 . 4 7 / 0 . 2 3}$ & $\mathbf{0 . 4 0 / 0 . 2 0}$ & $\mathbf{0 . 6 0} / 0.33$ & $0.96 / 0.95$ & \\
\hline & Cell membrane & $0.74 / 0.51$ & $0.73 / 0.54$ & $0.85 / 0.64$ & $0.93 / 0.86$ & \\
\hline & ER & $\mathbf{0 . 7 0} / 0.41$ & $\mathbf{0 . 5 3} / 0.22$ & $1.00 / 0.87$ & $0.94 / 0.90$ & \\
\hline & Golgi apparatus & $0.73 / 0.33$ & $0.58 / 0.32$ & $\mathbf{0 . 9 8} / 0.47$ & $0.96 / 0.90$ & \\
\hline & Lysosome & $0.64 / 0.23$ & $0.47 / 0.12$ & $0.89 / 0.50$ & $0.99 / 0.98$ & \\
\hline \multirow{8}{*}{ pLoc-mEuk } & Nucleus & 0.38 & 0.57 & 0.89 & 0.65 & \multirow{8}{*}{$\begin{array}{c}161 \\
(23 \%)\end{array}$} \\
\hline & Cytoplasm & 0.28 & 0.52 & 0.95 & 0.57 & \\
\hline & Extracellular & 0.09 & 0.20 & 0.05 & 0.97 & \\
\hline & Mitochondrion & 0.28 & 0.26 & 0.36 & 0.94 & \\
\hline & Cell membrane & 0.36 & 0.34 & 0.58 & 0.83 & \\
\hline & ER & 0.43 & 0.32 & 0.70 & 0.89 & \\
\hline & Golgi apparatus & 0.33 & 0.26 & 0.54 & 0.90 & \\
\hline & Lysosome & 0.11 & 0.05 & 0.25 & 0.97 & \\
\hline
\end{tabular}

Criteria are Matthew's correlation coefficient (MCC), recall, precision (Prec), accuracy (ACC), and exact match. The exact match means that there is no difference in all the organelle labels between the predicted ones and the experimental ones. The best score in each sub-category is shown in bold. Localization categories with less than 6 samples were removed. The performance after the slash is from the MULocDeep method trained using nonredundant dataset (UniLoc-train-40nr).

To evaluate different approaches from the method perspective under a rigorous condition, we created a variant of the MULocDeep model. We used the variant model to compare with different methods individually at both subcellular and suborganellar levels. At the suborganellar level, only a few methods have provided clearly separated datasets for training and testing, which makes it difficult to make a fair comparison. Here we compared with DeepMito [105], a recently published deep learning method for submitochondrial protein localization prediction. A variant model was trained using the same DeepMito datasets provided by the DeepMito paper. The output layer was a 4dimensional vector representing four target compartments (outer membrane, inner membrane, intermembrane space and matrix) in mitochondria as in DeepMito. Processing 
the data as in the DeepMito method, the SM424-18 dataset and the SubMitoPred dataset were split into 10 and 5 folds, respectively. The comparison was based on the MCC of different compartments from the cross validation as reported in the DeepMito paper [105]. MULocDeep performed better than DeepMito for every mitochondrial compartment in both datasets (Table 4.5).

For protein localization prediction at the subcellular level, the DeepLoc dataset was originally used to compare between DeepLoc and eight other methods [103]. The results show that the DeepLoc method outperformed other methods in terms of both accuracy and Gorodkin value [117]. To demonstrate the effectiveness of our method in predicting

Table 4.5. Comparison of Method Effectiveness between MULocDeep and DeepMito.

\begin{tabular}{|l|l|l|l|l|l|l|}
\hline Dataset & Method & $\begin{array}{l}\text { Feature/CV } \\
\text { method }\end{array}$ & MCC(O) & MCC(I) & MCC(T) & MCC(M) \\
\hline \multirow{5}{*}{ SM424-18 } & \multirow{5}{*}{ DeepMito } & SEQ & 0.17 & 0.15 & 0.13 & 0.07 \\
\cline { 3 - 7 } & & PROP & 0.17 & 0.07 & 0.22 & 0.13 \\
\cline { 3 - 7 } & & PSSM & 0.51 & 0.47 & 0.42 & 0.57 \\
\cline { 3 - 7 } & & SEQ+PROP & 0.16 & 0.07 & 0.55 & 0.09 \\
\cline { 3 - 7 } & & PSSM+PROP & 0.46 & 0.47 & 0.53 & 0.65 \\
\cline { 2 - 7 } & MULocDeep & PSSM+PROP & $\mathbf{0 . 5 3}$ & $\mathbf{0 . 5 9}$ & $\mathbf{0 . 5 9}$ & $\mathbf{0 . 6 7}$ \\
\hline & SubMitoPred & RS & 0.42 & 0.34 & 0.19 & 0.51 \\
\cline { 2 - 7 } & DeepMito & RS & 0.45 & 0.68 & 0.54 & 0.79 \\
\cline { 2 - 7 } & DeepMito & CL & 0.42 & 0.60 & 0.46 & 0.76 \\
\cline { 2 - 7 } & MULocDeep & RS & $\mathbf{0 . 6 7}$ & $\mathbf{0 . 7 6}$ & $\mathbf{0 . 6 7}$ & 0.79 \\
\hline
\end{tabular}

The comparison is at the sub-organellar level. Four target compartments are "O": Outer membrane, "I": Inner membrane, "T": Intermembrane space, and "M": Matrix. Features used include one-hot encoding residue (SEQ), physico-chemical properties (PROP), and position-specific scoring matrix (PSSM). Cross-validation (CV) methods include randomly splitting the dataset (RS) and confining local similarity into the same cross-validation set (CL). The assessment is based on Mathew's correlation coefficient (MCC).

protein localization at the subcellular level, like what we did for the sub-mitochondrial prediction, the variant model with ten target localizations (nucleus, cytoplasm, 
extracellular, mitochondrion, cell membrane, endoplasmic reticulum, plastid, Golgi apparatus, lysosome, and peroxisome) was trained using the same training dataset and tested on the same testing dataset that was used for DeepLoc. To further reduce the bias resulting from inappropriate implementation or parameter configuration, we directly quote the performance reported in the DeepLoc paper [103] and show the prediction results in Table 4.6.

As shown in Table 4.6, MULocDeep has comparable performance in terms of prediction accuracy (each method has 5 out of 10 classes better than the other method). One reason is that our method used a much smaller database for PSSM profile generation (see Methods) than DeepLoc. A benefit of that is that MULocDeep is much faster than DeepLoc. It takes approximately 4.2 seconds to run MULocDeep per protein, which is a prediction speed more suitable for online usage than DeepLoc, which takes approximately 30 seconds per protein.

Table 4.6. Comparison of Method Effectiveness between MULocDeep and DeepLoc

\begin{tabular}{|l|l|l|}
\hline Localization & MCC from MULocDeep & MCC from DeepLoc \\
\hline Nucleus & 0.725 & $\mathbf{0 . 7 8 4}$ \\
\hline Cytoplasm & 0.549 & $\mathbf{0 . 6 0 8}$ \\
\hline Extracellular & 0.896 & $\mathbf{0 . 9 0 7}$ \\
\hline Mitochondrion & $\mathbf{0 . 8 2 3}$ & 0.812 \\
\hline Cell membrane & 0.696 & $\mathbf{0 . 7 3 2}$ \\
\hline Endoplasmic reticulum & 0.602 & $\mathbf{0 . 6 5 4}$ \\
\hline Plastid & $\mathbf{0 . 9 0 1}$ & 0.883 \\
\hline Golgi apparatus & $\mathbf{0 . 4 6 4}$ & 0.414 \\
\hline Lysosome & $\mathbf{0 . 2 0 8}$ & 0.194 \\
\hline Peroxisome & $\mathbf{0 . 4 1 2}$ & 0.321 \\
\hline
\end{tabular}

The comparison is at the subcellular level. Both methods were trained using the training samples in the DeepLoc dataset and tested using the testing samples in the DeepLoc dataset. 


\subsection{Attention weight visualization}

Before presenting the protein localization mechanism analysis based on attention weight, we introduce the methods we used for attention weight visualization. To visualize the attention for one protein, the average of the protein's attention matrix was calculated along the dimension of "heads", which is 41 in our model. A 1000-long vector is left and ready for visualization.

To visualize the attention of a group of proteins that belong to the same category, different methods are applied based on the following two scenarios:

1. For analyzing attention at termini, we simply align the first 50 or the last 50 amino acids one by one from left to right or right to left depending on if it is $\mathrm{N}$ terminus or C-terminus. For each position, the frequency of amino acids and the weight of attention (average along with the number of "heads" and proteins) are obtained. Then we used the R package "ggseqlogo" [118] to visualize the attention.

2. For analyzing attention in the middle of proteins, we kept the entire protein sequence and ranked the amino acid in it based on their attention weights. We selected the top 5 amino acids, each of them combining the surrounding 20 amino acids (10 window size at each side) to form a segment. A final segment was obtained by concatenating all segments by a string of " $\mathrm{X}$ " with the same length of window size. All segments belonging to the same class were analysed by the GLAM2 [119], a tool in the MEME Suite 5.1.0 that can discover variable-length, gapped motifs. 


\subsection{Protein localization mechanism interpretation powered by attention}

MULocDeep can not only make accurate localization predictions but also indicate the contribution of each amino acid in localization and suggest localization motifs. This is achieved by attentive embedding through assigning higher weights to specific parts of a protein sequence. We assume that the regions with higher attention weights are more likely to contribute to the localization. When using a high resolution of attention, it is possible to predict sites and motifs relevant to protein localization. For example, the peptide cleavage site could be predicted directly from an amino acid level attention [55]. Our method provides interpretable results for all the 44 types of suborganelle localizations as far as 500 amino acids from each terminus.

Firstly, we used several proteins with known localization signals as cases to demonstrate the ability of attention weights for indicating the contribution of each amino acid in localization. The proteins are: SV40 large T antigen (P03070) located at the nucleus, with the known signal motif "PKKKRKV" in the middle of the protein sequence; lactalbumin (P09462) located at the secreted pathway, with a known signal peptide "MMSFVSLLLVGILFWATEAEQLTKCEVFQ" at the N-terminus; and COX4 (P04037) located in the mitochondrial inner membrane, with the known transit peptide "MLSLRQSIRFFKPATRTLCSSRYLL" at the N-terminus [120-122]. When using these proteins as input, MULocDeep predicted the localization correctly for all three proteins. We also obtained the attention weights for each protein along the sequence, as shown in Figure 4.1, indicating that the high attention regions match the corresponding known signal motifs of the proteins. 

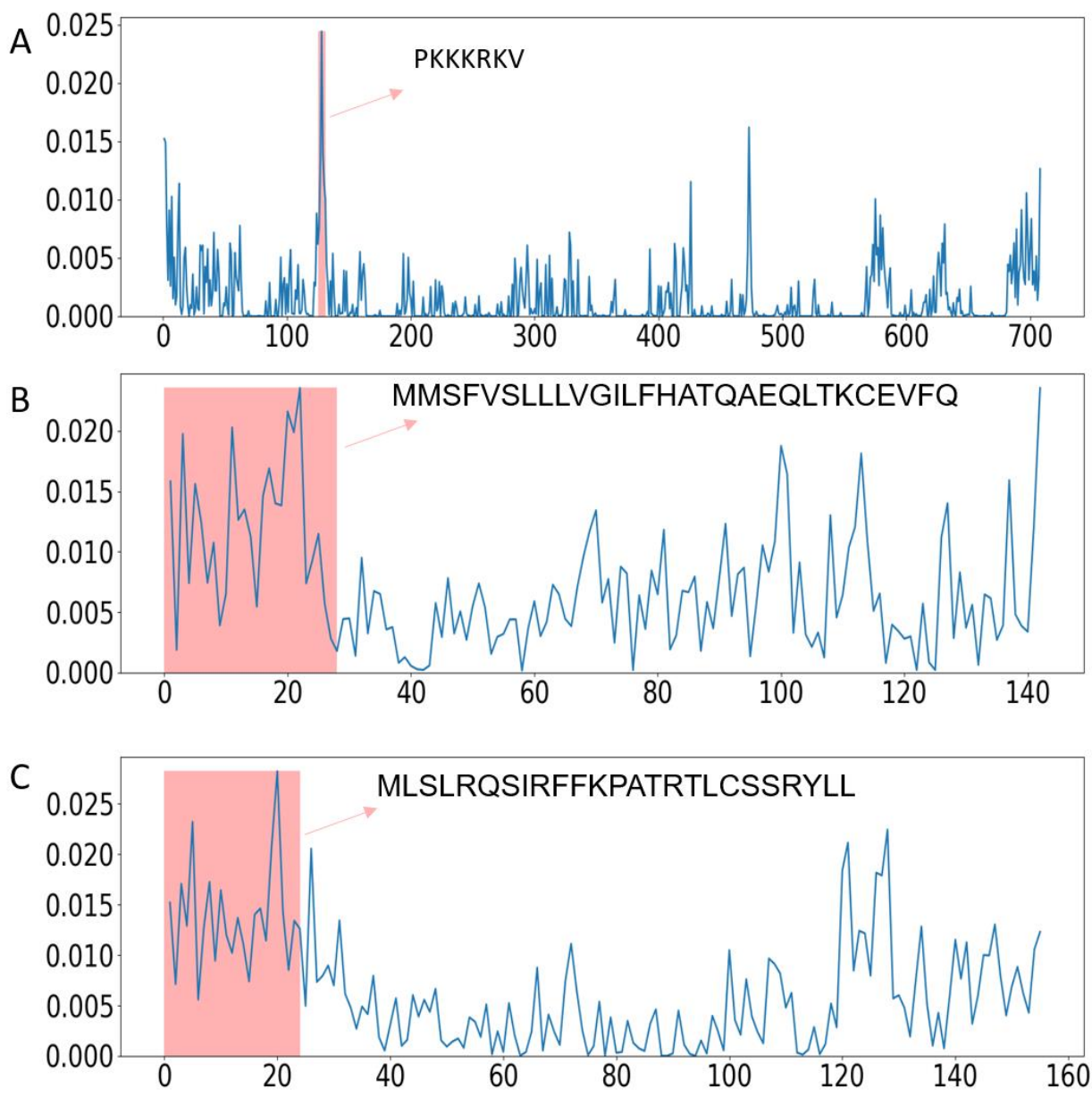

Figure 4.1. Visualization of attention weights for (A) SV40 large T antigen (P03070), (B) lactalbumin (P09462), and (C) cytochrome oxidase subunit 4 (P04037). The $\mathrm{x}$-axis presents the sequence position from the $\mathrm{N}$-terminus to the $\mathrm{C}$-terminus, and the $\mathrm{y}$-axis presents the value of attention weights. The region of the known sorting motif is highlighted in peach and labelled with the sequence of known localization signals.

Next, we investigated the attention weights in terms of groups of proteins from the same subcellular compartments and the same suborganelle compartments. Firstly, we visualized the attention weights of proteins from ten subcellular compartments (Figures 4.2 to 4.4). Among them, the localization of proteins in extracellular, mitochondrial, plastid, and thylakoid lumen (Figure 4.2) are believed to be controlled by signal peptides near the protein N-terminus [55]. Comparing to other localizations (Figures 4.3 and 4.4), 


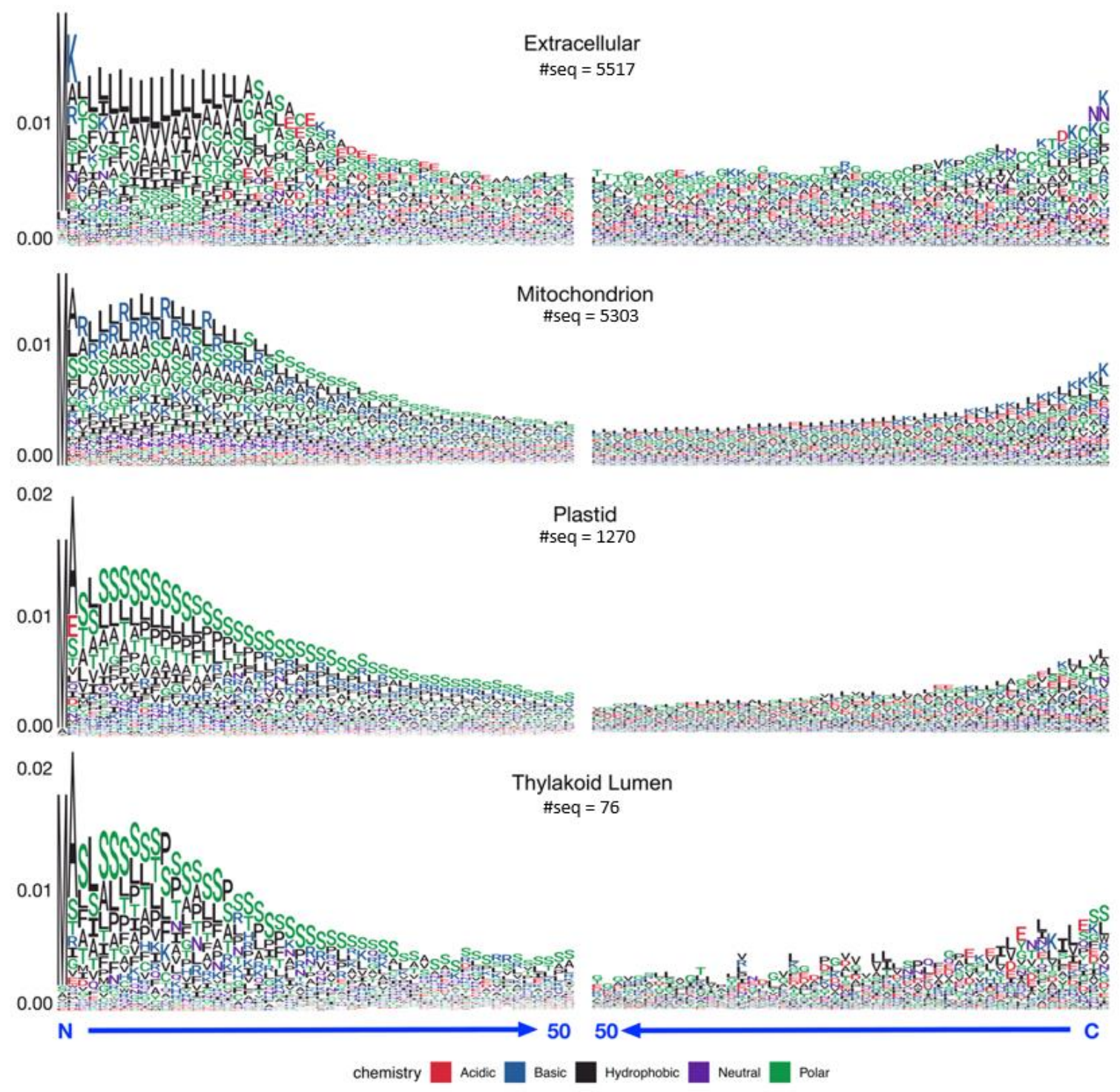

Figure 4.2. The attention weights at the $\mathrm{N}$-terminus (left column) and the $\mathrm{C}$-terminus (right column) for proteins localized in the extracellular, mitochondrion, plastid, and thylakoid lumen.

the signals near the N-termini of proteins in Figure 4.2 have higher attention weights, more over-represented amino acid patterns, and maintain at high levels for longer sequence segments. Our result is consistent with TargetP in detecting the N-terminal sorting signals using attention weights $[55,123]$. These $\mathrm{N}$-terminal sorting signals are often proteolytically removed at the cleavage cites after the protein reaches the final 


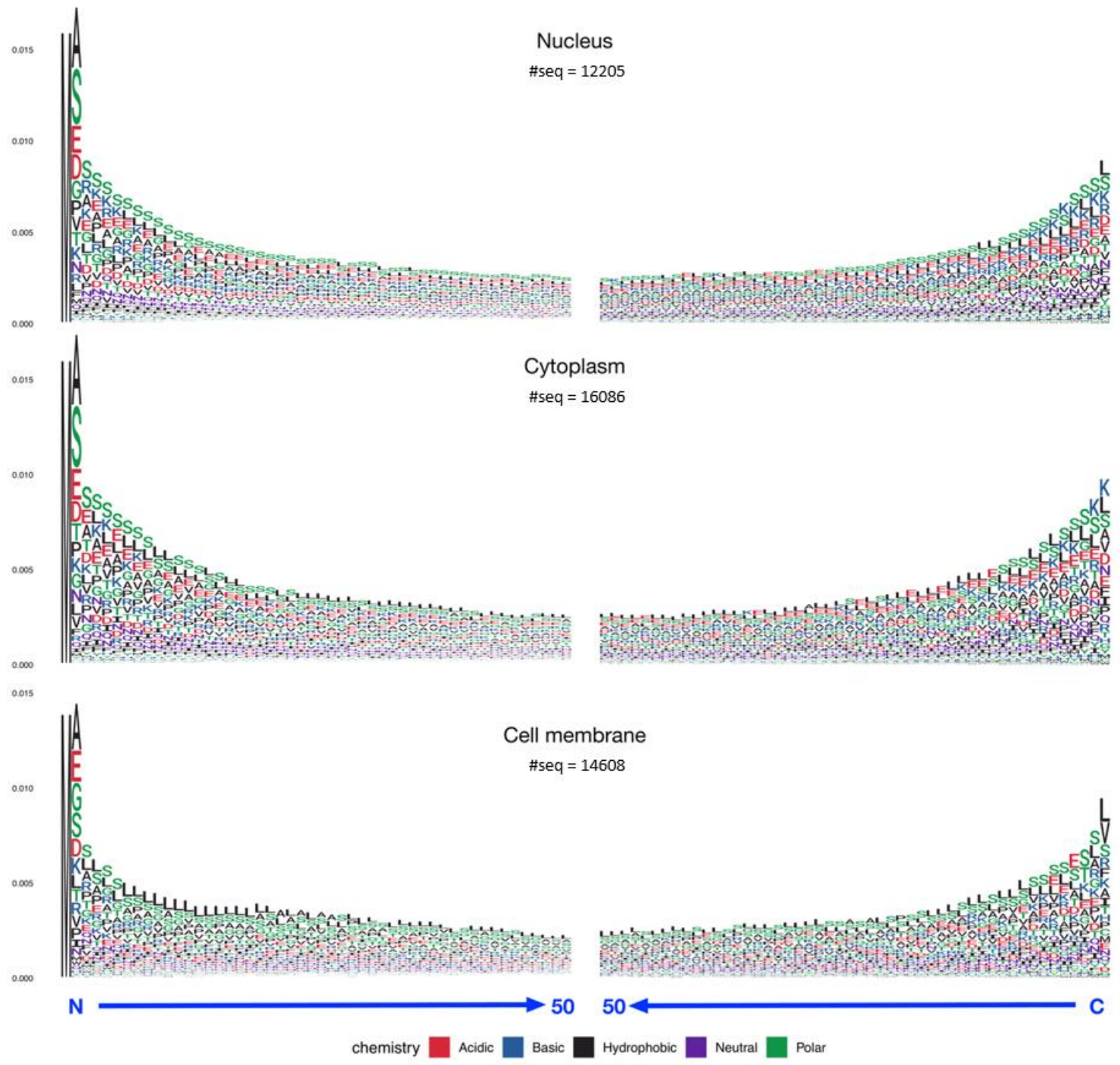

Figure 4.3. The attention weights at the $\mathrm{N}$-terminus (left column) and the $\mathrm{C}$-terminus (right column) for proteins localized at the nucleus, cytoplasm, and cell membrane.

destination. We then aligned the weighted sequences of these four types of proteins at the cleavage site. The cleavage site annotation was obtained from the UniProt database. The attention visualization result is shown in Figure 4.5. An immediate decrease in the attention weight is observed after the cleavage site for the proteins from all four 


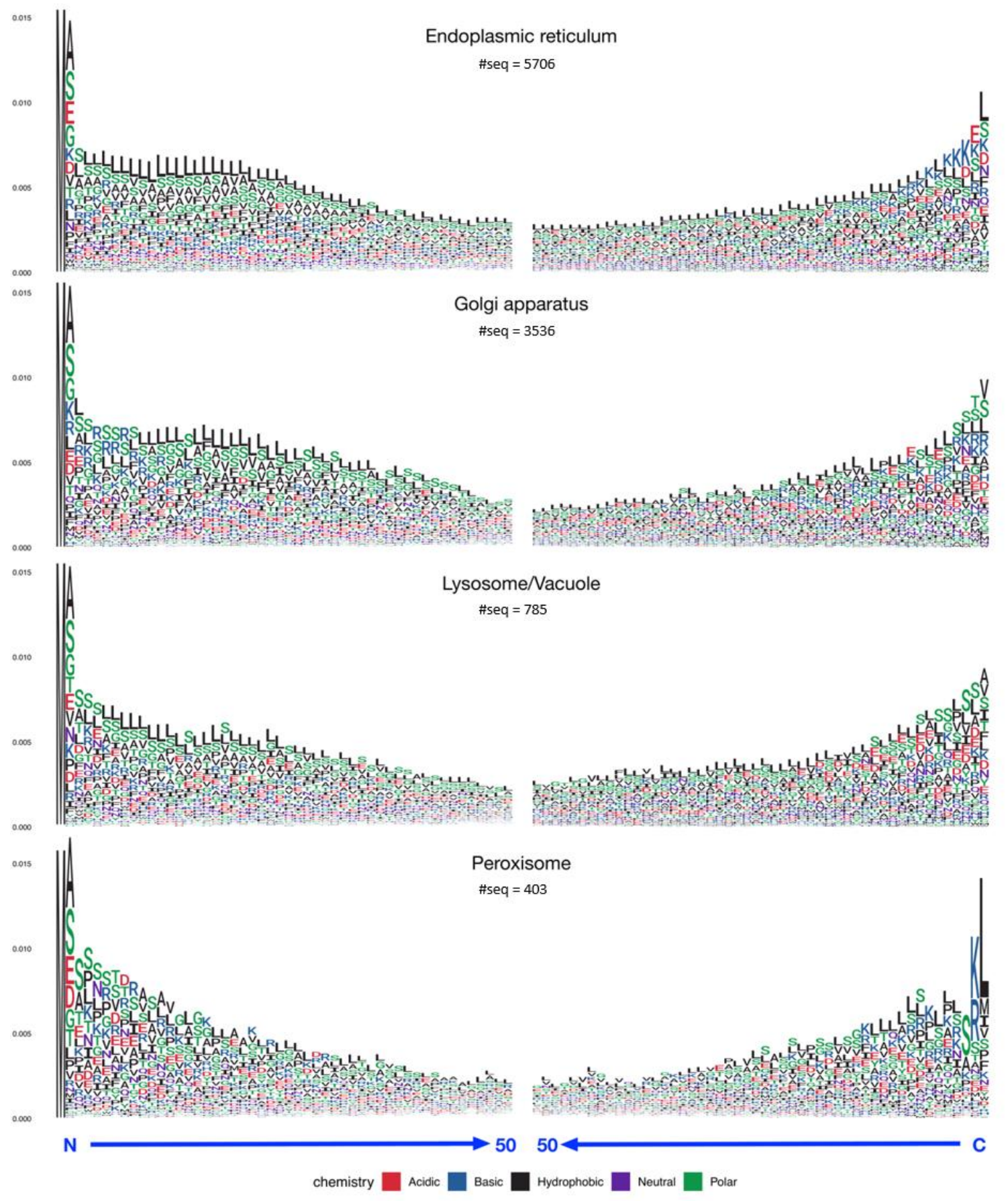

Figure 4.4. The attention weights at the N-terminus (left column) and the C-terminus (right column) for proteins localized at the endoplasmic reticulum, Golgi apparatus, lysosome, and peroxisome.

subcellular localizations. This indicates that the high attention weights near the $\mathrm{N}$ terminus are mostly contributed by signal peptides and transit peptides. 
Looking at the attention weights at the termini of proteins in all ten subcellular localizations (Figures $4.2-4.4$ ), it is apparent that the attention weight increases towards the termini in all cases although more so at the C-terminus. We, therefore, wondered if there is a terminus attention bias introduced by the MULocDeep method. We did a control experiment to test for such a terminus bias on the proteins. For each localization, we randomly shuffled the order of amino acids for each protein sequence. Then we

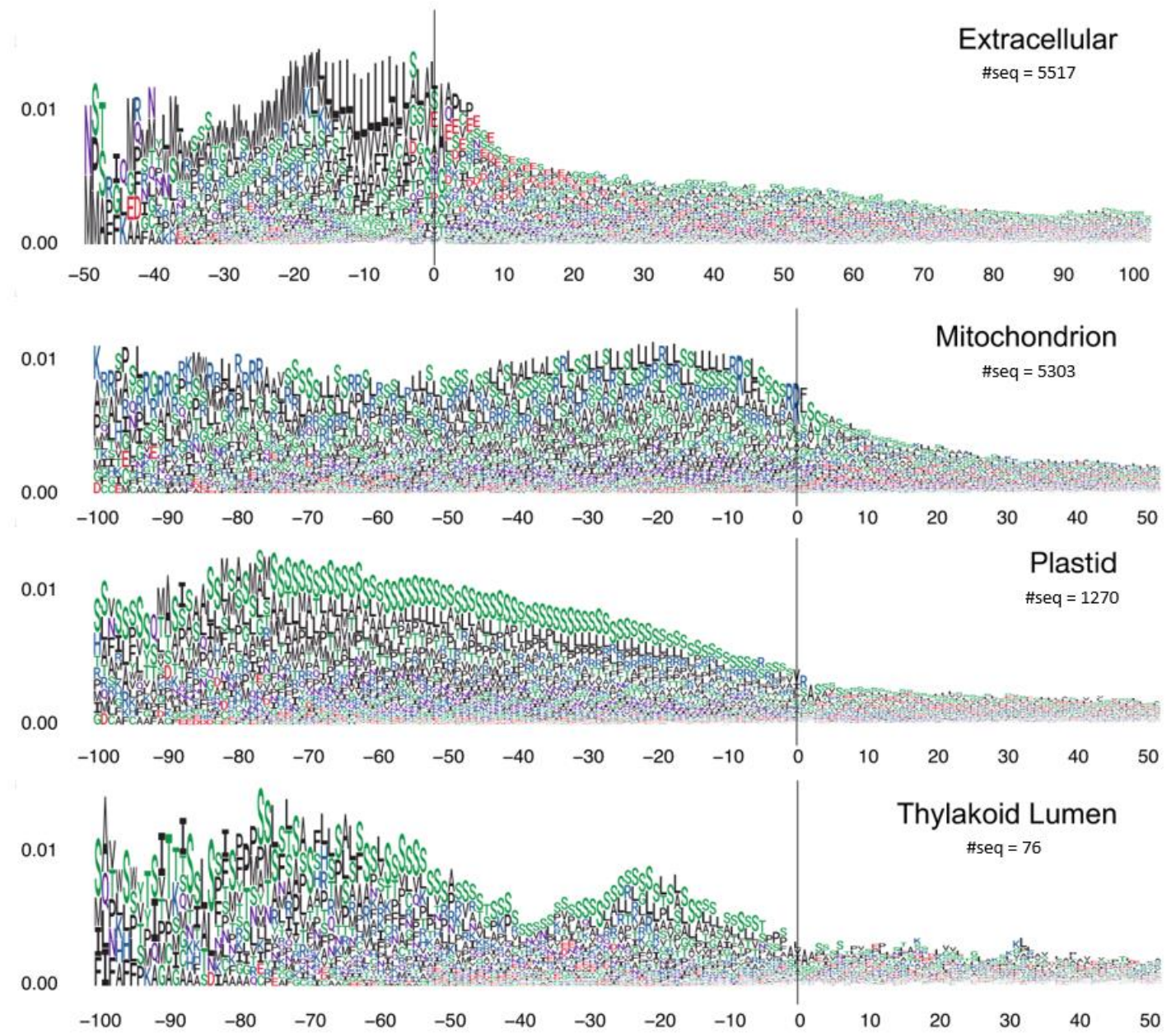

Figure 4.5. The attention weight vs. sequence position by aligned $\mathrm{N}$-terminal sequences at the cleavage site for proteins localized at extracellular, mitochondrion, plastid and thylakoid lumen. The vertical lines indicate the cleavage sites. For extracellular proteins, the range covers 50 AAs before the cleavage cite and 100 AAs after the cleavage cite. For the other three classes of proteins, the range covers 100 AAs before the cleavage cite and 50 AAs after the cleavage cite. The number of protein sequences in each class is shown. 
plotted the attention weights aligned at the termini for these four localizations and indeed found some terminal bias (Figure 4.6). We can use this to distinguish true and falsepositive signals, which all give high attention weights near the termini. As shown in Figure 4.6, false-positive signals are characterized by a gradual decrease from the terminus and the absence of dominant amino acids at each residue position. In contrast,

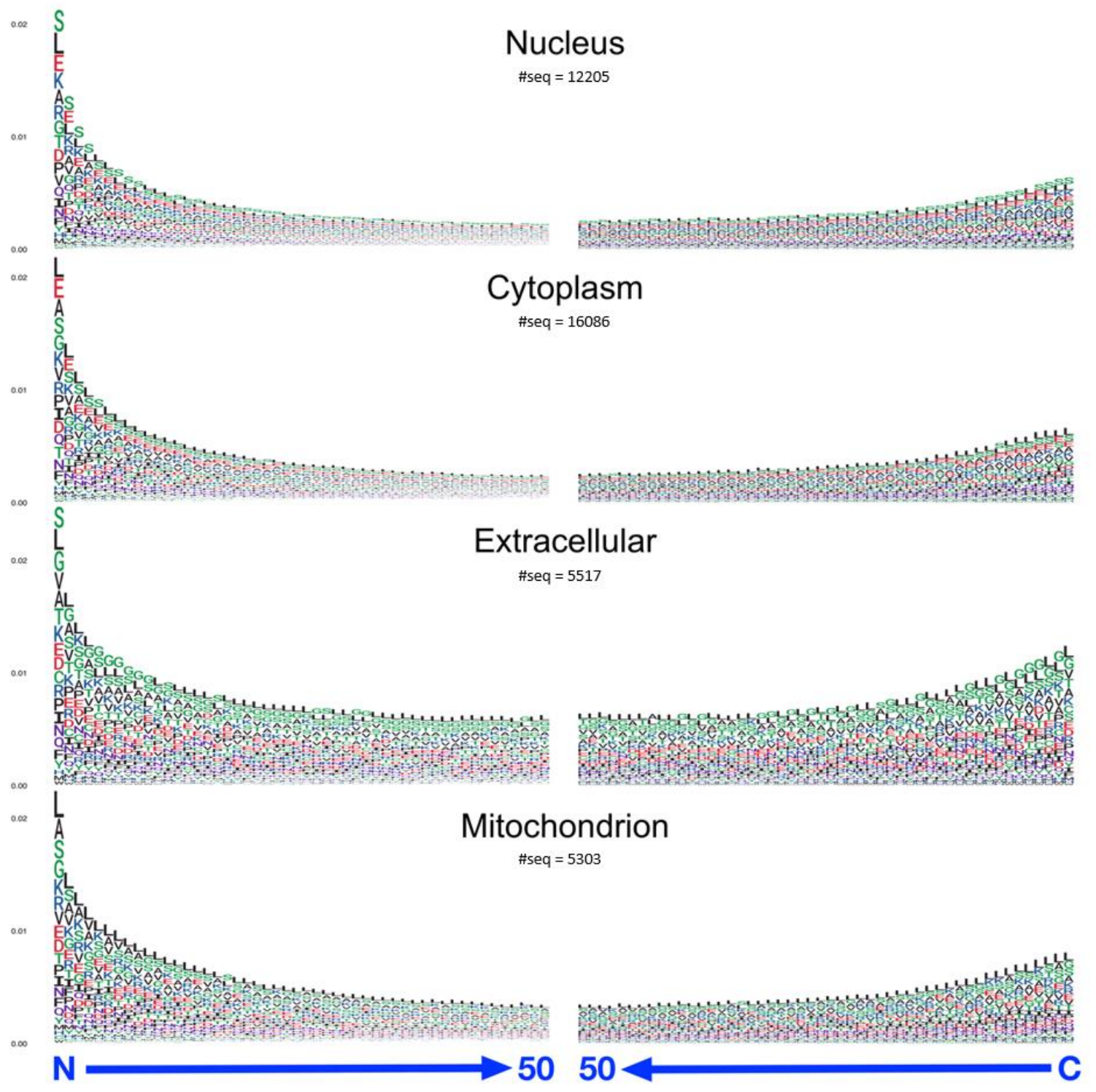

Figure 4.6. The attention weights at the $\mathrm{N}$-terminus (left column) and the C-terminus (right column) for proteins of randomly shuffled sequences in the nucleus, cytoplasm, extracellular, and mitochondrion. 
the features of true signals are: amino acids in each position are more conserved, the high attention signal lasts relatively longer and sometimes the highest attention weight appears as a 'bump' away from the termini (in all four localizations shown in Figure 4.2). Hence, although the terminus attention bias exists, the attention weights in MULocDeep may add values to illustrate biologically significant signals. We then divided the attention weights from the real protein sequences of the four protein classes in Figure 4.6 by the attention weights from the randomly shuffled protein sequences. The result is shown in Figure 4.7. For protein classes that do not have an N-terminal signal (nucleus, cytoplasm), their attention weights are similar to the random one and the attention ratio is near 1 . While for

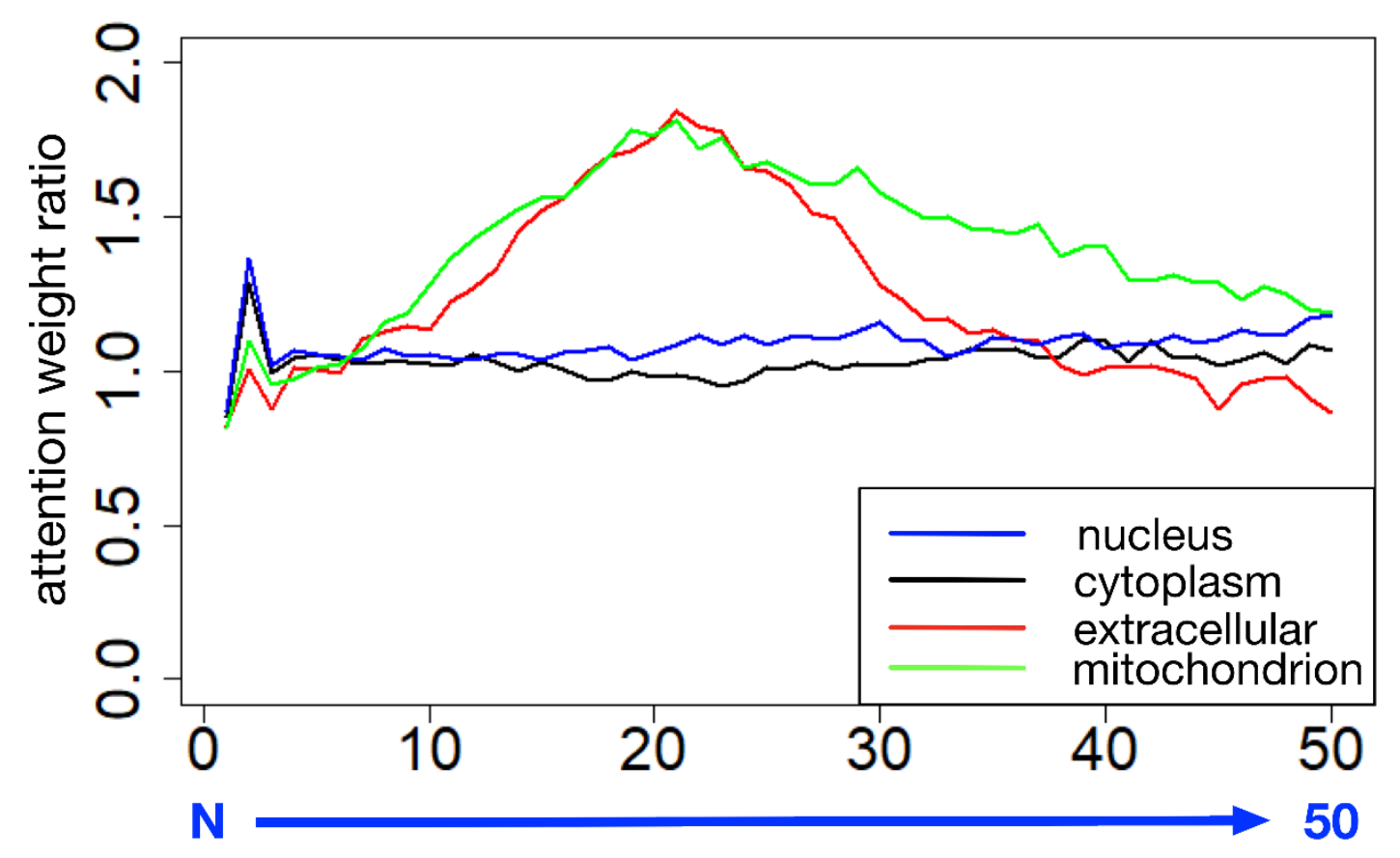

Figure 4.7. The ratios of $\mathrm{N}$-terminal attention weights from real protein sequences to $\mathrm{N}$-terminal attention weights from randomly shuffled protein sequences in the same protein class. 
the protein classes that are known to have an $\mathrm{N}$-terminal signal peptide, e.g., extracellular and mitochondrion, there is a region that the ratio is much higher than 1.

Since the attention weights of a subcellular localization reflect the average of attention weights of its suborganellar localizations, there still could be suborganellar
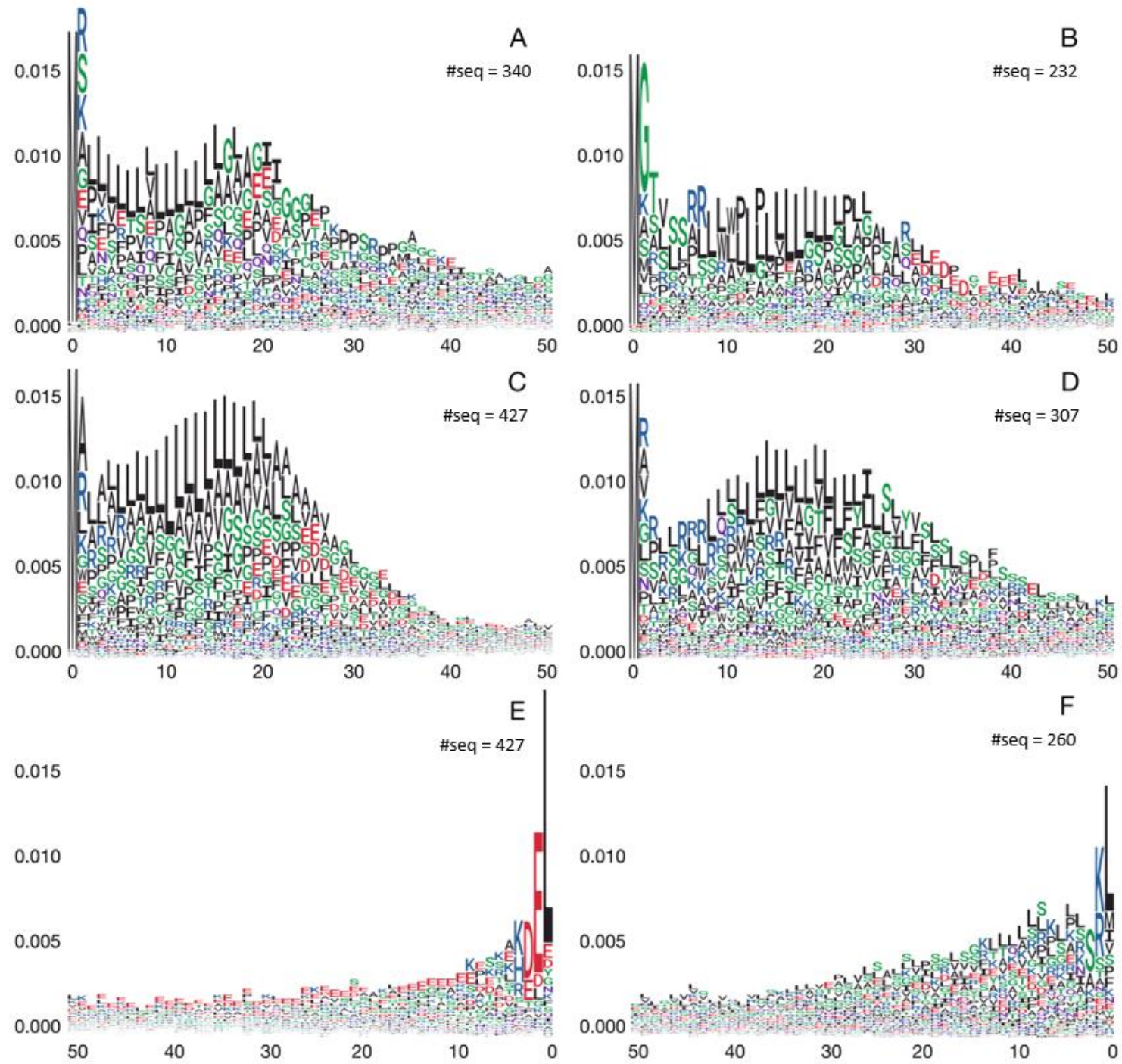

Figure 4.8. Attention weights of 50 amino acids vs. sequence position near the termini at the sub-organellar level, which suggests potential localization signals. (A) N-terminus of cytoplasmic granule. (B) N-terminus of cell surface. (C) N-terminus of endoplasmic reticulum lumen. (D) N-terminus of Golgi apparatus and Golgi stack membrane. (E) C-terminus of endoplasmic reticulum lumen. (F) C-terminus of peroxisome proteins. 
localizations that have strong $\mathrm{N}$-terminal or C-terminal sorting signals even though the subcellular sorting signal is not obvious. We, therefore, show the attention weights of proteins at the suborganellar localizations in nucleus, cytoplasm, cell membrane, endoplasmic reticulum, Golgi apparatus, lysosome, and peroxisome (Appendix B). We found several suborganelle localizations with strong signals near the termini. These signals are shown in Figure 4.8, including N-terminal signal peptides in proteins from cytoplasmic granule (Figure 4.8A), cell surface (Figure 4.8B) and endoplasmic reticulum lumen (Figure 4.8C), and perhaps also in Golgi apparatus membrane and Golgi stack membrane (Figure 4.8D), which all resemble the endoplasmic reticulum signal peptide observed for extracellular proteins in Figures 4.2 and 4.4. A C-terminal KDEL/HDEL signal in the endoplasmic reticulum lumen proteins (Figure 4.8E), a C-terminal SRL/SKL/SRM signal for the peroxisome (Figure 4.8F), and a less clear sequence for the peroxisome membrane (Figure A.10) $[124,125]$ are observed.

Besides the signals near the termini, we also analyzed the attention weights in the middle. We aligned sequences in the same way as we did at the termini, but no signal was found at all. A likely reason is that the signals in the middle do not appear in the same position for different proteins. Thus, the sorting signal in the middle of protein sequences was analyzed and visualized with the help of the GLAM2 tool [119] in the MEME Suite, which can discover over-represented, position-independent motifs in protein sequences (see Methods for details). A well-known internal signal is the nuclear localization signal (NLS). The visualization in Figure 5 using nuclear proteins was obtained by setting the 


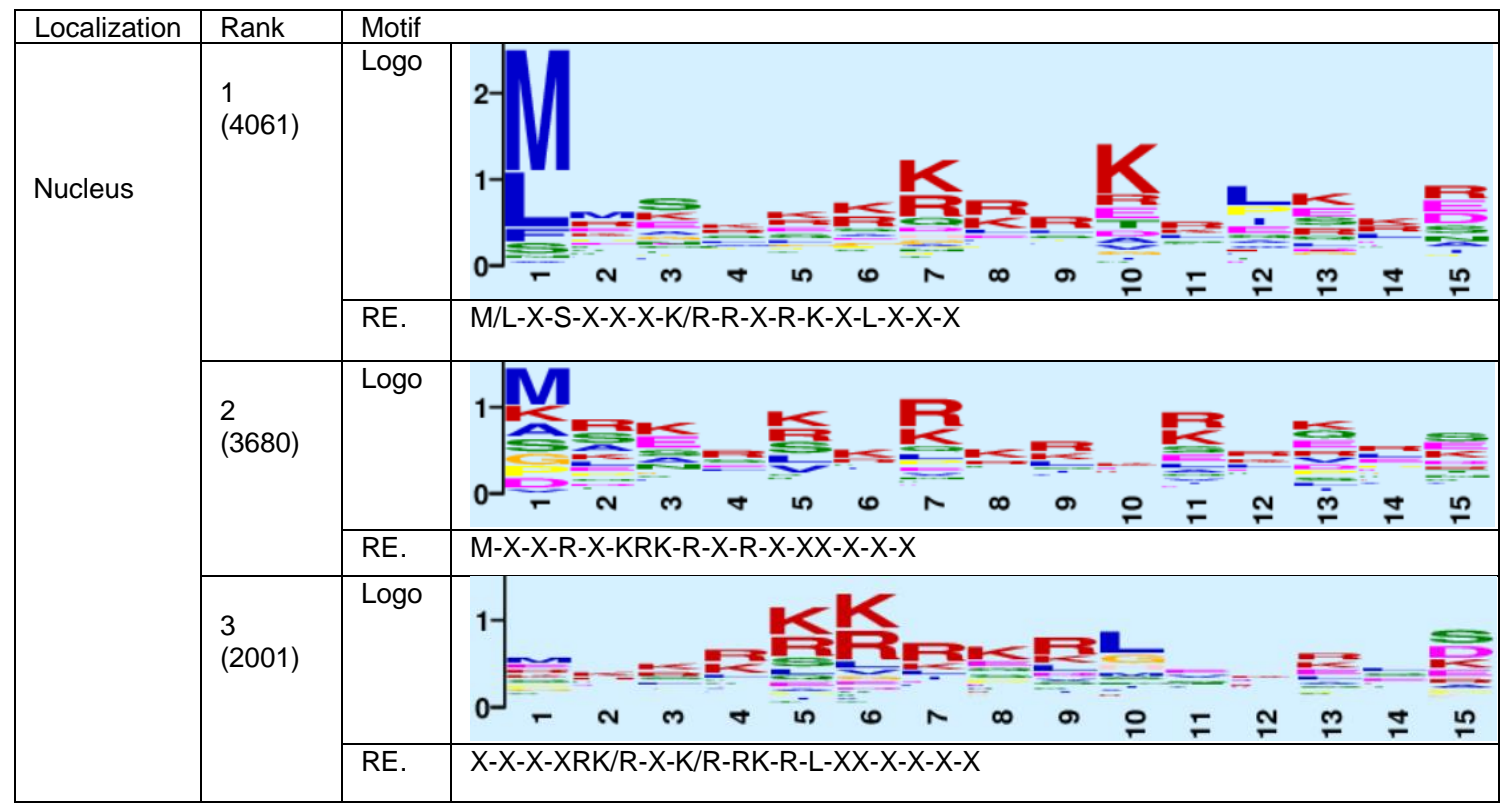

Figure 4.9. The top three GLAM2 results for segments from nucleus proteins. For each result, its rank, score, sequence logo and the regular expression (RE) of the motif are given.

"initial columns" (initial number of aligned columns in the motif) equal to 15 , the "maximum columns" (upper bound on the number of aligned columns in the motif) equal to 30 while other parameters remain the default in the GLAM2 online tool. The classical NLS pattern includes stretches of 4-5 Lys or Arg (e.g. KKKK) [120], which was readily recognized by GLAM2 (Figure 4.9).

We carried out similar GLAM2 analyses on proteins from six more subcellular localizations (Appendix C) and found a number of well-defined internal signals where the signal found in the cytoplasm deserves special mention (Figure B.1, motif rank 2) - A L/I/VxxxxxxL/V/I/F motif, which is known to be a nuclear export signal [126]. 


\section{CHAPTER 5. MULOCDEEP WEBSERVER}

Most biology researchers lack sufficient computational skills and prefer to use web servers or API tools to lessen the burden of data analysis. As a result, there is a gap between method development and the utilization by the people who need them the most. Our webserver provides a platform for predicting, analyzing, visualizing, and giving hypotheses to the sequence-based localization mechanisms for biologists and bioinformaticians. In this chapter, we will introduce the webserver of MULocDeep whose architecture is shown in Figure 5. Specifically, we will describe the front and back end of the webserver. We will also present a use case of using the webserver. The webserver can be accessed at https://www.mu-loc.org/.

\subsection{MULocDeep webserver architecture}

\subsubsection{Backend of the webserver}

The backend of the webserver uses Express, which is a minimal and flexible Node.js web application framework that provides a robust set of features for web and mobile applications. The main task of backend is to perform business logic and communicate with the database (MongoDB is our case). The business logic layer will cover all the analyses that can be performed by MULocDeep, such as protein localization prediction, and localization mechanism interpretation. The processing and verification of input data from users will also be performed in backend. 


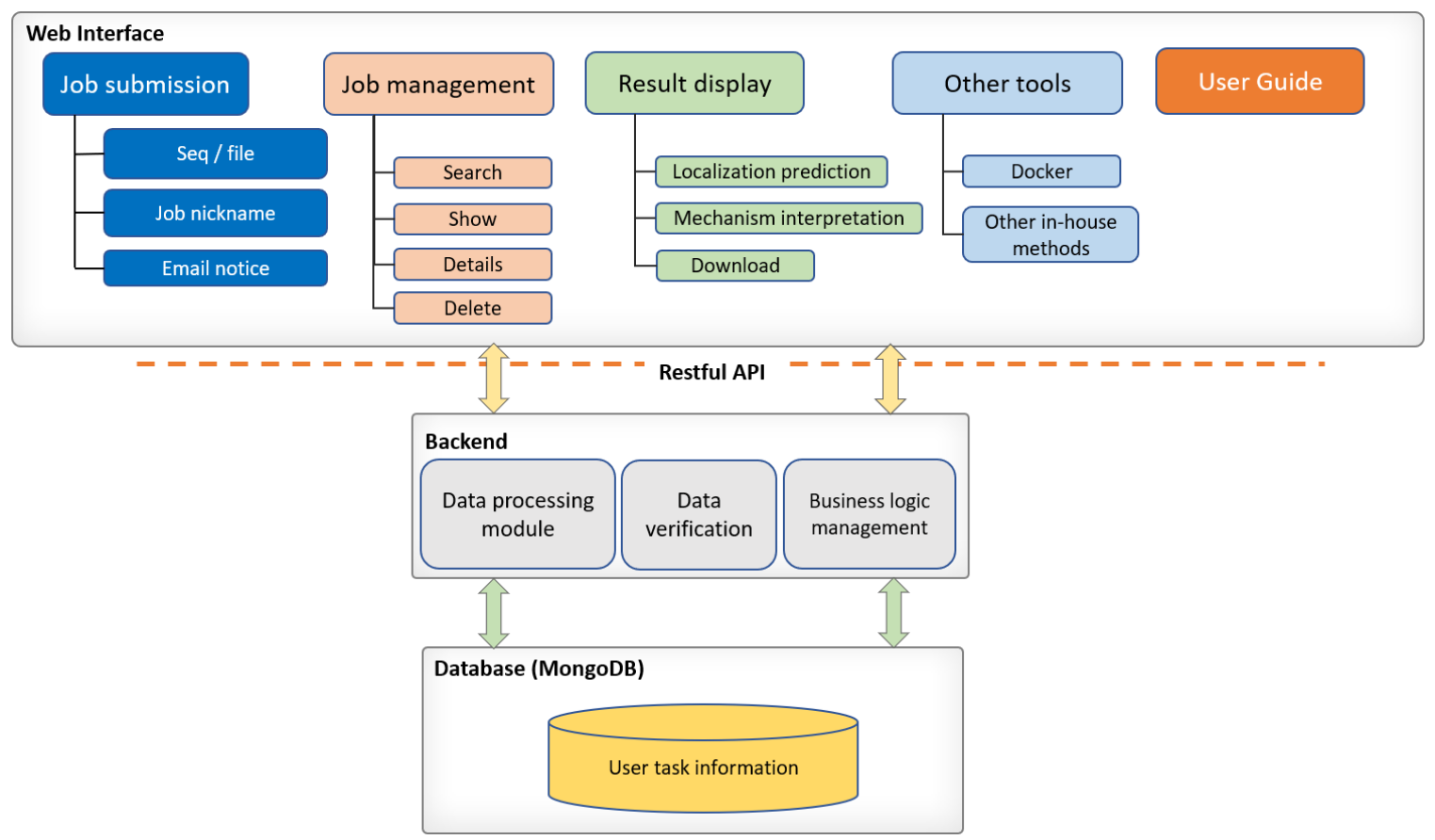

Figure 5.1. The architecture of the MULocDeep webserver.

\subsubsection{Frontend interface of the webserver}

The frontend will be communicate with the backend through the Representational State Transfer (REST) API $[127,128]$. The interface of the webserver is friendly, every page comes with a "help" button, which explains how the specific page works. It mainly contains the following layouts:

1. The job submission layout (main page) as shown in Figure 5.2 (A). Users can submit their job through pasting the protein sequences or uploading a file containing the protein sequences. Optionally, users can make a nickname for their job or providing an email address. The nickname is for searching the job.

Besides through nickname, jobs can also be searched by automatically generated 


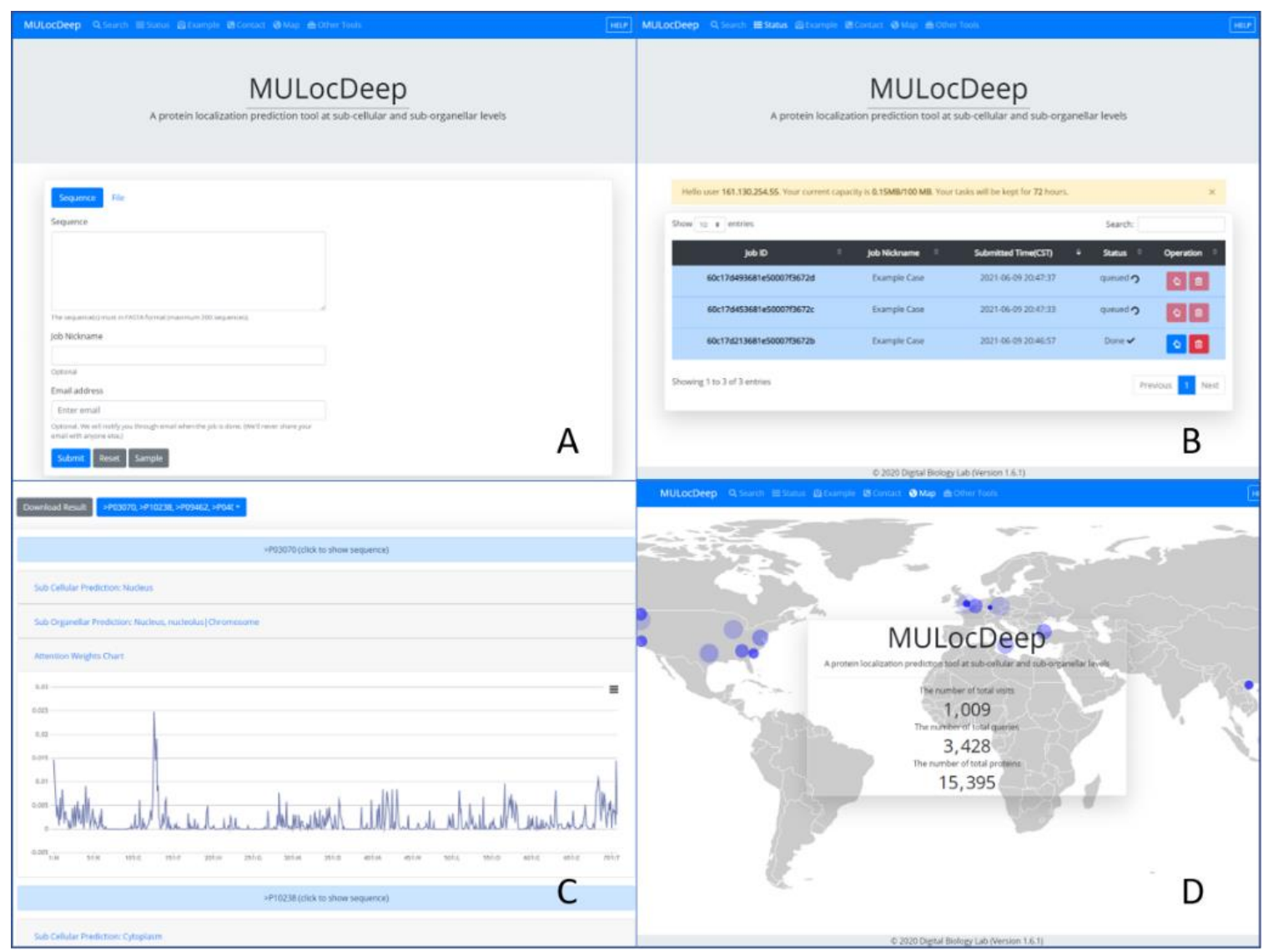

Figure 5.2. MULocDeep webserver screenshots. (A) job submission page. (B) job management page. (C) result presentation page. (D) webserver usage distribution and statistics.

job IDs or the sequences in the jobs. The email is for noticing once the job is done. After clicking the submit button, the backend of the webserver will first check the legality of the input data. The jobs that pass the verification will be put in the execution queue.

2. The job management layout as shown in Figure 5.2 (B). This is where users can manage their submitted jobs. Each user has his own workspace which is $100 \mathrm{MB}$. The status of the jobs is shown in this page, such as "queued" or "done". For the jobs that are "done", users can see the results or deleting them by clicking the 
corresponding buttons. The webserver will keep the results of the jobs for 72 hours before deleting them automatically.

3. The result displaying layout as shown in Figure 5.2 (C). This is where users can view the prediction results. We generate three kinds of prediction for each protein. They are subcellular localization prediction, suborganellar localization prediction, and attention weight visualization. Both levels of localization prediction are shown by bar plots. The attention weight visualization is shown by a interactive line chart, users can zoom in and out to check the potential localization contribution by each amino acid. The localization prediction results can be downloaded as tab separated file. And the attention weight can also be downloaded as image of PNG or JPEG format.

So far, the server has received $1000+$ visits, $3000+$ queries and $15,000+$ of proteins have been analyzed (Figure 5.2 (D)). It is becoming a valuable resource for protein localization researchers.

\subsection{Application of MULocDeep webserver to the human proteome}

We applied MULocDeep in the human proteome and drew pie charts of statistics in Figure 5.3. The human proteome data is collected from the UniProt database release 2020_04 [33]. Totally, 20,375 reviewed human proteins were collected. The green part in the left pie chart in Figure 6 containing proteins with experimentally verified localization annotations (ECO: 0000269) is further divided based on if the experimental evidence is at the subcellular level, suborganellar level, or both. Since subcellular localization can be inferred from suborganellar annotation, one can consider all the proteins in the green part 
to have subcellular level experimental evidence. For the proteins without experimental evidence or without localization annotation at all (proteins in the orange part in the left pie chart), we combined them and applied MULocDeep. The right pie chart is drawn based on the prediction results. More than half the proteins are predicted to be localized in the nucleus and cytoplasm. This result is consistent with the conclusion in Thul et al [29], which was obtained by mapping 12,003 human proteins at a single-cell level using immunofluorescence microscopy. Furthermore, according to Figure 5.3, the number of human mitochondrial proteins is 1347 (combining the proteins with experimental evidence and the proteins predicted by MULocDeep). This number matches the conclusion in Calvo et al [129], which estimated 1100-1400 distinct proteins in the human mitochondrial proteome. Thus, the prediction results in Figure 6 reflect the true localization distribution in human and demonstrates the ability of MULocDeep for proteome-wide annotation.

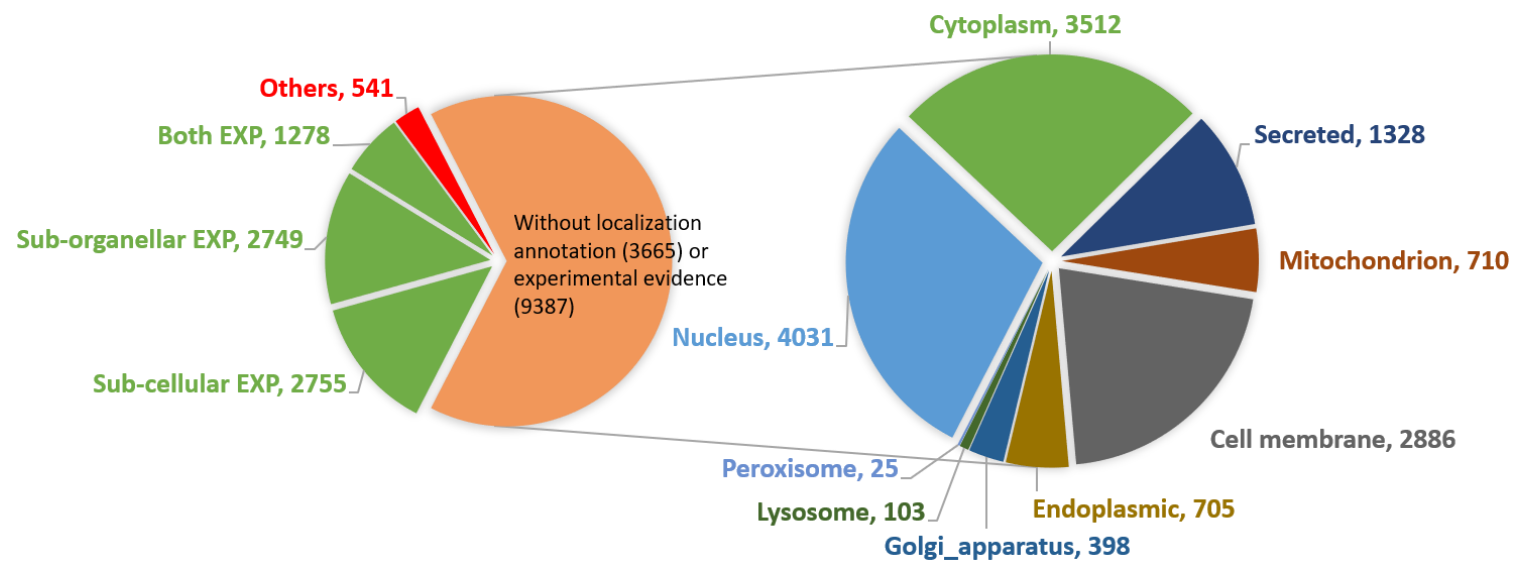

Figure 5.3. The human proteome localization pie chart. The left pie chart is based on the protein localization annotation, including with or without annotation, and with or without experimental evidence (ECO: 0000269). The green part in the left pie chart are proteins with experimentally verified localization annotations (ECO: 0000269). "Others" include those proteins contain annotations not in any of the 10 major sub-cellular or 44 sub-organellar localization annotations in MULocDeep, or have different localizations for different protein isoforms. The right pie chart is based on the distribution of prediction for the proteins in the orange part in the left pie chart. 


\section{CHAPTER 6. DISCUSSION AND CONCLUSION}

\subsection{Discussion}

\subsubsection{Noticed issues in the MULocDeep project}

When compare the localization prediction performance of MULocDeep with other methods, the DeepLoc dataset is one of the benchmark datasets we chose. Note that even though the DeepLoc dataset was claimed to be split the training and testing using PSICD-HIT with $30 \%$ sequence identity, we found that $44 \%$ of testing samples can find a sequence in training set that has a sequence identity above $30 \%$. Many samples in testing have very high sequence identity with samples in training: $2.2 \%$ have sequence identity above $80 \% ; 0.79 \%$ have sequence identity above $90 \%$. This is, to some extent, caused by the heuristic nature of the algorithm of PSI-CD-HIT. However, this make the DeepLoc dataset not a suitable dataset for method generalization evaluation. Our UniLoc dataset consists of UniLoc-train and UniLoc-test, as well as UniLoc-train-40nr where the sequences in UniLoc-train were removed if they have a sequence identity above $40 \%$ with a sequence in UniLoc-test using blastp (see Methods section). Thus, even though the identity threshold we used in blastp is higher (40\% versus $30 \%$ in deeploc using PSI-CDHIT), the actual resulting dataset is much more rigorous in terms of non-redundancy.

To analyze the attention in the middle of proteins, we used the GLAM2 tool in the MEME suite. Note that the analysis by GLAM2 was influenced by the number of top amino acids and the window size we chose. The parameters of the GLAM2 itself could 
also affect the results. Changing the parameter configuration would result in different visualization results, but the general motif information remains the same.

\subsubsection{Other types of protein localization mechanisms}

Besides targeting peptides, protein localization is controlled by some other factors as we already mentioned in Introduction. Here we briefly introduce the mechanism for each of them.

\subsubsection{Protein trafficking machinery}

The trafficking machinery in cells control the transport of molecules across membranes of organelles. Take nuclear pore complex (NPC) as an example, it allows the transport of RNA and ribosomal proteins moving from nucleus to the cytoplasm and proteins, carbohydrates, signaling molecules and lipids moving into the nucleus. It is notable that the NPC can actively conduct 1000 translocations per complex per second. Although smaller molecules simply diffuse through the pores, larger molecules may be recognized by specific signal sequences and then be diffused with the help of nucleoporins into or out of the nucleus. It has been recently shown that these nucleoporins have specific evolutionary conserved features encoded in their sequences that provide insight into how they regulate the transport of molecules through the nuclear pore $[130,131]$.

Dysregulation of the protein trafficking machinery can have dramatic effects on general protein transport processes [132]. For example, the homozygous mutation R391H in the nucleoporin NUP155 has been shown to reduce nuclear envelope permeability and

affect the export of Hsp70 mRNA and import of HSP70 protein [133]. Mutations in other 
components of the NPC, such as the nucleoporin p62 protein and ALADIN (alacrima achalasia adrenal insufficiency neurologic disorder, officially known as AAAS) are thought to cause the neurodegenerative diseases infantile bilateral striatal necrosis and triple A syndrome, respectively $[134,135]$. Mutant ALADIN prevents nuclear entry of the DNA repair proteins aprataxin and DNA ligase I and, therefore, results in increased DNA damage and subsequent cell death caused by oxidative stress [135]. In a similar fashion, protein import into other organelles can be affected by mutations in the trafficking machinery. For instance, mutations in the peroxin gene PEX7, which encodes a peroxisomal import receptor that is responsible for the transport of several essential peroxisomal enzymes, have been found to cause the peroxisome biogenesis disorder rhizomelic chondrodysplasia punctata type 1 (RCDP1) [136].

\subsubsection{Protein interaction}

Another common method of adjusting protein localization is for a binding partner to carry the bound protein between compartments. By necessity, this form of regulation requires a pore that can handle multiple folded proteins, such as the nuclear pore, ciliary boundary and the peroxisomal pore, but is not applicable for a system that generally requires an unfolded protein, like the mitochondrial or ER translocons. This mechanism allows the localization of a protein to be controlled indirectly by regulating the localization and levels of its binding partner, which plays a very similar role to import receptors. An example of this mode of regulation is with the minichromosome maintenance proteins (MCM2-7) that forma core part of the replicative helicase and are critical for origin firing in DNA replication [137]. MCM2 and MCM3 both have weak NLSs, but when bound together they form a single strong NLS, while MCM3 has an 
NES. MCM4 through MCM7 do not contain any localization signals, and their localization depends entirely on binding to MCM2 and MCM3 [138, 139].

\subsubsection{Protein folding}

Some localization signals are not contained within the linear peptide sequence of a cargo protein, but are formed by the arrangement of amino acid residues on the surface of the protein. An advantage of such an arrangement is that conformational changes induced by allosteric events can disrupt or reform the localization signal transiently and in response to the state of the protein. A well-characterized example of a conformationsensitive NLS is found in the fatty acid transporter, fatty acid-binding protein 5 (FABP5). When its fatty acid ligand (e.g. linoleic acid and arachidonic acid) is bound, several basic residues on the surface of the protein align with one another. This alignment allows importin to bind to and escort FABP5 and its ligand into the nucleus for delivery to lipidresponsive nuclear receptors. However, when the ligand-binding pocket is emptied, those basic residues are shifted out of alignment, rendering the FABP5 surface unrecognizable to importins and preventing further active nuclear import [140].

\subsubsection{4 cell-type/tissue-specific localization}

Some proteins show their tissue specificity at the subcellular level. First of all, some particular subcellular compartments exist only in certain specific tissues. For instance, the sarcolemma is a unique subcellular compartment in muscle tissue. Moreover, the spatial distribution of the SCLs of the protein in a cell could be different from one tissue to another, which depends on the functions of the protein in the specific tissue. For example, glutamine synthetase (GS) is mitochondrial in liver cells and cytoplasmic in brain cells [141]. In the human tissue adrenal gland, pituitary gland, and pancreas, the absence of 
adracalin (ALADIN) in nuclear membrane causes human triple A syndrome [142]. Therefore, understanding the specific SCLs of human proteins in different tissues and organs of the human body would significantly increase our knowledge of human biology and disease.

\subsubsection{5 mRNA localization}

One last specific mechanism available to cells to control protein localization is to localize mRNA transcripts near the location where the protein is intended to function. As in the case of microtubule-mediated transport, this mode relies on increasing the local concentration of the protein where it is most likely to interact with import receptors, making transport more efficient. One protein that uses this pathway is metallothionein $1 \mathrm{~A}$ (MT1A), where the nuclear concentration of the protein depends directly on its mRNA localizing near the nucleus [143]. Some mitochondrial [144] and peroxisomal [145] transcripts are also directed in this manner. Localization of protein production is especially important for neurons, which often have extreme distances between the nucleus and sites of protein activity [146]. In our recent work [147], we have already did some mRNA localization predictions and mechanism interpretations.

\subsubsection{Future work}

The studies of the localization mechanisms mentioned above are appealing, however, a systematical framework for such research is missing. As machine learning is developing fast these days, new sophisticated machine learning methods have been continuously proposed, and make it possible to fill the gap in localization research by applicating them. Here we propose several topics for future studies. 


\subsubsection{Develop an interpretable end-to-end protein localization prediction framework by combining sequence and graph models.}

Our MULocDeep model has shown the potential of using attention mechanism on sequence model to improve protein localization prediction and identify potential targeting signals. Thus, we propose to develop a novel deep learning framework to further improve the prediction performance and, more importantly, to model and interpret sequence-based (targeting signals) and interaction-based (gene regulation or physical interactions) localization mechanisms. To achieve this, the framework will incorporate the features of the most advanced attention-based machine learning techniques in its two components, the sequence model and the graph model.

There are many deep learning structures for sequential input. For example, the recurrent neural network (RNN), Long Short-Term Memory (LSTM), Gated Recurrent Units (GRU), Transformer [148], and its most recent derived model ELECTRA [149]. One of our ongoing projects is trying to take advantage of pre-train model provided by ELECTRA.

PPI contains important information regarding protein localization since interacting proteins are more likely to be co-localized [150]. We can use the protein interaction information plus known localization annotation of some proteins to predict the localization for other proteins in the graph. Graph information could also help to model the protein localization mechanism related to molecular interactions and regulations besides the targeting signals. For these purposes, graph attention networks will be incorporated in our framework. 


\subsubsection{Extend the framework for cell-line-specific localization study using single-cell data}

The framework proposed above provides a baseline for general localization prediction and mechanism interpretation. When cell-line-specific data is available, we will fine-tune the baseline framework for better prediction performance and more biological insights. Specifically, the biological context-specific protein interaction network and localization annotations are required. These context-specific protein networks can be either already existed or created from other data types, for example, expression data. Then, a transfer-learning will be applied after replacing the general PPI with context-specific protein networks.

\subsubsection{Inferring the molecular and phenotypic impact of mislocalization related amino acid variants}

Protein mutations could result in abnormal cellular function through numerous mechanisms. One of them is by changing the protein localization. For example, the substitution R173C in the homeobox-containing gene SHOX prevents the transport of the SHOX-encoded protein to the nucleus and its subsequent function as a transcription activator, which causes idiopathic short stature, Leri-Well dyschondrosteosis and Langer mesomelic dysplasia [151]. Another example is Barttin, encoded by BSND, whose wildtype localizes to the plasma membrane. The mutant forms are retained in the ER and are involved in Bartter syndrome, a renal tubular alt-wasting disease [152].

To date, pathological and phenotypic mechanisms have been revealed for only a fraction of all known mutations. It is tedious, expensive and difficult to test experimentally the impact of mutations at the proteome scale. Numerous bioinformatic 
methods have been developed to predict and explain the consequences of either the mutations [153-155] or the mislocalizations [156-158]. Relatively few detailed investigations have described protein mislocalization due to disease-related mutations or introduced genetic alterations. Thus, it would be interesting to analyze the impact of protein mutation to phenotype from the mislocalization perspective.

\subsection{Conclusion}

In this paper, we present "MULocDeep", a deep learning method for protein localization prediction (shown in Figure 3.1). The core of the model is the bidirectional LSTM to handle protein sequence information and the multi-head self-attention to assign weights to each amino acid of a sequence for interpretation. Some methods [103] added CNN layers before LSTM layers and reported high performance but at the cost of resolution for residue-level interpretation in contribution to protein localization. Here, we discard CNN to pursue a residue-level interpretation resolution for more biological insight.

To maximize prediction accuracy, we used a Bayesian optimization method to determine the hyperparameters. However, our experiments showed that the performance was insensitive to the selection of hyperparameters (Table 3.4). This indicates that our model is robust. The selected configuration of hyperparameters remained the same for a variant model and the MULocDeep_tool, instead of optimizing a new set of hyperparameters with the specific data, which could have yielded slightly better results.

Evaluations of different studies were conducted from both perspectives of method and tool. Representative methods at both subcellular and suborganellar levels were 
compared with a variant MULocDeep model, which was trained and tested with their own data. We also provide several benchmark datasets with the experimentally extracted mitochondrial proteome from three species (Mito3 dataset) and a comprehensive dataset for 44 suborganelle protein localization from the UniProt database (UniLoc dataset). The UniLoc-train-40nr dataset is a non-redundant training set by removing proteins in UniLoc-train that have sequence identities above $40 \%$ against the UniLoc-test dataset. The Mito3-40nr is the non-redundant version derived by removing proteins that have sequence identities above $40 \%$ against the UniLoc-train-40nr dataset. These benchmark datasets were used for a comprehensive evaluation of localization classifiers at both subcellular and suborganellar levels (Table 4.3). The results indicate a superior performance and a good generalization ability of MULocDeep.

The attention weight mechanism in the MULocDeep model can detect sorting signals at the protein termini and in the middle of protein sequences. The interpretation was validated by matching the known transit peptides for proteins located in the nucleus, extracellular, mitochondria, plastid, and thylakoid lumen (Figure 4.2). Particularly, plastid and thylakoid lumen proteins have a high attention peak at position 2 from the $\mathrm{N}$ terminus, which is enriched in alanine. A region enriched in serine follows. The signal peptide in extracellular proteins apparently consists of a positively charged amino acid followed by a number of hydrophobic residues, a well-known endoplasmic reticulum localization signal (Figure 4.4) [159]. The mitochondrion transit peptides are enriched in arginine and leucine. For other subcellular (Figures 4.3 and 4.4) and suborganellar (Figures A.1-A.10) classes of proteins, we also found high attention regions near both Nand C-termini. Although most of them appear to be caused by terminus bias, several 
known signals were recognized; in particular, a well-known $\mathrm{N}$-terminal endoplasmic reticulum signal peptide recognized in extracellular proteins (Figure 4.2) was also seen in proteins from cytoplasmic granule (Figure 4.8A) and cell surface (Figure 4.8B), both of which pass through the endoplasmic reticulum on the way to those localizations [159]. This $\mathrm{N}$-terminal signal was particularly strong in proteins belonging to the endoplasmic reticulum lumen (Figure 4.8C), where it was seen together with a C-terminal KDEL/HDEL signal (Figure 4.8E), which is an endoplasmic reticulum retention signal [160-162]. We also found a clear C-terminal SRL/SKL/SRM signal in peroxisome proteins (Figure 4.8F), which is a targeting signal for proteins belonging to the peroxisome $[124,125]$.

To analyze the attention in the middle of proteins, we used the GLAM2 tool in the MEME suite, and we demonstrate the effectiveness of this approach by visualizing the attention of proteins located in the nucleus (Figure 4.9). We also found a clear $\mathrm{L} / \mathrm{I} / \mathrm{V} \operatorname{xxxxxL/V/I/F}$ motif in proteins belonging to the cytoplasm (Figure B.1), which is known to be a nuclear export signal [126]. For other localizations, where the mechanisms are unclear, we provide a list of attention visualizations in Figures B.2-B.6. They could be used as candidate motifs for these localizations.

There are limitations to the MULocDeep method, which lead to several future studies. With that said, we are still a long way from understanding protein localization fully. Given the complexities of decoding signal peptides or extracting features that can distinguish detailed levels of localizations, the interpretability of MUlocDeep makes it possible to generate hypotheses regarding protein sorting mechanisms. Some of them can be verified by current knowledge, making the rest worth exploring by combining 
computational prediction methods and experimental verification. By making the datasets, the software code, and the MULocDeep website publicly accessible, our study constitutes a major step toward understanding the protein localization mechanism. 


\section{APPENDIX A. ATTENTION WEIGHTS OF PROTEINS AT THE SUBORGANELLAR LOCALIZATIONS}

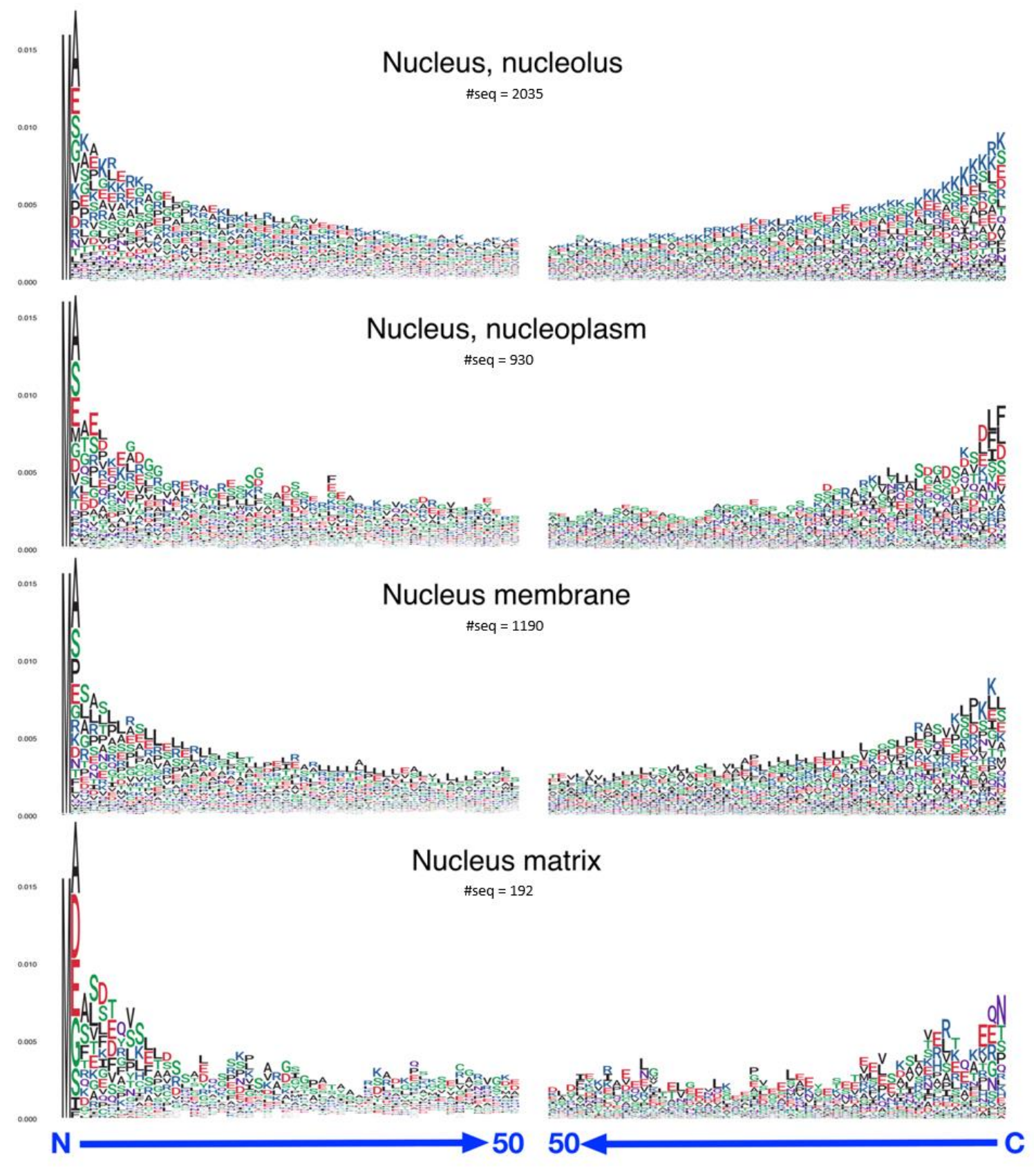

Figure A.1. The attention weights at the N-terminus (left column) and the C-terminus (right column) for protein localizations in the nucleus organelle. 


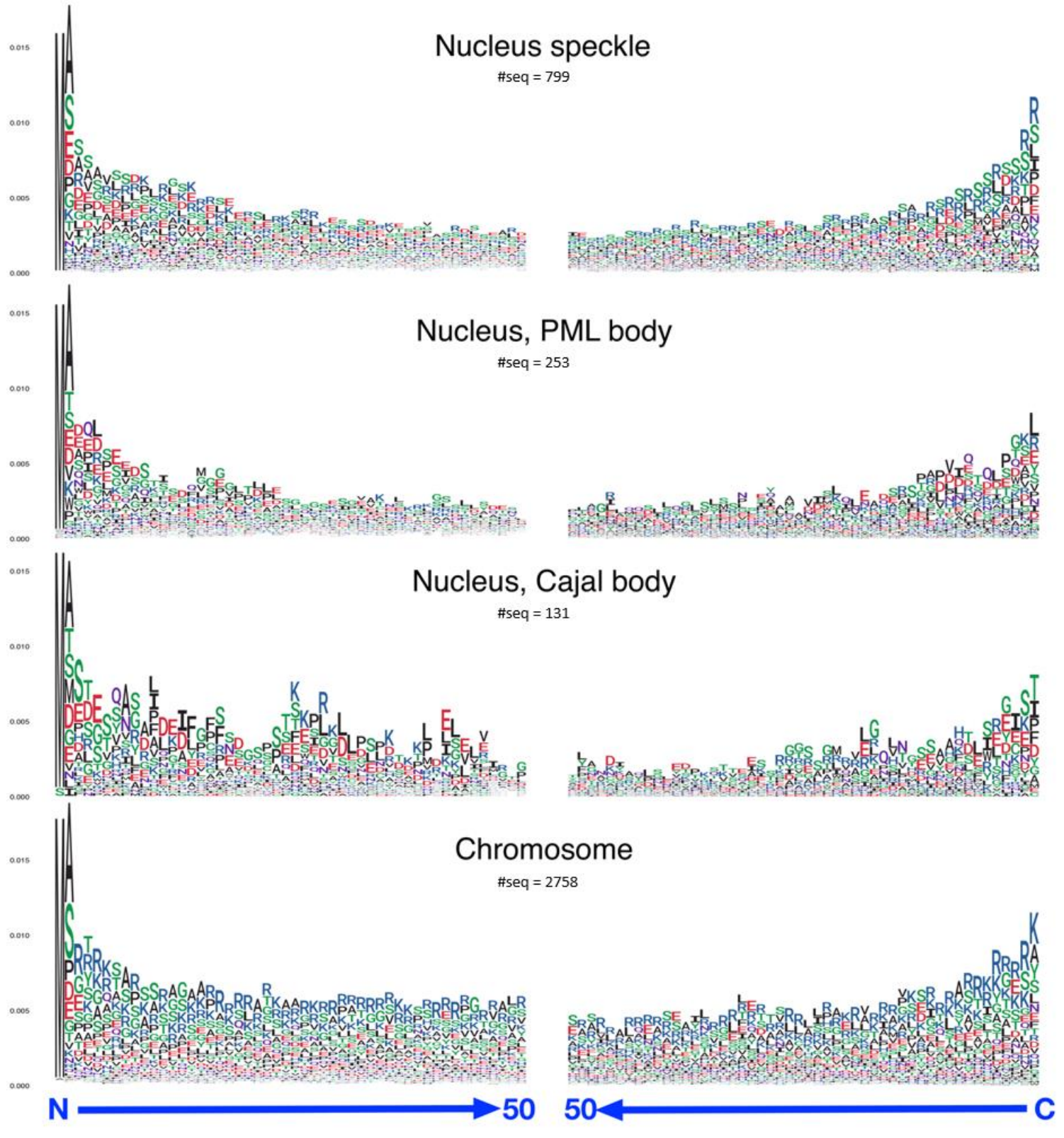

Figure A.2. The attention weights at the $\mathrm{N}$-terminus (left column) and the $\mathrm{C}$-terminus (right column) for protein localizations under the nucleus organelle (continue). 


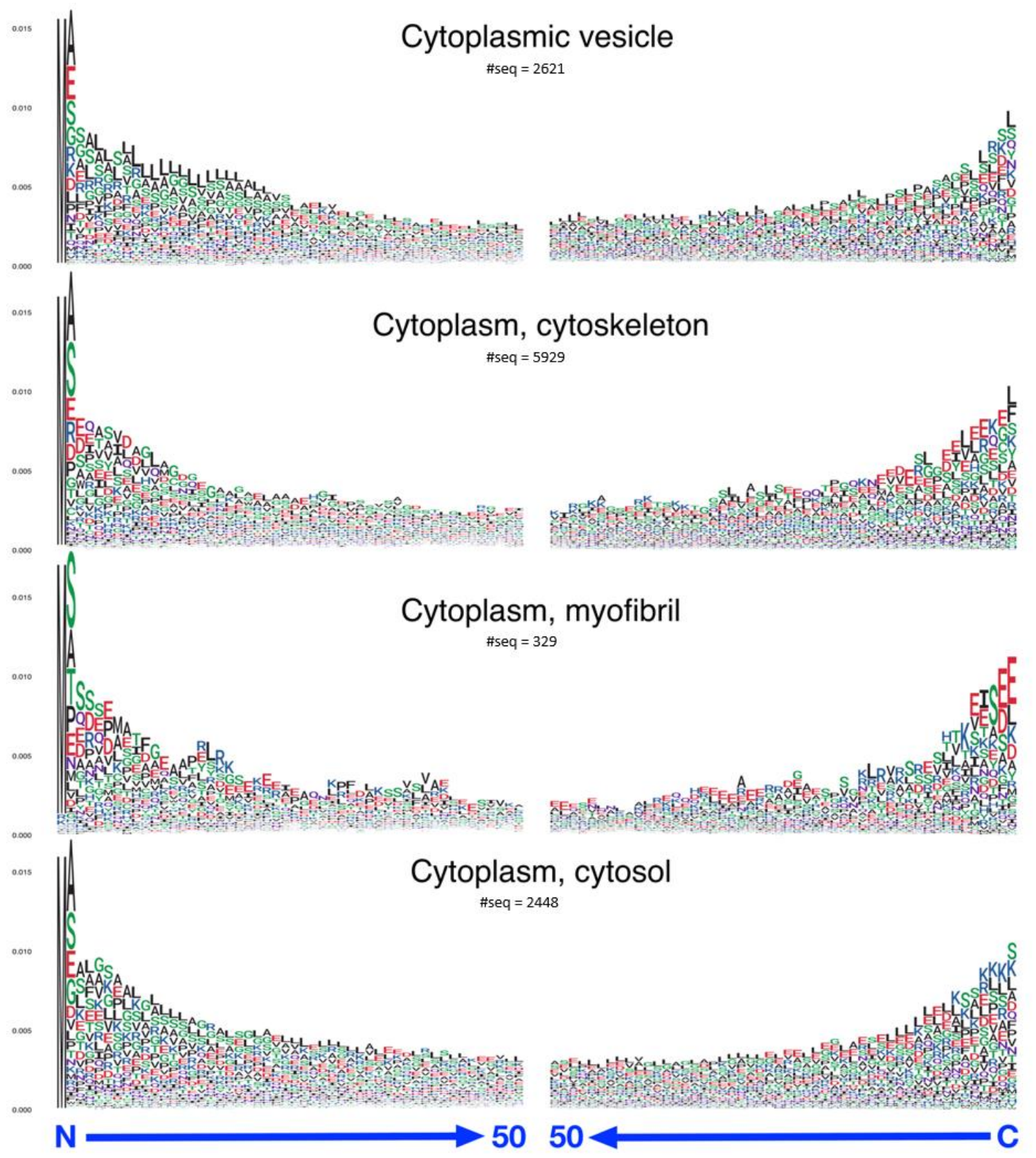

Figure A.3. The attention weights at the N-terminus (left column) and the C-terminus (right column) for protein localizations under the cytoplasm category. 


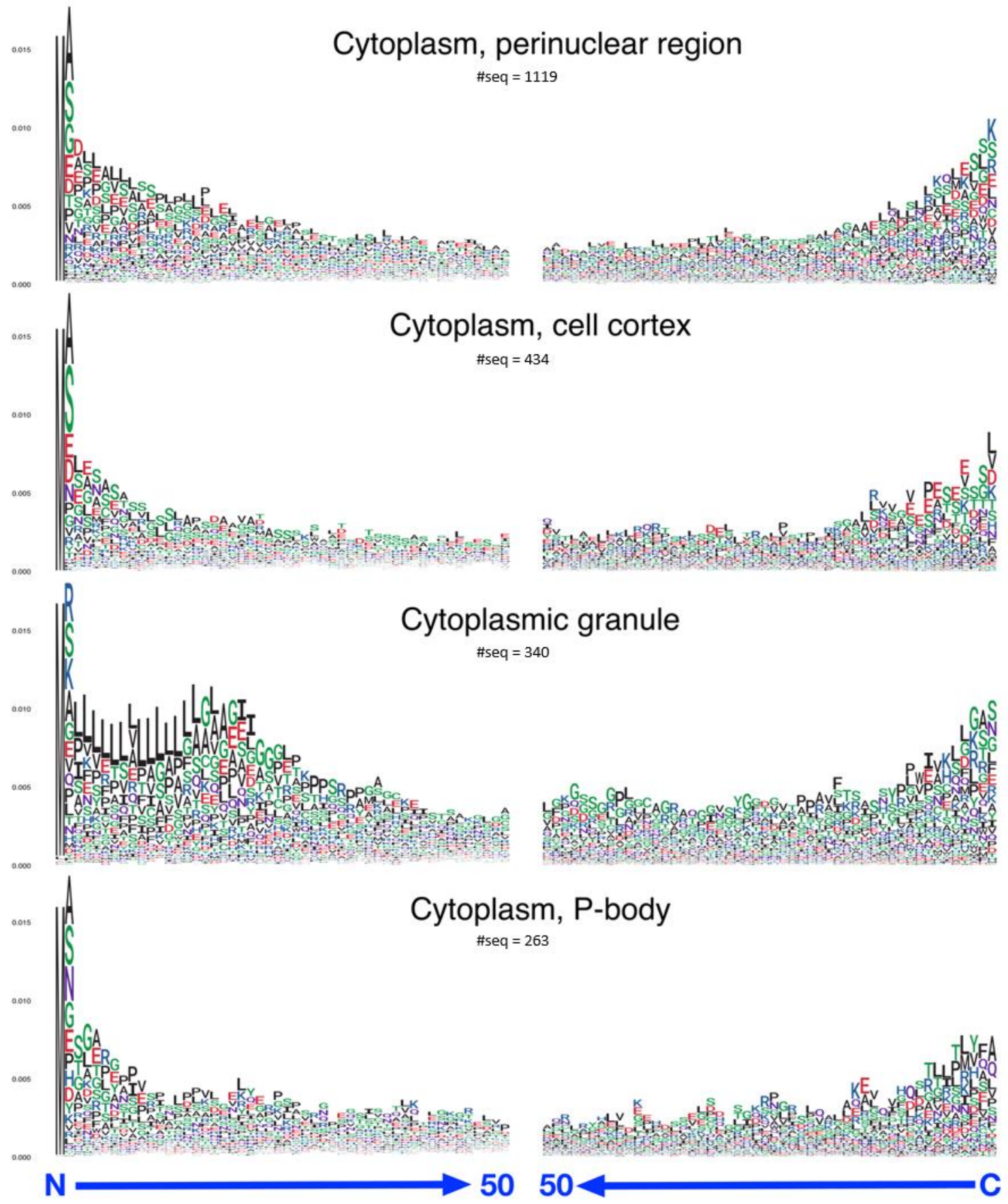

Figure A.4. The attention weights at the N-terminus (left column) and the C-terminus (right column) for protein localizations under the cytoplasm category. 


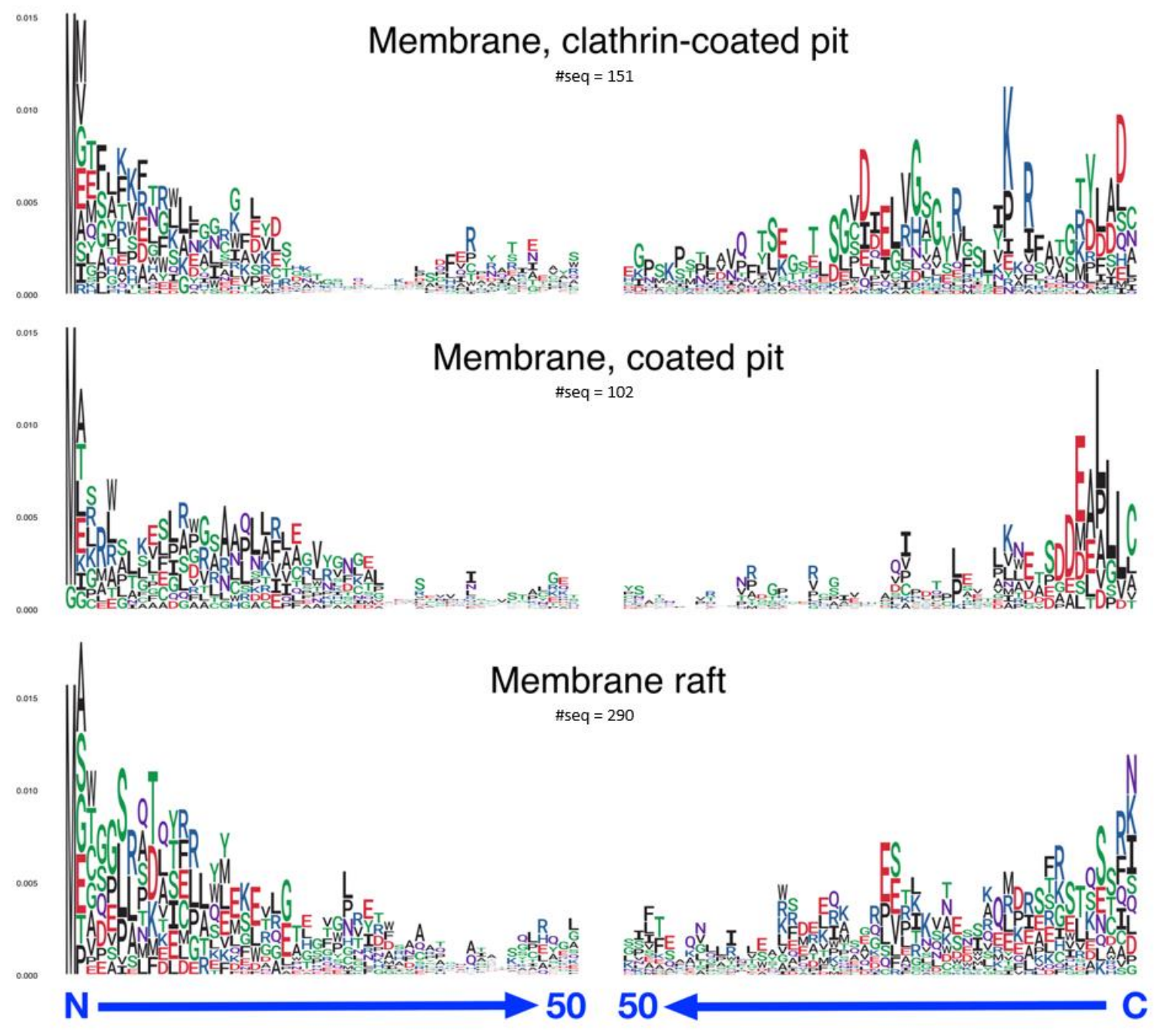

Figure A.5. The attention weights at the N-terminus (left column) and the C-terminus (right column) for protein localizations under the membrane category. 


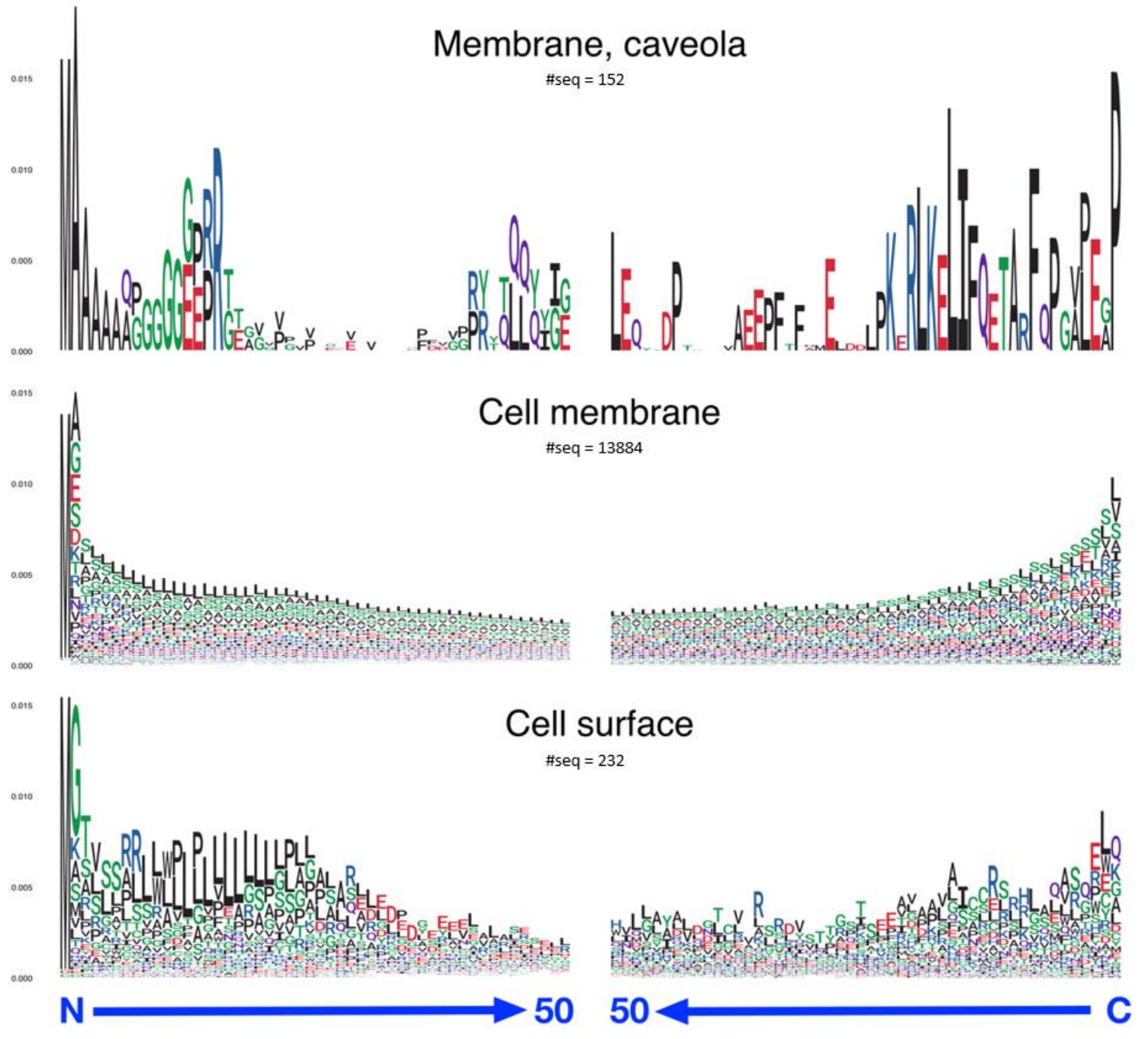

Figure A.6. The attention weights at the N-terminus (left column) and the C-terminus (right column) for protein localizations under the membrane category. 


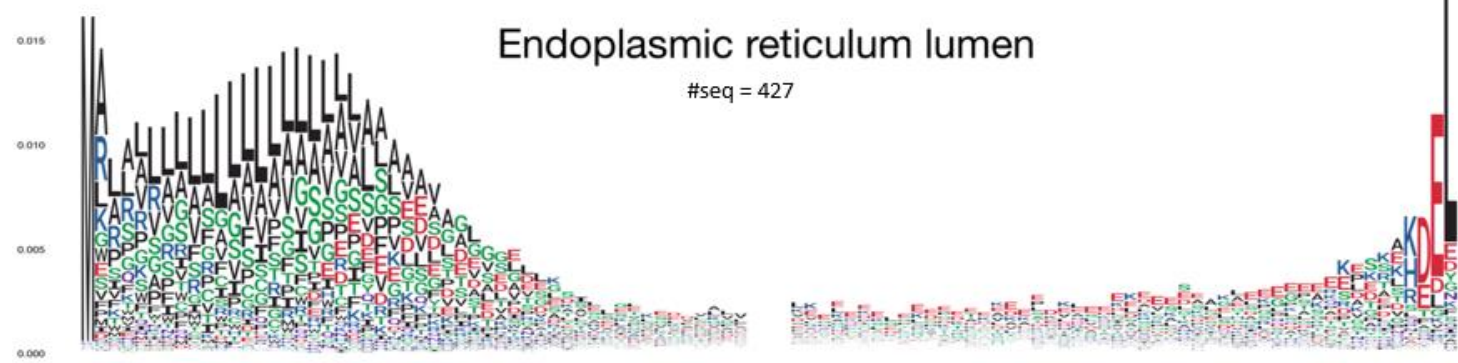

$\infty_{\infty}^{\infty}$

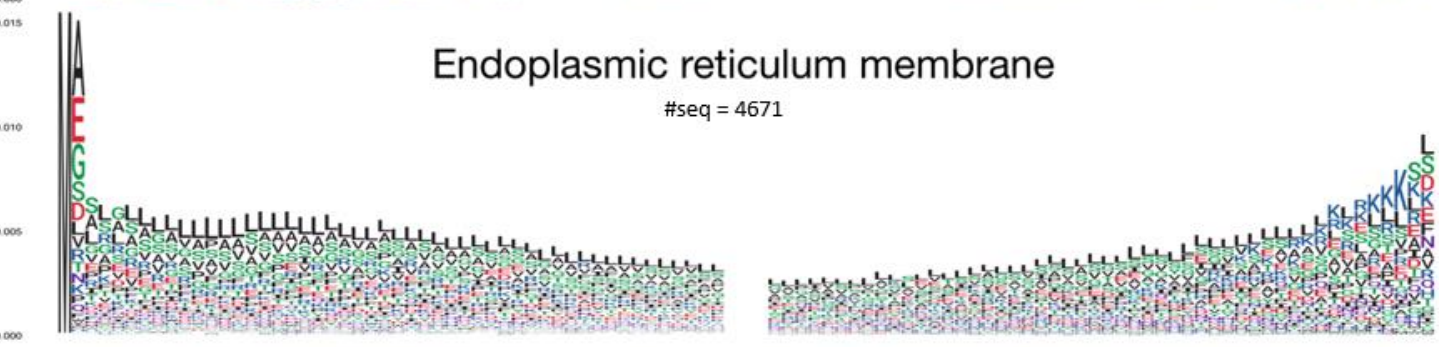

Golgi intermediate compartment

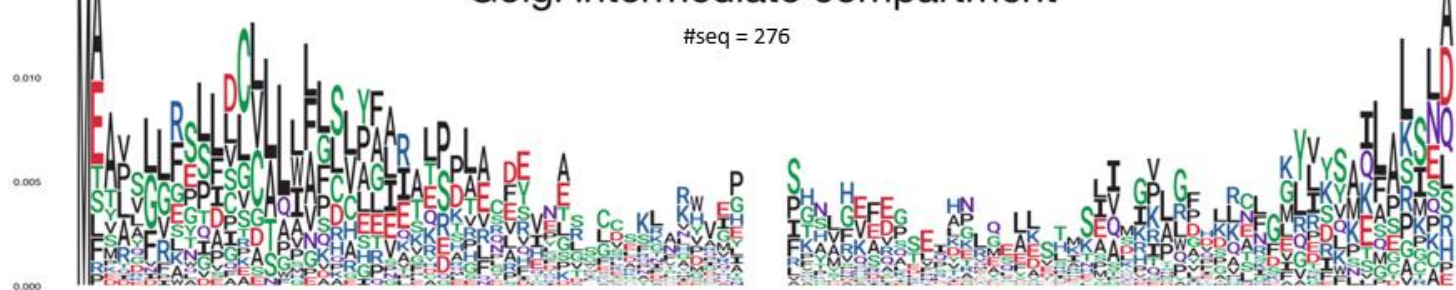

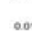
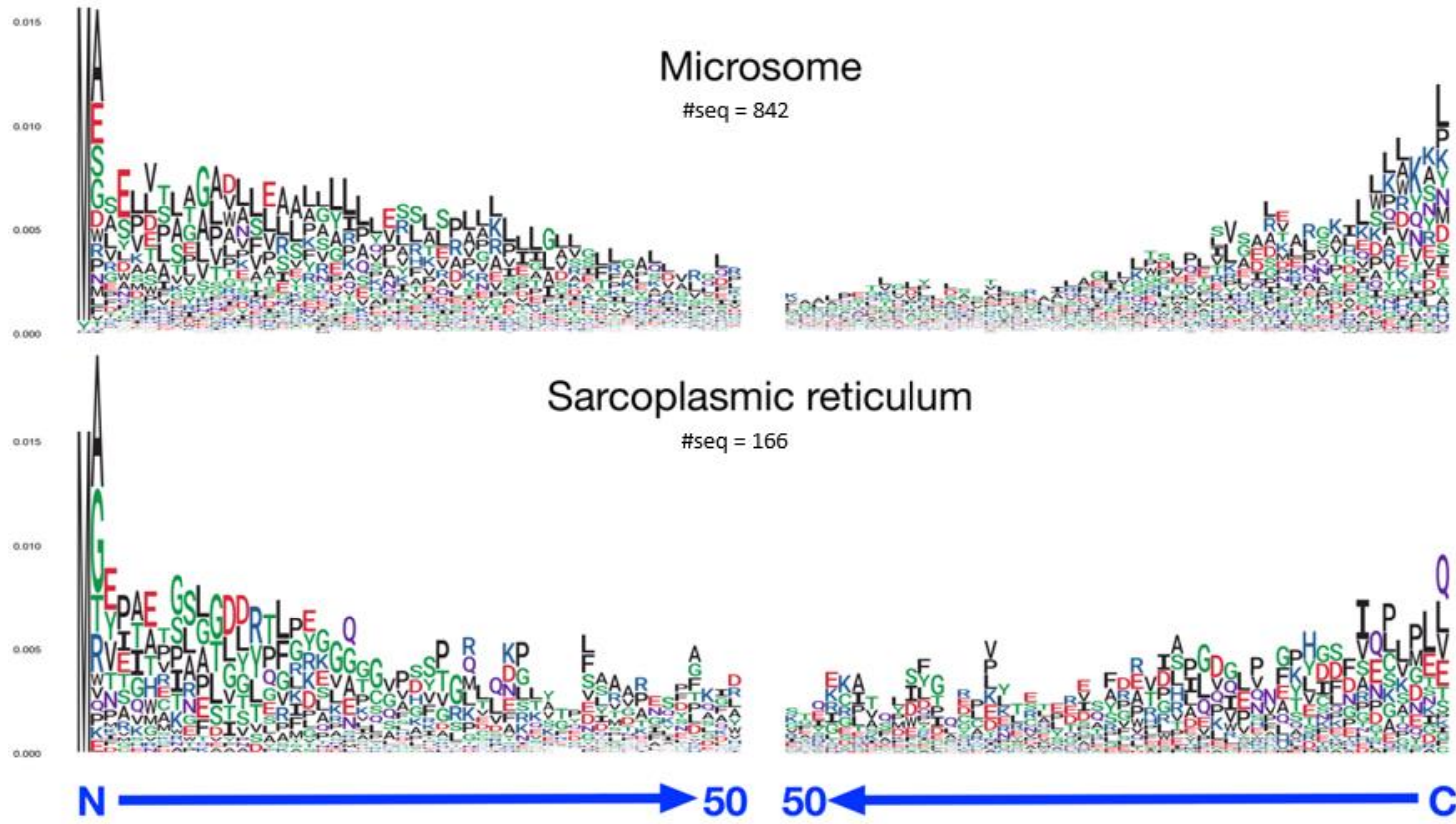

Figure A.7. The attention weights at the $\mathrm{N}$-terminus (left column) and the C-terminus (right column) for protein localizations under the endoplasmic reticulum organelle. 


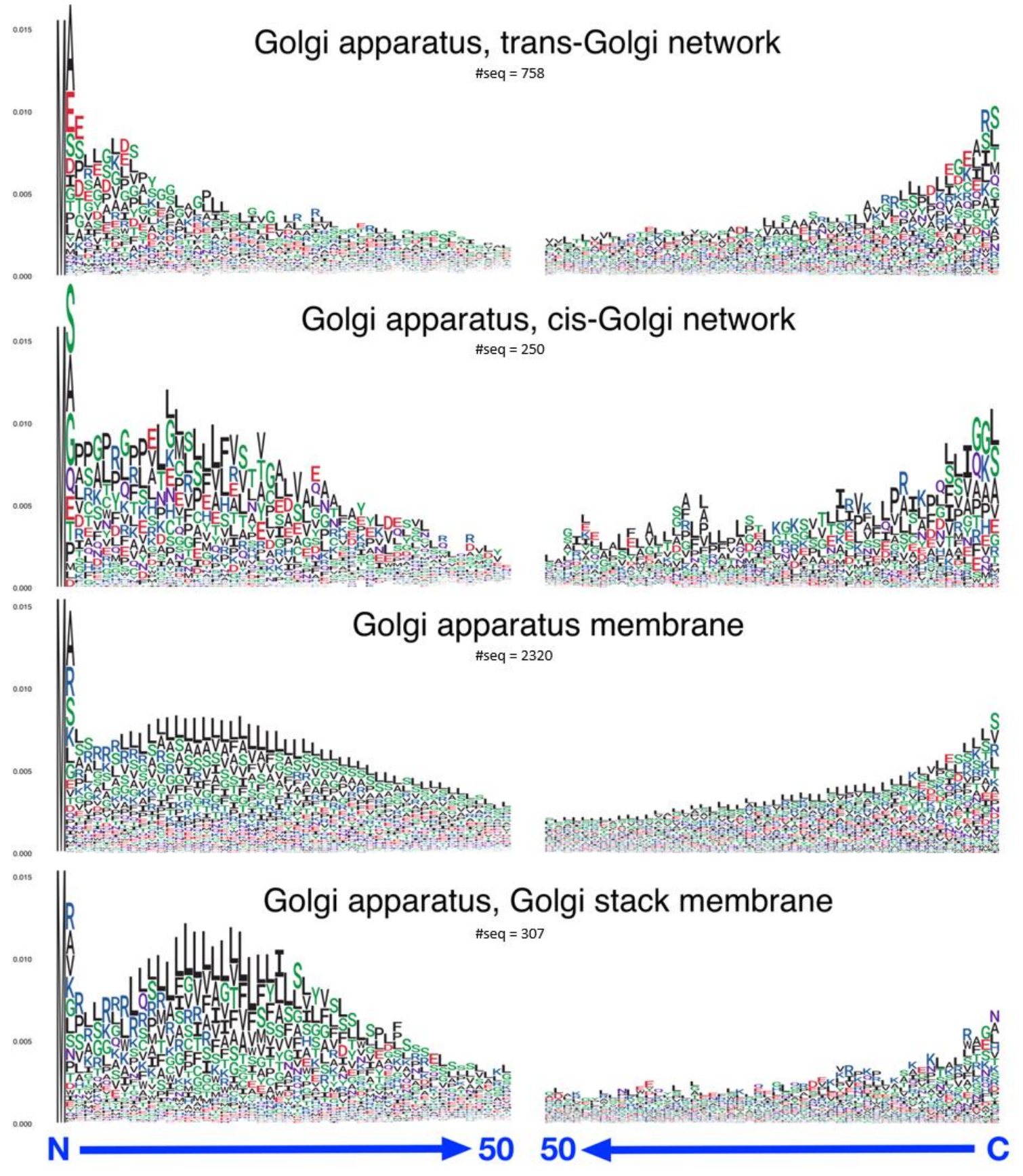

Figure A.8. The attention weights at the N-terminus (left column) and the C-terminus (right column) for protein localizations under the Golgi apparatus organelle. 


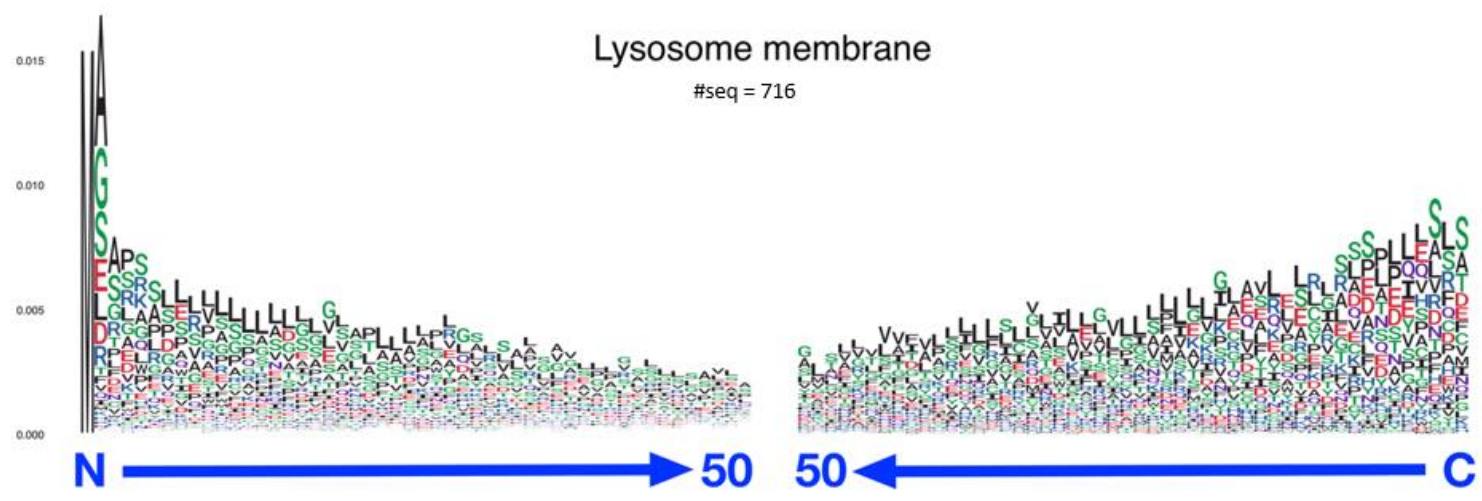

Figure A.9. The attention weights at the N-terminus (left column) and the C-terminus (right column) for protein localizations under the lysosome/vacuole organelle. 


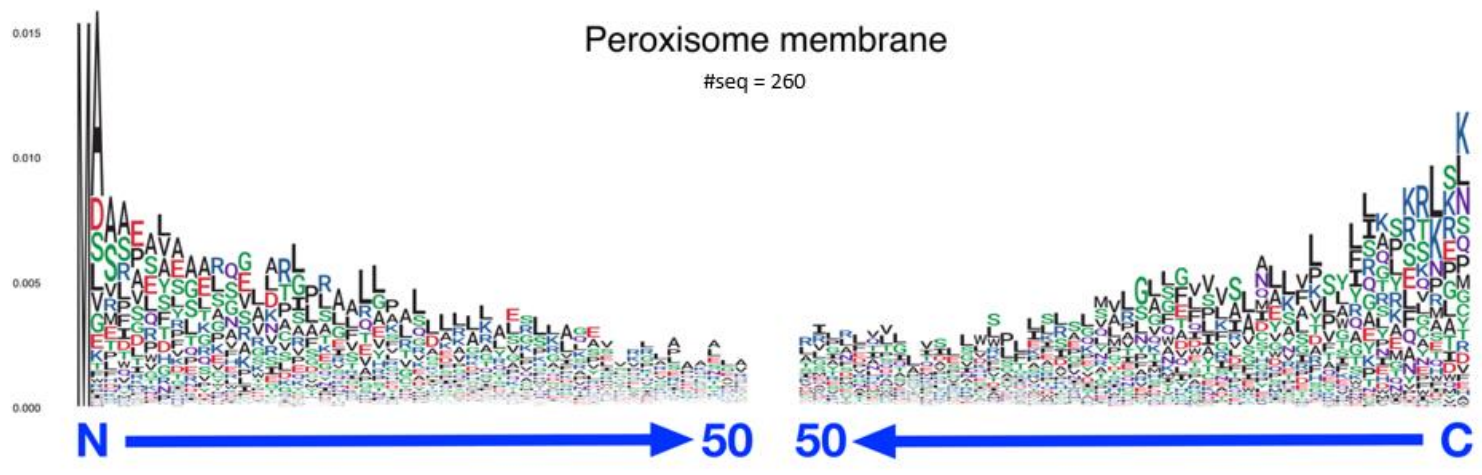

Figure A.10. The attention weights at the $\mathrm{N}$-terminus (left column) and the C-terminus (right column) for protein localizations under the peroxisome organelle. 


\section{APPENDIX B. GLAM2 ANALYSIS ON HIGH ATTENTION \\ WEIGHT FRAGMENTS FROM SUBCELLULAR \\ LOCALIZATION}

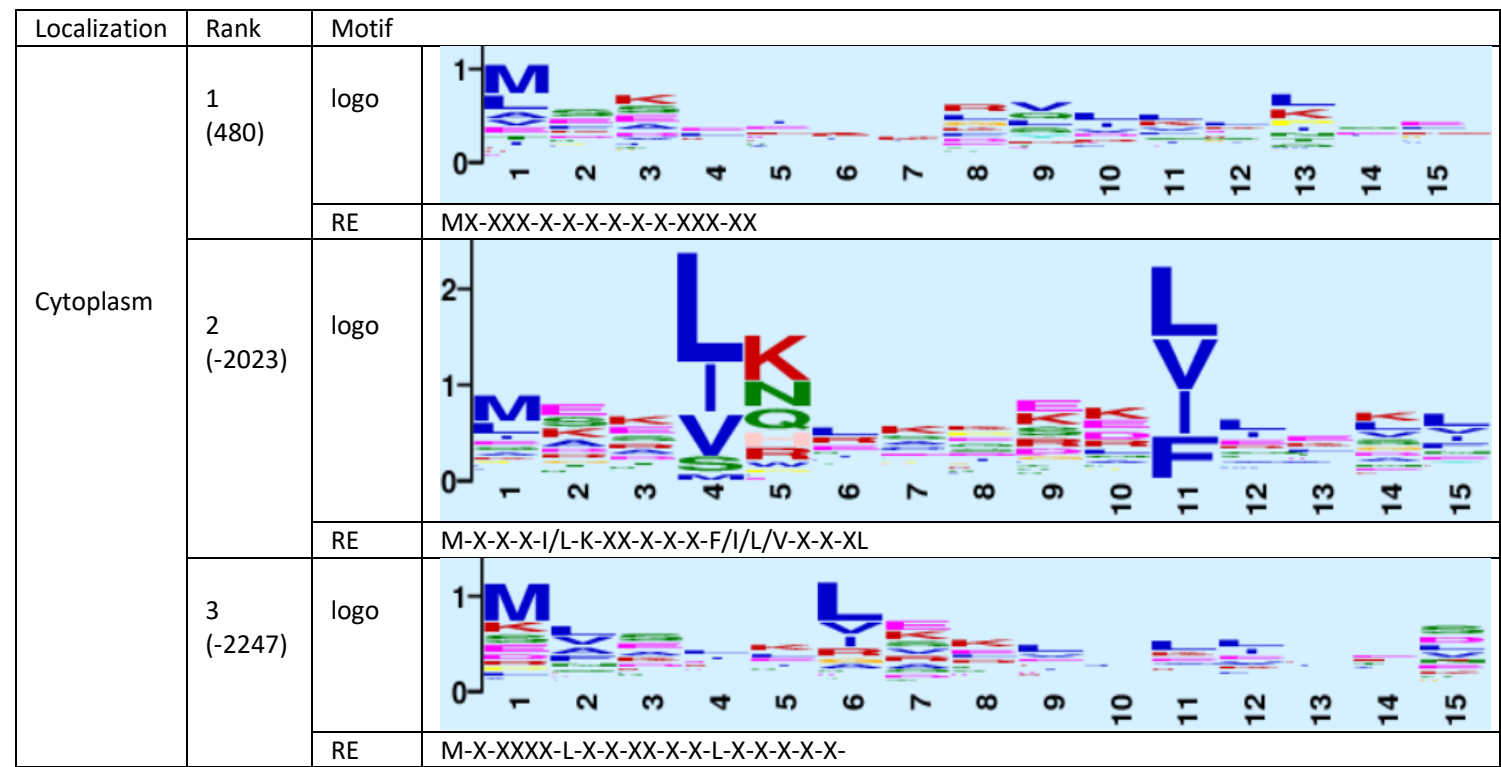

Figure B.1. The top 3 GLAM2 results for proteins in the cytoplasm. For each result, its rank, score, sequence logo, and the regular expression (RE) of the motif are given. 


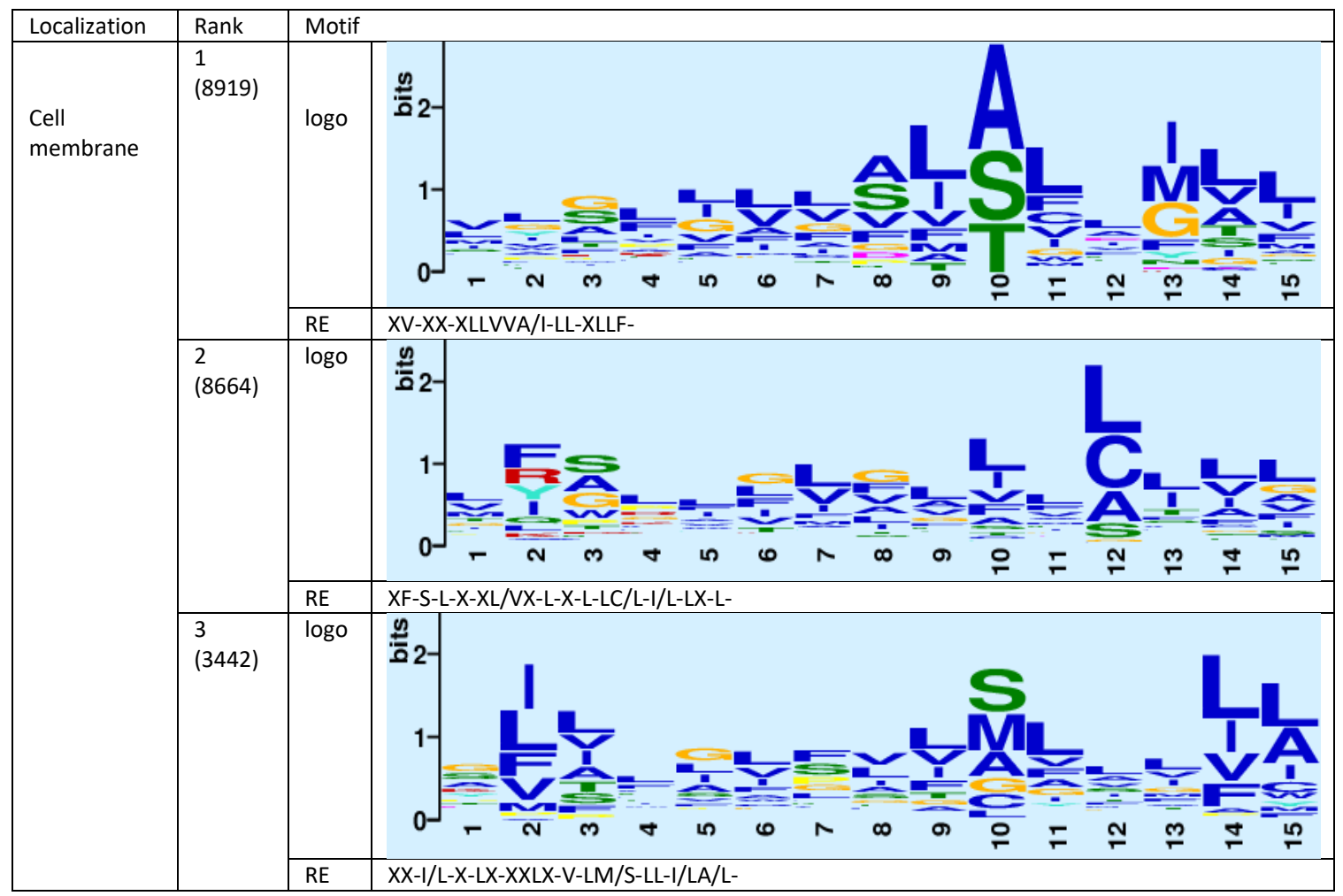

Figure B.2. The top 3 GLAM2 results for proteins in the cell membrane. For each result, its rank, score, sequence logo, and the regular expression (RE) of the motif are given. 


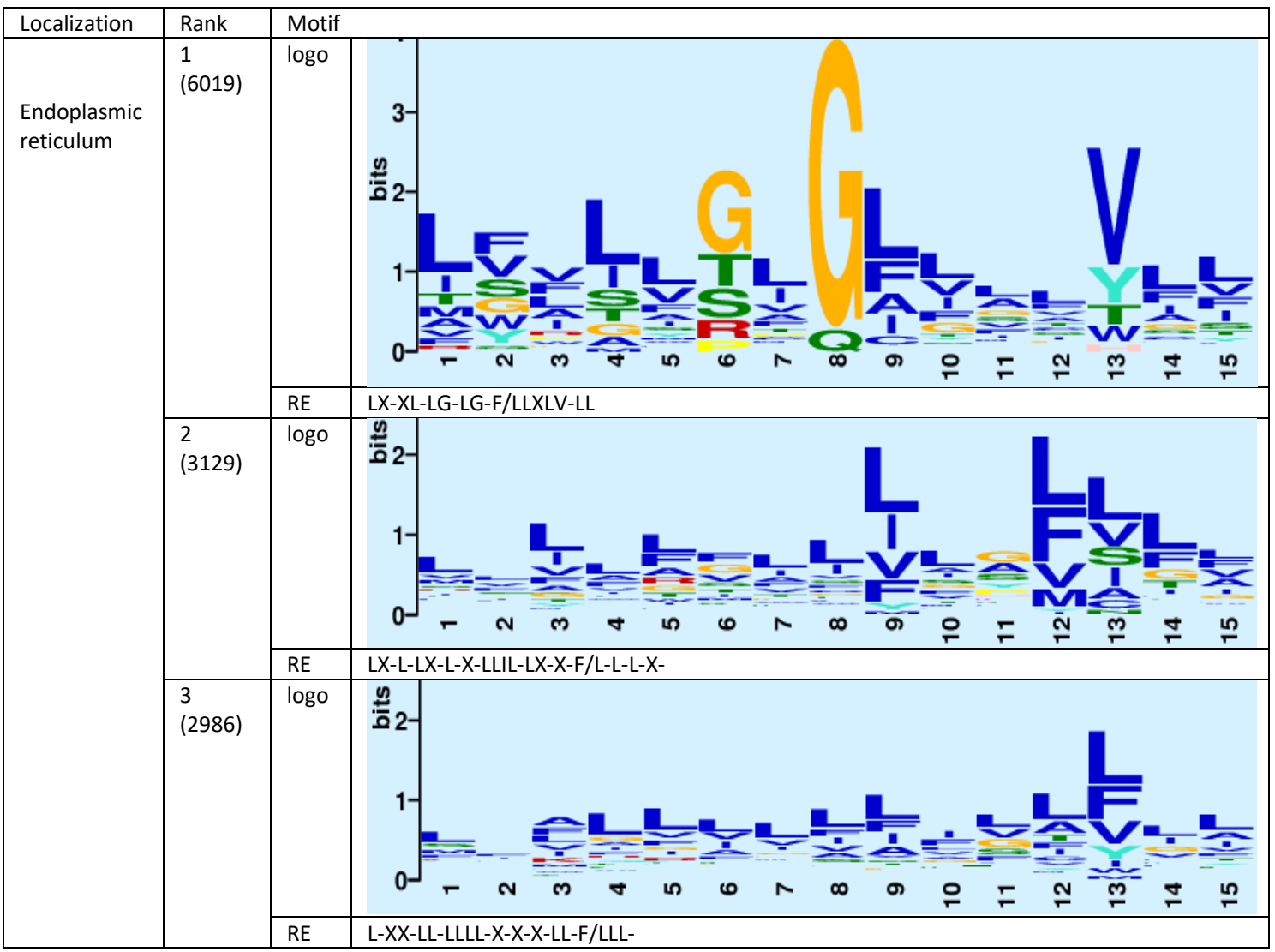

Figure B.3. The top 3 GLAM2 results for proteins in the endoplasmic reticulum. For each result, its rank, score, sequence logo, and the regular expression (RE) of the motif are given. 


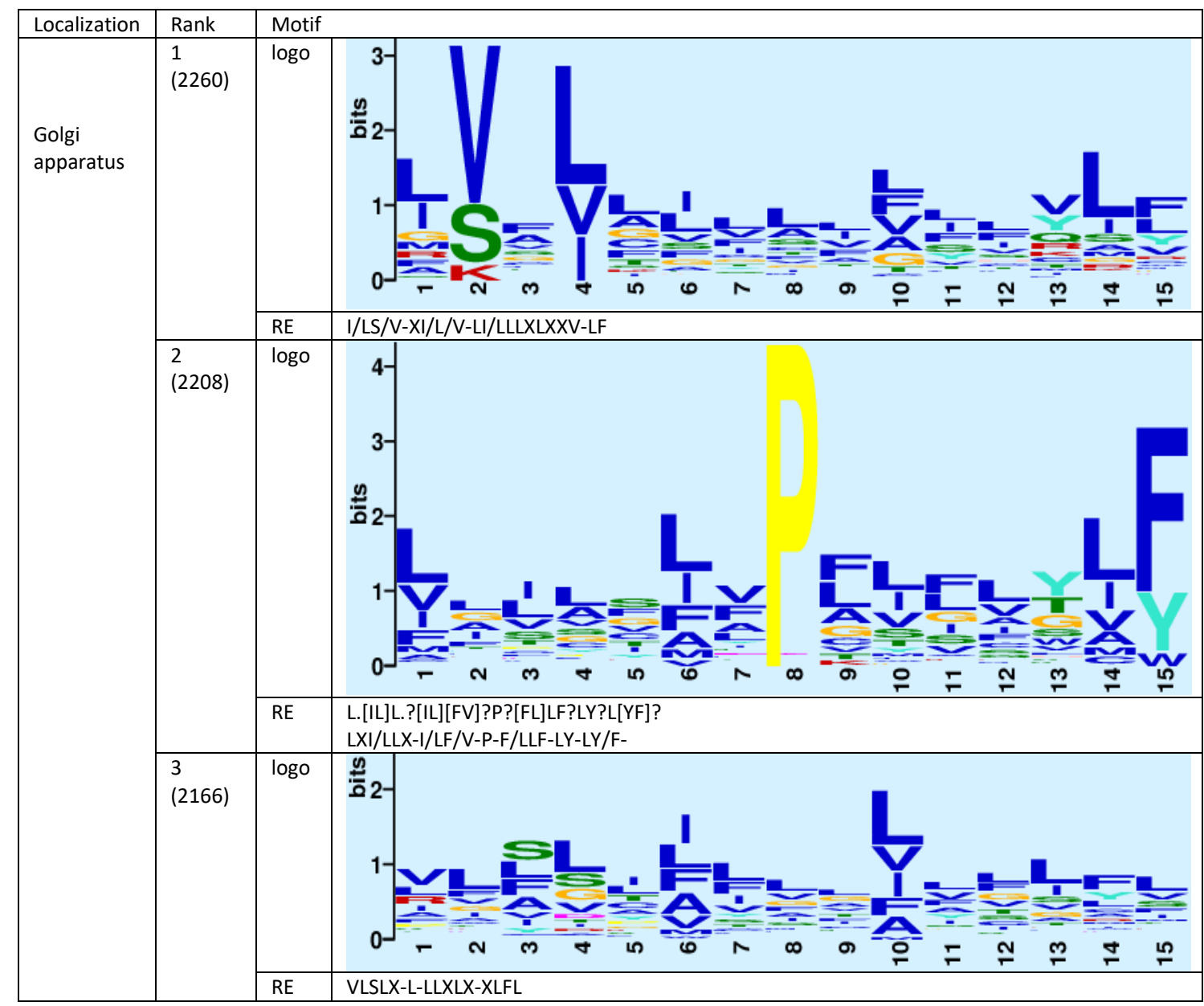

Figure B.4. The top 3 GLAM2 results for proteins in the Golgi apparatus. For each result, its rank, score, sequence logo, and the regular expression (RE) of the motif are given. 


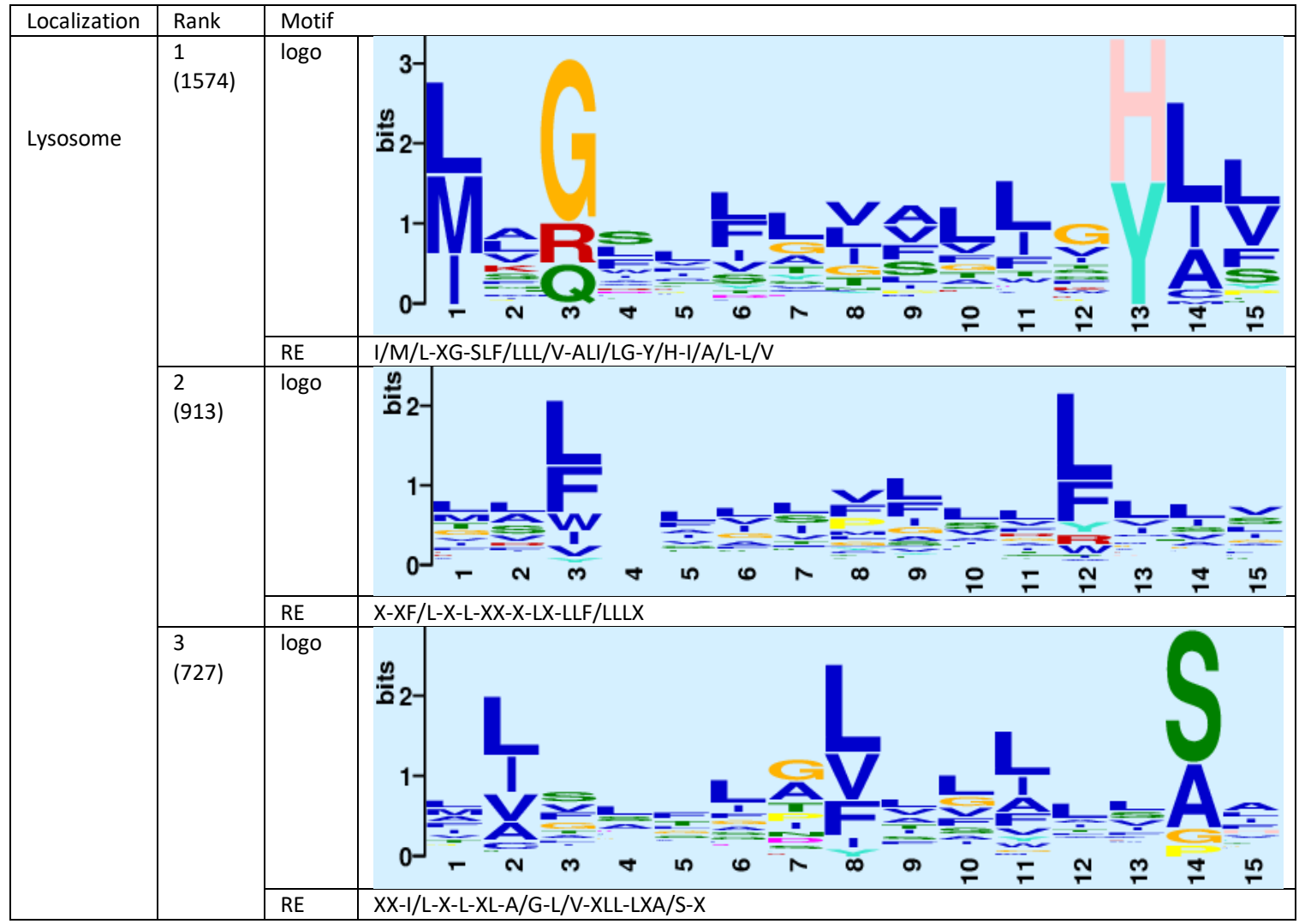

Figure B.5. The top 3 GLAM2 results for proteins in the lysosome. For each result, its rank, score, sequence logo, and the regular expression (RE) of the motif are given. 


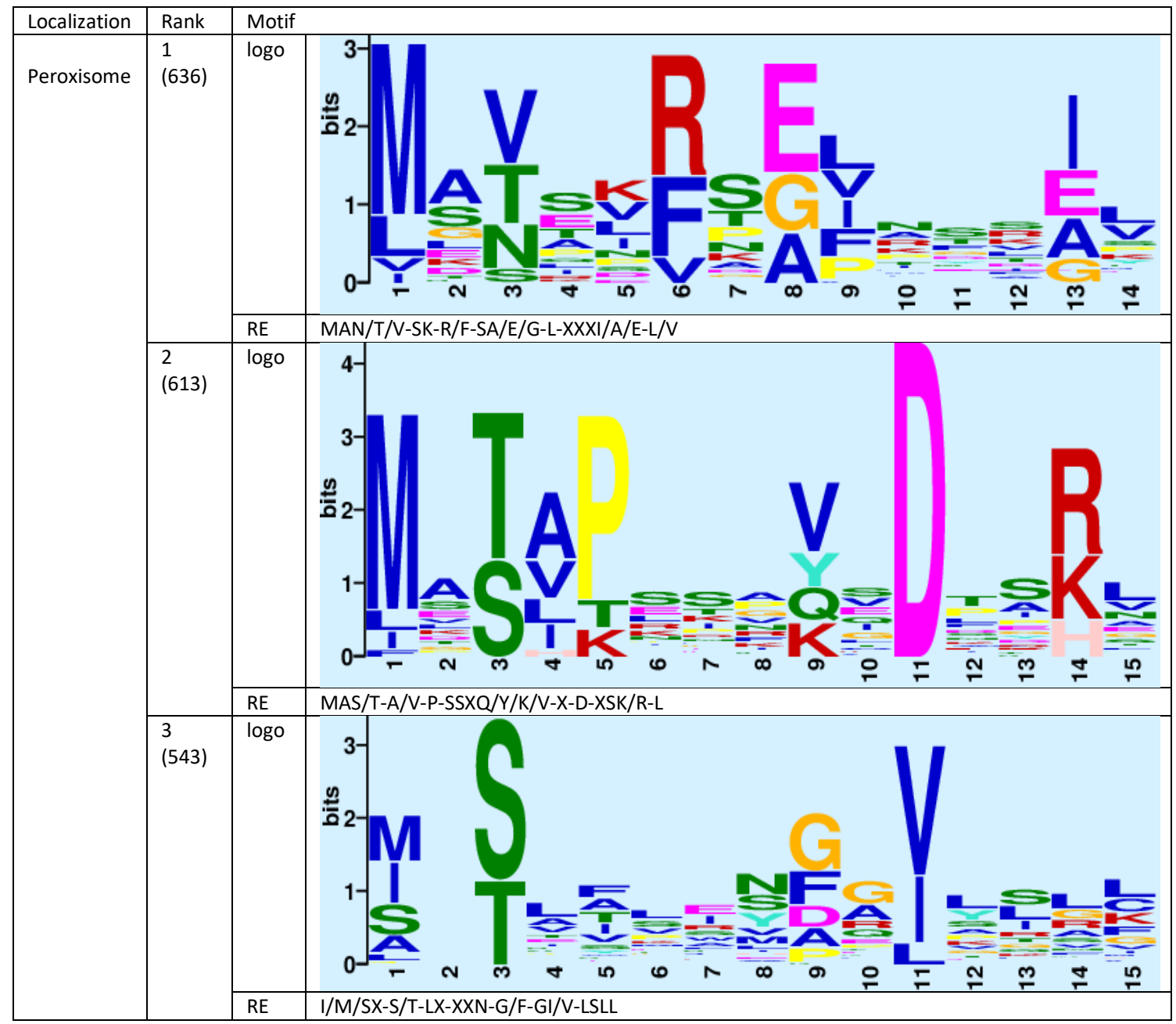

Figure B.6. The top 3 GLAM2 results for proteins in the peroxisome. For each result, its rank, score, sequence logo, and the regular expression (RE) of the motif are given. 


\section{BIBLIOGRAPHY}

[1] D. J. Schnell and D. N. Hebert, "Protein translocons: multifunctional mediators of protein translocation across membranes," Cell, vol. 112, no. 4, pp. 491-505, Feb 212003.

[2] W. Wickner and R. Schekman, "Protein translocation across biological membranes," Science, vol. 310, no. 5753, pp. 1452-6, Dec 22005.

[3] W. Neupert and J. M. Herrmann, "Translocation of proteins into mitochondria," Annu. Rev. Biochem, vol. 76, pp. 723-749, 2007.

[4] J. R. Davis, M. Kakar, and C. S. Lim, "Controlling protein compartmentalization to overcome disease," Pharm Res, vol. 24, no. 1, pp. 17-27, Jan 2007.

[5] M. C. Hung and W. Link, "Protein localization in disease and therapy," J Cell Sci, vol. 124, no. Pt 20, pp. 3381-92, Oct 152011.

[6] J. A. Rodriguez, S. Schüchner, W. W. Au, M. Fabbro, and B. R. Henderson, "Nuclear-cytoplasmic shuttling of BARD1 contributes to its proapoptotic activity and is regulated by dimerization with BRCA1," Oncogene, vol. 23, no. 10, pp. 1809-1820, 2004.

[7] M. M. Marques-Bueno, J. Moreno-Romero, L. Abas, R. De Michele, and M. C. Martinez, "A dominant negative mutant of protein kinase CK2 exhibits altered auxin responses in Arabidopsis," Plant J, vol. 67, no. 1, pp. 169-80, Jul 2011.

[8] K. Thevissen et al., "The plant defensin RsAFP2 induces cell wall stress, septin mislocalization and accumulation of ceramides in Candida albicans," Mol Microbiol, vol. 84, no. 1, pp. 166-80, Apr 2012.

[9] T. A. Rapoport, "Protein translocation across the eukaryotic endoplasmic reticulum and bacterial plasma membranes," Nature, vol. 450, no. 7170, pp. 6639, Nov 292007.

[10] O. Schmidt, N. Pfanner, and C. Meisinger, "Mitochondrial protein import: from proteomics to functional mechanisms," Nat Rev Mol Cell Biol, vol. 11, no. 9, pp. 655-67, Sep 2010.

[11] M. Suntharalingam and S. R. Wente, "Peering through the pore: nuclear pore complex structure, assembly, and function," Developmental cell, vol. 4, no. 6, pp. 775-789, 2003. 
[12] L. J. Terry, E. B. Shows, and S. R. Wente, "Crossing the nuclear envelope: hierarchical regulation of nucleocytoplasmic transport," Science, vol. 318, no. 5855, pp. 1412-6, Nov 302007.

[13] J. Wolf, W. Schliebs, and R. Erdmann, "Peroxisomes as dynamic organelles: peroxisomal matrix protein import," The FEBS journal, vol. 277, no. 16, pp. 3268-3278, 2010.

[14] N. C. Bauer, P. W. Doetsch, and A. H. Corbett, "Mechanisms Regulating Protein Localization," Traffic, vol. 16, no. 10, pp. 1039-61, Oct 2015.

[15] M. Hagmann, "Protein zip codes make Nobel journey," Science, vol. 286, 1999.

[16] A. Chacinska, C. M. Koehler, D. Milenkovic, T. Lithgow, and N. Pfanner, "Importing mitochondrial proteins: machineries and mechanisms," Cell, vol. 138, no. 4, pp. 628-44, Aug 212009.

[17] G. Blobel and B. Dobberstein, "Transfer of proteins across membranes. I. Presence of proteolytically processed and unprocessed nascent immunoglobulin light chains on membrane-bound ribosomes of murine myeloma," J Cell Biol, vol. 67, no. 3, pp. 835-51, Dec 1975.

[18] L. Jakobsen et al., "Novel asymmetrically localizing components of human centrosomes identified by complementary proteomics methods," EMBO J, vol. 30, no. 8, pp. 1520-35, Apr 202011.

[19] A. Christoforou et al., "A draft map of the mouse pluripotent stem cell spatial proteome," Nat Commun, vol. 7, p. 8992, Jan 122016.

[20] D. N. Itzhak, S. Tyanova, J. Cox, and G. H. Borner, "Global, quantitative and dynamic mapping of protein subcellular localization," Elife, vol. 5, Jun 92016.

[21] L. M. Orre et al., "SubCellBarCode: Proteome-wide Mapping of Protein Localization and Relocalization," Mol Cell, vol. 73, no. 1, pp. 166-182 e7, Jan 3 2019.

[22] H. W. Rhee et al., "Proteomic mapping of mitochondria in living cells via spatially restricted enzymatic tagging," Science, vol. 339, no. 6125, pp. 13281331, Mar 152013.

[23] V. Hung et al., "Proteomic mapping of the human mitochondrial intermembrane space in live cells via ratiometric APEX tagging," Mol Cell, vol. 55, no. 2, pp. 332-41, Jul 172014.

[24] S. Y. Lee, M. G. Kang, J. S. Park, G. Lee, A. Y. Ting, and H. W. Rhee, "APEX Fingerprinting Reveals the Subcellular Localization of Proteins of Interest," Cell Rep, vol. 15, no. 8, pp. 1837-47, May 242016. 
[25] J. D. Martell et al., "Engineered ascorbate peroxidase as a genetically encoded reporter for electron microscopy," vol. 30, no. 11, pp. 1143-1148, 2012.

[26] Y. T. Chong et al., "Yeast Proteome Dynamics from Single Cell Imaging and Automated Analysis," Cell, vol. 161, no. 6, pp. 1413-24, Jun 42015.

[27] L. Barbe et al., "Toward a confocal subcellular atlas of the human proteome," Mol Cell Proteomics, vol. 7, no. 3, pp. 499-508, Mar 2008.

[28] C. Stadler, M. Skogs, H. Brismar, M. Uhlen, and E. Lundberg, "A single fixation protocol for proteome-wide immunofluorescence localization studies," $J$ Proteomics, vol. 73, no. 6, pp. 1067-78, Apr 182010.

[29] P. J. Thul et al., "A subcellular map of the human proteome," Science, vol. 356, no. 6340, May 262017.

[30] T. J. Burns et al., "High-throughput precision measurement of subcellular localization in single cells," Cytometry A, vol. 91, no. 2, pp. 180-189, Feb 2017.

[31] S. Hochreiter and J. Schmidhuber, "Long short-term memory," Neural computation, vol. 9, no. 8, pp. 1735-1780, 1997.

[32] Z. Lin et al., "A structured self-attentive sentence embedding," arXiv preprint, 2017.

[33] C. UniProt, "UniProt: a worldwide hub of protein knowledge," Nucleic Acids Res, vol. 47, no. D1, pp. D506-D515, Jan 82019.

[34] J. L. Gardy and F. S. Brinkman, "Methods for predicting bacterial protein subcellular localization," Nat Rev Microbiol, vol. 4, no. 10, pp. 741-51, Oct 2006.

[35] K. Nakai, "Protein sorting signals and prediction of subcellular localization," Adv Protein Chem, vol. 54, pp. 277-344, 2000.

[36] K. Imai and K. Nakai, "Tools for the Recognition of Sorting Signals and the Prediction of Subcellular Localization of Proteins From Their Amino Acid Sequences," Front Genet, vol. 11, p. 607812, 2020.

[37] Y. Shen, Y. Ding, J. Tang, Q. Zou, and F. Guo, "Critical evaluation of web-based prediction tools for human protein subcellular localization," Brief Bioinform, vol. 21, no. 5, pp. 1628-1640, Sep 252020.

[38] P. Donnes and A. Hoglund, "Predicting protein subcellular localization: past, present, and future," Genomics Proteomics Bioinformatics, vol. 2, no. 4, pp. 20915, Nov 2004.

[39] K. C. Chou and H. B. Shen, "Recent progress in protein subcellular location prediction," Anal Biochem, vol. 370, no. 1, pp. 1-16, Nov 12007. 
[40] Z. Wang, Q. Zou, Y. Jiang, Y. Ju, and X. Zeng, "Review of protein subcellular localization prediction," J Current Bioinformatics, vol. 9, no. 3, pp. 331-342, 2014.

[41] K. C. Chou, "A novel approach to predicting protein structural classes in a (20-1)D amino acid composition space," Proteins, vol. 21, no. 4, pp. 319-44, Apr 1995.

[42] K. Chou, "Prediction of protein cellular attributes using pseudo-amino acid composition," Proteins: Structure, Function, Bioinformatics, vol. 43, no. 3, pp. 246-255, 2001.

[43] R. Nair and B. Rost, "Sequence conserved for subcellular localization," Protein Sci, vol. 11, no. 12, pp. 2836-47, Dec 2002.

[44] T. Joshi and D. Xu, "Quantitative assessment of relationship between sequence similarity and function similarity," J BMC genomics, vol. 8, no. 1, pp. 1-10, 2007.

[45] S. F. Altschul, W. Gish, W. Miller, E. W. Myers, and D. J. Lipman, "Basic local alignment search tool," Mol Biol, vol. 215, no. 3, pp. 403-10, Oct 51990.

[46] M. Remmert, A. Biegert, A. Hauser, and J. Soding, "HHblits: lightning-fast iterative protein sequence searching by HMM-HMM alignment," Nat Methods, vol. 9, no. 2, pp. 173-5, Dec 252011.

[47] M. Ashburner et al., "Gene ontology: tool for the unification of biology. The Gene Ontology Consortium," Nat Genet, vol. 25, no. 1, pp. 25-9, May 2000.

[48] T. Goldberg et al., "LocTree3 prediction of localization," Nucleic Acids Res, vol. 42, no. Web Server issue, pp. W350-5, Jul 2014.

[49] S. Briesemeister, T. Blum, S. Brady, Y. Lam, O. Kohlbacher, and H. Shatkay, "SherLoc2: a high-accuracy hybrid method for predicting subcellular localization of proteins," Journal of proteome research, vol. 8, no. 11, pp. 5363-5366, 2009.

[50] T. Blum, S. Briesemeister, and O. Kohlbacher, "MultiLoc2: integrating phylogeny and Gene Ontology terms improves subcellular protein localization prediction," BMC bioinformatics, vol. 10, no. 1, p. 274, 2009.

[51] S. Briesemeister, J. Rahnenfuhrer, and O. Kohlbacher, "YLoc--an interpretable web server for predicting subcellular localization," Nucleic Acids Res, vol. 38, no. Web Server issue, pp. W497-502, Jul 2010.

[52] J. L. Gardy et al., "PSORT-B: Improving protein subcellular localization prediction for Gram-negative bacteria," Nucleic Acids Res, vol. 31, no. 13, pp. 3613-7, Jul 12003. 
[53] C. J. Sigrist et al., "PROSITE: a documented database using patterns and profiles as motif descriptors," J Briefings in bioinformatics, vol. 3, no. 3, pp. 265-274, 2002.

[54] J. J. Almagro Armenteros et al., "SignalP 5.0 improves signal peptide predictions using deep neural networks," Nat Biotechnol, vol. 37, no. 4, pp. 420-423, Apr 2019.

[55] J. J. Almagro Armenteros et al., "Detecting sequence signals in targeting peptides using deep learning," Life Sci Alliance, vol. 2, no. 5, Oct 2019.

[56] H. Lin, W. Chen, L. Yuan, Z. Li, and H. Ding, "Using over-represented tetrapeptides to predict protein submitochondria locations," Acta biotheoretica, vol. 61, no. 2, pp. 259-268, 2013.

[57] H. Ding et al., "Prediction of Golgi-resident protein types by using feature selection technique," J Chemometrics Intelligent Laboratory Systems, vol. 124, pp. 9-13, 2013.

[58] J. Sperschneider et al., "LOCALIZER: subcellular localization prediction of both plant and effector proteins in the plant cell," Scientific reports, vol. 7, no. 1, pp. 114, 2017.

[59] M. S. Venkatarajan and W. Braun, "New quantitative descriptors of amino acids based on multidimensional scaling of a large number of physical-chemical properties," Molecular modeling annual, vol. 7, no. 12, pp. 445-453, 2001.

[60] S. Kawashima, H. Ogata, and M. Kanehisa, "AAindex: amino acid index database," J Nucleic acids research, vol. 27, no. 1, pp. 368-369, 1999.

[61] L. Licata et al., "MINT, the molecular interaction database: 2012 update," Nucleic Acids Res, vol. 40, no. Database issue, pp. D857-61, Jan 2012.

[62] I. Xenarios, L. Salwinski, X. J. Duan, P. Higney, S. M. Kim, and D. Eisenberg, "DIP, the Database of Interacting Proteins: a research tool for studying cellular networks of protein interactions," Nucleic Acids Res, vol. 30, no. 1, pp. 303-5, Jan 12002.

[63] R. Oughtred et al., "The BioGRID interaction database: 2019 update," Nucleic Acids Res, vol. 47, no. D1, pp. D529-D541, Jan 82019.

[64] D. Szklarczyk et al., "STRING v11: protein-protein association networks with increased coverage, supporting functional discovery in genome-wide experimental datasets," Nucleic Acids Res, vol. 47, no. D1, pp. D607-D613, Jan 8 2019.

[65] M. M. Ananda and J. Hu, "NetLoc: Network based protein localization prediction using protein-protein interaction and co-expression networks," in 2010 IEEE 
International Conference on Bioinformatics and Biomedicine (BIBM), 2010, pp. 142-148: IEEE.

[66] N. Zhang et al., "MU-LOC: A Machine-Learning Method for Predicting Mitochondrially Localized Proteins in Plants," Front Plant Sci, vol. 9, p. 634, 2018.

[67] M. Ryngajllo et al., "SLocX: Predicting Subcellular Localization of Arabidopsis Proteins Leveraging Gene Expression Data," Front Plant Sci, vol. 2, p. 43, 2011.

[68] R. Edgar, M. Domrachev, and A. E. Lash, "Gene Expression Omnibus: NCBI gene expression and hybridization array data repository," Nucleic Acids Res, vol. 30, no. 1, pp. 207-10, Jan 12002.

[69] K. Tomczak, P. Czerwinska, and M. Wiznerowicz, "The Cancer Genome Atlas (TCGA): an immeasurable source of knowledge," Contemp Oncol (Pozn), vol. 19, no. 1A, pp. A68-77, 2015.

[70] C. Cortes and V. Vapnik, "Support-vector networks," J Machine learning, vol. 20, no. 3, pp. 273-297, 1995.

[71] S. Hua and Z. Sun, "Support vector machine approach for protein subcellular localization prediction," J Bioinformatics, vol. 17, no. 8, pp. 721-728, 2001.

[72] D. Sarda, G. H. Chua, K. B. Li, and A. Krishnan, "pSLIP: SVM based protein subcellular localization prediction using multiple physicochemical properties," BMC Bioinformatics, vol. 6, p. 152, Jun 172005.

[73] D. M. Tax and R. P. Duin, "Support vector data description," J Machine learning, vol. 54, no. 1, pp. 45-66, 2004.

[74] K. Lee, D. W. Kim, D. Na, K. H. Lee, and D. Lee, "PLPD: reliable protein localization prediction from imbalanced and overlapped datasets," Nucleic Acids Res, vol. 34, no. 17, pp. 4655-66, 2006.

[75] C. S. Yu, Y. C. Chen, C. H. Lu, and J. K. Hwang, "Prediction of protein subcellular localization," Proteins, vol. 64, no. 3, pp. 643-51, Aug 152006.

[76] C. S. Yu et al., "CELLO2GO: a web server for protein subCELlular LOcalization prediction with functional gene ontology annotation," PLoS One, vol. 9, no. 6, p. e99368, 2014.

[77] X. Wang, W. Zhang, Q. Zhang, and G. Z. Li, "MultiP-SChlo: multi-label protein subchloroplast localization prediction with Chou's pseudo amino acid composition and a novel multi-label classifier," Bioinformatics, vol. 31, no. 16, pp. 2639-45, Aug 152015. 
[78] M. A. M. Hasan, S. Ahmad, and M. K. I. Molla, "Protein subcellular localization prediction using multiple kernel learning based support vector machine," $J$ Molecular BioSystems, vol. 13, no. 4, pp. 785-795, 2017.

[79] T. Goldberg, T. Hamp, and B. Rost, "LocTree2 predicts localization for all domains of life," J Bioinformatics, vol. 28, no. 18, pp. i458-i465, 2012.

[80] Y. Fukasawa, J. Tsuji, S. C. Fu, K. Tomii, P. Horton, and K. Imai, "MitoFates: improved prediction of mitochondrial targeting sequences and their cleavage sites," Mol Cell Proteomics, vol. 14, no. 4, pp. 1113-26, Apr 2015.

[81] C. Savojardo, P. L. Martelli, P. Fariselli, and R. Casadio, "SChloro: directing Viridiplantae proteins to six chloroplastic sub-compartments," Bioinformatics, vol. 33, no. 3, pp. 347-353, Feb 12017.

[82] J. Joyce, "Bayes' theorem," Stanford Encyclopedia of Philosophy, 2003.

[83] J. L. Gardy et al., "PSORTb v.2.0: expanded prediction of bacterial protein subcellular localization and insights gained from comparative proteome analysis," Bioinformatics, vol. 21, no. 5, pp. 617-23, Mar 12005.

[84] N. Y. Yu et al., "PSORTb 3.0: improved protein subcellular localization prediction with refined localization subcategories and predictive capabilities for all prokaryotes," Bioinformatics, vol. 26, no. 13, pp. 1608-15, Jul 12010.

[85] H. Lee, Z. Tu, M. Deng, F. Sun, and T. Chen, "Diffusion kernel-based logistic regression models for protein function prediction," OMICS, vol. 10, no. 1, pp. 4055, Spring 2006.

[86] M. K. Chung, "Introduction to random fields," J arXiv preprint arXiv:.09660, 2020 .

[87] C. Savojardo, P. L. Martelli, P. Fariselli, and R. Casadio, "DeepSig: deep learning improves signal peptide detection in proteins," Bioinformatics, vol. 34, no. 10, pp. 1690-1696, May 152018.

[88] L. Zhu, R. Hofestädt, and M. Ester, "Tissue-Specific Subcellular Localization Prediction Using Multi-Label Markov Random Fields," J IEEE/ACM transactions on computational biology bioinformatics, vol. 16, no. 5, pp. 1471-1482, 2019.

[89] P. J. Thul and C. Lindskog, "The human protein atlas: A spatial map of the human proteome," Protein Sci, vol. 27, no. 1, pp. 233-244, Jan 2018.

[90] N. S. Altman, "An introduction to kernel and nearest-neighbor nonparametric regression," J The American Statistician, vol. 46, no. 3, pp. 175-185, 1992.

[91] P. Horton et al., "WoLF PSORT: protein localization predictor," Nucleic Acids Res, vol. 35, no. Web Server issue, pp. W585-7, Jul 2007. 
[92] H. S. Garapati, G. Male, and K. Mishra, "Predicting subcellular localization of proteins using protein-protein interaction data," J Genomics, vol. 112, no. 3, pp. 2361-2368, 2020.

[93] M. P. Chandra, "On the generalised distance in statistics," in Proceedings of the National Institute of Sciences of India, 1936, vol. 2, no. 1, pp. 49-55.

[94] K.-C. Chou and D. W. Elrod, "Protein subcellular location prediction," J Protein engineering, vol. 12, no. 2, pp. 107-118, 1999.

[95] G. P. Zhou and K. Doctor, "Subcellular location prediction of apoptosis proteins," J Proteins: Structure, Function, Bioinformatics, vol. 50, no. 1, pp. 44-48, 2003.

[96] H. Ding, L. Liu, F.-B. Guo, J. Huang, and H. Lin, "Identify Golgi protein types with modified mahalanobis discriminant algorithm and pseudo amino acid composition," Protein peptide letters, vol. 18, no. 1, pp. 58-63, 2011.

[97] I. Goodfellow, Y. Bengio, A. Courville, and Y. Bengio, Deep learning (no. 2). MIT press Cambridge, 2016.

[98] Y. Bengio, Learning deep architectures for AI. Now Publishers Inc, 2009.

[99] D. E. Rumelhart, G. E. Hinton, and R. J. Williams, "Learning representations by back-propagating errors," J nature, vol. 323, no. 6088, pp. 533-536, 1986.

[100] D. Bahdanau, K. Cho, and Y. Bengio, "Neural machine translation by jointly learning to align and translate," arXiv preprint, 2014.

[101] C. Mooney, Y. H. Wang, and G. Pollastri, "SCLpred: protein subcellular localization prediction by N-to-1 neural networks," Bioinformatics, vol. 27, no. 20, pp. 2812-9, Oct 152011.

[102] X. Wang, Y. Jin, and Q. Zhang, "DeepPred-SubMito: A Novel Submitochondrial Localization Predictor Based on Multi-Channel Convolutional Neural Network and Dataset Balancing Treatment," J International Journal of Molecular Sciences, vol. 21, no. 16, p. 5710, 2020.

[103] J. J. Almagro Armenteros, C. K. Sonderby, S. K. Sonderby, H. Nielsen, and O. Winther, "DeepLoc: prediction of protein subcellular localization using deep learning," Bioinformatics, vol. 33, no. 21, pp. 3387-3395, Nov 12017.

[104] Y. Jiang et al., "MULocDeep: A deep-learning framework for protein subcellular and suborganellar localization prediction with residue-level interpretation," Research Square, 2020.

[105] C. Savojardo, N. Bruciaferri, G. Tartari, P. L. Martelli, and R. Casadio, "DeepMito: accurate prediction of protein submitochondrial localization using convolutional neural networks," Bioinformatics, vol. 36, no. 1, pp. 56-64, 2019. 
[106] C. Savojardo, P. L. Martelli, P. Fariselli, G. Profiti, and R. Casadio, "BUSCA: an integrative web server to predict subcellular localization of proteins," Nucleic Acids Res, vol. 46, no. W1, pp. W459-W466, Jul 22018.

[107] M. Salvatore, P. Warholm, N. Shu, W. Basile, and A. Elofsson, "SubCons: a new ensemble method for improved human subcellular localization predictions,"

Bioinformatics, vol. 33, no. 16, pp. 2464-2470, Aug 152017.

[108] Y. Jiang, D. Wang, and D. Xu, "DeepDom: Predicting protein domain boundary from sequence alone using stacked bidirectional LSTM," Pac Symp Biocomput, vol. 24, pp. 66-75, 2019.

[109] K. D. Tsirigos, C. Peters, N. Shu, L. Kall, and A. Elofsson, "The TOPCONS web server for consensus prediction of membrane protein topology and signal peptides," Nucleic Acids Res, vol. 43, no. W1, pp. W401-7, Jul 12015.

[110] A. Bairoch and R. Apweiler, "The SWISS-PROT protein sequence database and its supplement TrEMBL in 2000," Nucleic acids research, vol. 28, no. 1, pp. 4548, 2000.

[111] W. Li and A. Godzik, "Cd-hit: a fast program for clustering and comparing large sets of protein or nucleotide sequences," Bioinformatics, vol. 22, no. 13, pp. 16581659, 2006.

[112] S. Henikoff and J. G. Henikoff, "Amino acid substitution matrices from protein blocks," Proceedings of the National Academy of Sciences, vol. 89, no. 22, pp. 10915-10919, 1992.

[113] B. Thal, H. P. Braun, and H. Eubel, "Proteomic analysis dissects the impact of nodulation and biological nitrogen fixation on Vicia faba root nodule physiology," Plant molecular biology, vol. 97, no. 3, pp. 233-251, 2018.

[114] B. W. Matthews, "Comparison of the predicted and observed secondary structure of T4 phage lysozyme," Biochimica et Biophysica Acta-Protein Structure, vol. 405, no. 2, pp. 442-451, 1975.

[115] S. Boughorbel, F. Jarray, and M. El Anbari, "Optimal classifier for imbalanced data using Matthews Correlation Coefficient metric," PloS one, vol. 12, no. 6, 2017.

[116] X. Cheng, X. Xiao, and K. C. Chou, "pLoc-mEuk: Predict subcellular localization of multi-label eukaryotic proteins by extracting the key GO information into general PseAAC," Genomics, vol. 110, no. 1, pp. 50-58, Jan 2018.

[117] J. Gorodkin, "Comparing two K-category assignments by a K-category correlation coefficient," Computational biology and chemistry, vol. 28, no. 5-6, pp. 367-374, 2004. 
[118] O. Wagih, "ggseqlogo: a versatile R package for drawing sequence logos," Bioinformatics, vol. 33, no. 22, pp. 3645-3647, 2017.

[119] M. C. Frith, N. F. Saunders, B. Kobe, and T. L. Bailey, "Discovering sequence motifs with arbitrary insertions and deletions," PLoS computational biology, vol. 4, no. 5, p. e1000071, 2008.

[120] C. Dingwall and R. A. Laskey, "Nuclear targeting sequences-a consensus?," Trends in biochemical sciences, vol. 16, pp. 478-481, 1991.

[121] A. Costantino, F. Balzola, and G. Bounous, "Changes in biliary secretory immunoglobulins A in mice fed whey proteins," Minerva dietologica e gastroenterologica, vol. 35, no. 4, pp. 241-245, 1989.

[122] M. I. Lomax, M. D. Welch, B. T. Darras, U. Francke, and L. I. Grossman, "Novel use of a chimpanzee pseudogene for chromosomal mapping of human cytochrome c oxidase subunitIV," Gene, vol. 86, no. 2, pp. 209-216, 1990.

[123] O. Emanuelsson, H. Nielsen, S. Brunak, and G. Von Heijne, "Predicting subcellular localization of proteins based on their N-terminal amino acid sequence," Journal of molecular biology, vol. 300, no. 4, pp. 1005-1016, 2000.

[124] J. Ast, A. C. Stiebler, J. Freitag, and M. Bolker, "Dual targeting of peroxisomal proteins," Front Physiol, vol. 4, p. 297, Oct 182013.

[125] S. Reumann, "Specification of the peroxisome targeting signals type 1 and type 2 of plant peroxisomes by bioinformatics analyses," Plant Physiol, vol. 135, no. 2, pp. 783-800, Jun 2004.

[126] S. Kosugi, M. Hasebe, M. Tomita, and H. Yanagawa, "Nuclear export signal consensus sequences defined using a localization-based yeast selection system," Traffic, vol. 9, no. 12, pp. 2053-62, Dec 2008.

[127] T. E. Kaplinger, V. S. Moore, and W. L. Nusbickel, "Self-documentation for representational state transfer (REST) application programming interface (API)," ed: Google Patents, 2018.

[128] A. L. De Magalhaes, "Modeling representational state transfer application programming interfaces," ed: Google Patents, 2018.

[129] S. E. Calvo and V. K. Mootha, "The mitochondrial proteome and human disease," Annual review of genomics human genetics, vol. 11, pp. 25-44, 2010.

[130] M. Peyro, M. Soheilypour, B. L. Lee, and M. R. Mofrad, "Evolutionarily Conserved Sequence Features Regulate the Formation of the FG Network at the Center of the Nuclear Pore Complex," Sci Rep, vol. 5, p. 15795, Nov 62015. 
[131] D. Ando, M. Colvin, M. Rexach, and A. Gopinathan, "Physical motif clustering within intrinsically disordered nucleoporin sequences reveals universal functional features," PLoS One, vol. 8, no. 9, p. e73831, 2013.

[132] M. N. Chahine and G. N. Pierce, "Therapeutic targeting of nuclear protein import in pathological cell conditions," Pharmacol Rev, vol. 61, no. 3, pp. 358-72, Sep 2009.

[133] X. Zhang et al., "Mutation in nuclear pore component NUP155 leads to atrial fibrillation and early sudden cardiac death," Cell, vol. 135, no. 6, pp. 1017-27, Dec 122008.

[134] L. Basel-Vanagaite et al., "Mutated nup62 causes autosomal recessive infantile bilateral striatal necrosis," Ann Neurol, vol. 60, no. 2, pp. 214-22, Aug 2006.

[135] T. Kiriyama, M. Hirano, H. Asai, M. Ikeda, Y. Furiya, and S. Ueno, "Restoration of nuclear-import failure caused by triple A syndrome and oxidative stress," Biochem Biophys Res Commun, vol. 374, no. 4, pp. 631-4, Oct 32008.

[136] N. Braverman et al., "Human PEX7 encodes the peroxisomal PTS2 receptor and is responsible for rhizomelic chondrodysplasia punctata," Nat Genet, vol. 15, no. 4, pp. 369-76, Apr 1997.

[137] S. Onesti and S. A. MacNeill, "Structure and evolutionary origins of the CMG complex," Chromosoma, vol. 122, no. 1-2, pp. 47-53, Mar 2013.

[138] S. Dalton and L. Whitbread, "Cell cycle-regulated nuclear import and export of Cdc47, a protein essential for initiation of DNA replication in budding yeast," Proc Natl Acad Sci U S A, vol. 92, no. 7, pp. 2514-8, Mar 281995.

[139] M. E. Liku, V. Q. Nguyen, A. W. Rosales, K. Irie, and J. J. Li, "CDK phosphorylation of a novel NLS-NES module distributed between two subunits of the Mcm2-7 complex prevents chromosomal rereplication," Mol Biol Cell, vol. 16 , no. 10 , pp. 5026-39, Oct 2005.

[140] E. H. Armstrong, D. Goswami, P. R. Griffin, N. Noy, and E. A. Ortlund, "Structural basis for ligand regulation of the fatty acid-binding protein 5, peroxisome proliferator-activated receptor beta/delta (FABP5-PPARbeta/delta) signaling pathway," J Biol Chem, vol. 289, no. 21, pp. 14941-54, May 232014.

[141] G. D. Matthews, N. Gur, W. J. Koopman, O. Pines, and L. Vardimon, "Weak mitochondrial targeting sequence determines tissue-specific subcellular localization of glutamine synthetase in liver and brain cells," J Journal of cell science, vol. 123, no. 3, pp. 351-359, 2010.

[142] A. R. Cho et al., "Tissue-specific expression and subcellular localization of ALADIN, the absence of which causes human triple A syndrome," Exp Mol Med, vol. 41, no. 6, pp. 381-6, Jun 302009. 
[143] M. Levadoux, C. Mahon, J. H. Beattie, H. M. Wallace, and J. E. Hesketh, "Nuclear import of metallothionein requires its mRNA to be associated with the perinuclear cytoskeleton," J Biol Chem, vol. 274, no. 49, pp. 34961-6, Dec 3 1999.

[144] N. Gadir, L. Haim-Vilmovsky, J. Kraut-Cohen, and J. E. Gerst, "Localization of mRNAs coding for mitochondrial proteins in the yeast Saccharomyces cerevisiae," RNA, vol. 17, no. 8, pp. 1551-65, Aug 2011.

[145] G. Zipor, L. Haim-Vilmovsky, R. Gelin-Licht, N. Gadir, C. Brocard, and J. E. Gerst, "Localization of mRNAs coding for peroxisomal proteins in the yeast, Saccharomyces cerevisiae," Proc Natl Acad Sci U S A, vol. 106, no. 47, pp. 19848-53, Nov 242009.

[146] C. E. Holt and E. M. Schuman, "The central dogma decentralized: new perspectives on RNA function and local translation in neurons," Neuron, vol. 80, no. 3, pp. 648-57, Oct 302013.

[147] D. Wang et al., "DM3Loc: multi-label mRNA subcellular localization prediction and analysis based on multi-head self-attention mechanism," Nucleic Acids Res, Jan 272021.

[148] A. Vaswani et al., "Attention is all you need," arXiv preprint arXiv:.03762, 2017.

[149] K. Clark, M.-T. Luong, Q. V. Le, and C. D. Manning, "Electra: Pre-training text encoders as discriminators rather than generators," arXiv preprint arXiv:.10555, 2020.

[150] W.-K. Huh et al., "Global analysis of protein localization in budding yeast," Nature, vol. 425, no. 6959, pp. 686-691, 2003.

[151] N. Sabherwal, K. U. Schneider, R. J. Blaschke, A. Marchini, and G. Rappold, "Impairment of SHOX nuclear localization as a cause for Leri-Weill syndrome," $J$ Cell Sci, vol. 117, no. Pt 14, pp. 3041-8, Jun 152004.

[152] A. Hayama, T. Rai, S. Sasaki, and S. Uchida, "Molecular mechanisms of Bartter syndrome caused by mutations in the BSND gene," Histochem Cell Biol, vol. 119, no. 6, pp. 485-93, Jun 2003.

[153] V. Pejaver et al., "Inferring the molecular and phenotypic impact of amino acid variants with MutPred2," vol. 11, no. 1, pp. 1-13, 2020.

[154] J. Thusberg and M. Vihinen, "Bioinformatic analysis of protein structure-function relationships: case study of leukocyte elastase (ELA2) missense mutations,"

Human mutation, vol. 27, no. 12, pp. 1230-1243, 2006. 
[155] J. Thusberg and M. Vihinen, "Pathogenic or not? And if so, then how? Studying the effects of missense mutations using bioinformatics methods," Human mutation, vol. 30, no. 5, pp. 703-714, 2009.

[156] S. Park, J. S. Yang, Y. E. Shin, J. Park, S. K. Jang, and S. Kim, "Protein localization as a principal feature of the etiology and comorbidity of genetic diseases," Mol Syst Biol, vol. 7, p. 494, May 242011.

[157] Z. Liu and J. Hu, "Mislocalization-related disease gene discovery using gene expression based computational protein localization prediction," Methods, vol. 93, pp. 119-127, 2016.

[158] K. Lee et al., "Proteome-wide discovery of mislocated proteins in cancer," Genome Res, vol. 23, no. 8, pp. 1283-94, Aug 2013.

[159] B. A. Lodish H, Zipursky SL, et al., Molecular Cell Biology. W. H. Freeman; 4th edition, 2000.

[160] J. Denecke, R. De Rycke, and J. Botterman, "Plant and mammalian sorting signals for protein retention in the endoplasmic reticulum contain a conserved epitope," EMBO J, vol. 11, no. 6, pp. 2345-55, Jun 1992.

[161] V. Gomord, L. A. Denmat, A. C. Fitchette-Laine, B. Satiat-Jeunemaitre, C. Hawes, and L. Faye, "The C-terminal HDEL sequence is sufficient for retention of secretory proteins in the endoplasmic reticulum (ER) but promotes vacuolar targeting of proteins that escape the ER," Plant J, vol. 11, no. 2, pp. 313-25, Feb 1997.

[162] M. Stornaiuolo et al., "KDEL and KKXX retrieval signals appended to the same reporter protein determine different trafficking between endoplasmic reticulum, intermediate compartment, and Golgi complex," Mol Biol Cell, vol. 14, no. 3, pp. 889-902, Mar 2003. 


\section{VITA}

Yuexu Jiang was born in Changchun, China. He is currently a PhD candidate in EECS Department at the University of Missouri, Columbia (MU), MO 65211, USA. He got BS in Electronic Information Science and Technology at Jilin University, Changchun, China in 2010 and MA in Software Engineering at Jilin University, Changchun, China in 2012. He studied in Dr. Dong Xu's lab at MU as a visiting scholar in 2015 and started the $\mathrm{PhD}$ program in EECS Department at MU in 2016. He got PhD in Bioinformatics at Jilin University, Changchun, China in 2018. During his PhD program in MU, he published 15

papers ( 4 as the first author), and 4 more peer-review papers are in progress ( 3 as the first or co-first author). His research interests include machine learning, deep learning, bioinformatics, and system biology. He will stay at MU as a postdoc jointly supported by Dr. Dong Xu and Dr. Trupti Joshi. 